BULLETIN (New Series) OF THE

AMERICAN MATHEMATICAL SOCIETY

Volume 42, Number 3, Pages 291-363

S 0273-0979(05)01059-1

Article electronically published on April 18, 2005

\title{
FROM ATOMS TO CRYSTALS: A MATHEMATICAL JOURNEY
}

\author{
CLAUDE LE BRIS AND PIERRE-LOUIS LIONS
}

\begin{abstract}
We present an overview of some works on the models of computational quantum chemistry. We examine issues such as the existence of ground states (both for the electronic structure and the configuration of nuclei), the foundations of the models of the crystalline phase, and the macroscopic limits. We emphasize the connections between the physical modelling, the numerical concerns and the mathematical analysis of the problems.
\end{abstract}

\section{OutLine}

This article presents an expository review of some collective work by a group of researchers (X. Blanc, E. Cancès, I. Catto, M. Esteban, E. Séré) to which we belong on the mathematical aspects of the models used for the simulation of matter at the microscopic scale. The relation of these models to models for the continuum description of matter at the macroscopic scale will also be examined.

The mathematical material reported on here covers almost two decades, starting in the late 1980s with our contribution to a general endeavour (mainly initiated by E.H. Lieb, B. Simon, W. Thirring) to put the models of quantum chemistry on a sound mathematical basis (see Sections 3 to 5 . It continues in the 1990s with our work on the thermodynamic limit of molecular models in order to establish and/or justify models for the crystal phase (see Section 6). The most recent step (in fact hardly addressed here, only outlined in Section [7, and postponed until future publications) is the definition of the energy of general infinite microscopic sets of particles and the passage to the macroscale with the tentative definition of the density of mechanical energy on the basis of models at the atomistic scale.

The program described above is ambitious. However, we are in a position where on the one hand some building blocks of the whole program are indeed known from the mathematical standpoint for most of the models used in practice, and on the other hand where we are able to accomplish all the steps for some models, rather academic in nature but nevertheless illustrative and of some physical relevance. Therefore, we believe that this is the proper time for such a survey article. Enough convincing arguments are available to prove that it is indeed possible to analyze models of microscopic matter and to determine the macroscopic limit, on a mathematical ground, provided one restricts one's ambition. A large body

Received by the editors November 20, 2004.

2000 Mathematics Subject Classification. Primary 35Bxx, 35Jxx, 35Pxx, 49Kxx, 81Q05, 81Q10, 82Bxx.

This article is an extended version by the two authors of notes based upon a series of lectures given by PLL at Collège de France during the fall semester of the academic year 2003/04.

(C)2005 American Mathematical Society Reverts to public domain 28 years from publication 
of mathematical literature confirms this. It is now time to give to the interested mathematician a user's guide to such an extensive literature.

On the other hand, we must give an unbiased view of the enterprise. Some building blocks are missing. There remain many unresolved issues from a purely mathematical viewpoint. We shall therefore indicate some open problems that are of mathematical and, within the limits of our competence in the field, physical interest. One of our hopes in writing such a survey is that it will motivate further efforts by mathematicians so that the remaining steps could be covered within a reasonable future.

For the sake of brevity, we have chosen to outline the main results obtained over the years in the following fashion. We will only state the results in their more illustrative form, omitting on purpose possible extensions and technicalities. In the same spirit, we will only sketch very briefly the proofs in the simplest cases and give some hints on how to extend the results. One of our main goals is to present the mathematical tools without entering into the details and the technicalities of proofs, and also to emphasize the articulations between the results. To put it otherwise, we have collected here what is not in the articles: there the detailed, sometimes tedious, proofs can be found, as well as the most general statement of the results. The price to pay for brevity will sometimes be vagueness: we will take some liberty with rigor and refer the reader to the bibliography herein for detailed arguments and statements.

We will also take advantage of this survey, definitely more on a mathematical ground, to give a flavour of the challenges from the numerical viewpoint. As testified by the Nobel Prize awarded in 1998 to Walter Kohn and John Pople (see the Nobel Lecture [136]), computational chemistry has indeed gained full recognition in the world of chemistry, a domain traditionally more experimentally-oriented. Therefore, we will incidentally allow ourselves some quick incursion into the numerical issues. In the same spirit as for our theoretical concerns, we will also identify some numerical bottlenecks and some approaches to overcome them (see mainly Section 3.3).

Let us conclude this introduction by mentioning some general references. For the general background of quantum mechanics, we refer to the treatises [148, 171. that are both biased towards molecular quantum chemistry. On the other hand, some basic mathematical material related to the field of mathematical physics can be found in the monographs $31,32,74,123,185,187,196,201$. In addition to this reference list, we point out some of our own works: the treatises [82, 141] (and in particular [48] therein) and the survey article [146], where more details are given on the modelling and the numerical background.

The bibliography enclosed at the end of this article includes more references than those cited in the body of the text.

\section{The Schrödinger EQuation and the computational Bottleneck}

Quantum chemistry tries to understand and simulate the properties of matter via the modelling of its behaviour at the microscopic scale. The motivation is that many macroscopic properties originate from elementary processes which take place at the atomic scale. Let us mention for instance the fact that the elastic constants of a perfect crystal or the color of a chemical compound can be evaluated by atomic scale calculations. It is thus reasonable to start from such a microscopic description. 
On the other hand, some other macroscopic phenomena proceed from bulk effects and will not be satisfactorily captured solely by a microscopic model.

At the microscopic scale, and in a stationary picture, the mathematical description is provided by the Schrödinger equation

$$
H \Psi=E \Psi,
$$

where $H$ denotes the quantum Hamiltonian of the molecular system under consideration (a self-adjoint operator on some Hilbert space $\mathcal{H}$ ) and $\Psi$ is the wavefunction of the system (unit vector in $\mathcal{H}$ ). The scalar $E$ denotes the energy.

This model can be considered as a universal model (except in a few special cases, notably those involving relativistic phenomena - models for such situations will be briefly addressed in Section 3.1.6 below - or nuclear reactions) for it contains all the physical information of the system, does not involve any empirical parameter and enjoys remarkable predictive capabilities, as confirmed by comparisons with experiment.

In addition, it is natural to focus on the determination of the solution to (2.1) for the lowest possible energy $E$, which amounts to determining the ground state (i.e. state of minimal energy) of the system under consideration. In the natural environment indeed, chemical and physical systems are usually (not always!) found in their most stable state. Likewise any chemical system $A$ reacts, spontaneously or with a compound $X$, to give products $B, C, \ldots$ according to a chemical reaction if it is accompanied by a variation of energy stabilizing the whole system. The above thermodynamic consideration does not suffice however to explain all the observations: other states than the most stable one can be found and reactions that are thermodynamically possible may happen after billions of years. But the determination of the ground state and of the ground-state energy remains the "must" of the computational chemistry, a preliminary calculation needed before turning to other questions.

2.1. The basic modelling. In principle, all the components of the system under study need to be accounted for in the definition of $H$ and $\mathcal{H}$. In the present context of chemistry, we may consider that the elementary objects the molecular system is composed of are electrons and nuclei. There is no need to account in detail for the substructure of the nuclei. Let us henceforth denote by $M$ and $N$ the total number of nuclei and electrons in the system, respectively.

In most of the settings of chemistry, it is reasonable to consider the nuclei as classical objects, and as point-like particles clamped at positions $\left(\bar{x}_{1}, \cdots, \bar{x}_{M}\right)$. This stems from the so-called Born-Oppenheimer approximation.

In view of this approximation, problem (2.1) can be rewritten in the form

$$
H_{e}^{\left(\bar{x}_{1}, \cdots, \bar{x}_{M}\right)} \psi_{e}=E_{e} \psi_{e},
$$

where the electronic Hamiltonian $H_{e}^{\left(\bar{x}_{1}, \cdots, \bar{x}_{M}\right)}$ is parameterized by the positions of the nuclei and reads

$$
H_{e}^{\left(\bar{x}_{1}, \cdots, \bar{x}_{M}\right)}=-\sum_{i=1}^{N} \frac{1}{2} \Delta_{x_{i}}-\sum_{i=1}^{N} \sum_{k=1}^{M} \frac{z_{k}}{\left|x_{i}-\bar{x}_{k}\right|}+\sum_{1 \leq i<j \leq N} \frac{1}{\left|x_{i}-x_{j}\right|} .
$$

In order to write $H_{e}^{\left(\bar{x}_{1}, \cdots, \bar{x}_{M}\right)}$, we have chosen the atomic unit system, commonly used in quantum chemistry, that we shall adopt throughout this article. In this 
system,

$$
m_{e}=1, \quad e=1, \quad \hbar=1, \quad \frac{1}{4 \pi \epsilon_{0}}=1,
$$

where $m_{e}, e, \hbar, \epsilon_{0}$ respectively denote the electron mass, the elementary charge, the reduced Planck constant, and the dielectric permittivity of a vacuum. The charge of the $k$-th nucleus is denoted by $z_{k}$. Beyond the simplicity of this system, which motivates its use both in mathematics and in chemistry, one must keep in mind the formidably tiny space and time scales that are involved. The typical size of the electronic cloud of an isolated atom is the Angström ( $10^{-10}$ meter). The size of the nucleus embedded therein is $10^{-15}$ meter. The weight of an atom is of the order of $10^{-26} \mathrm{~kg}$. Regarding the time scale, the typical vibration period of a molecular bond is the femtosecond $\left(10^{-15}\right.$ second), and the characteristic relaxation time for an electron is $10^{-18}$ second. Consequently, quantum chemistry calculations concern very short time behaviors of very small and very light systems.

The above Hamiltonian (2.3) can be deduced from the one in the classical mechanics framework,

$$
\begin{aligned}
H_{c l}= & \sum_{i=1}^{N} \frac{p_{x_{i}}^{2}}{2}-\sum_{i=1}^{N} \sum_{k=1}^{M} \frac{z_{k}}{\left|x_{i}-\bar{x}_{k}\right|} \\
& +\sum_{1 \leq i<j \leq N} \frac{1}{\left|x_{i}-x_{j}\right|},
\end{aligned}
$$

by replacing the $x$ component by the operator consisting in a multiplication by $x$, and the momentum $p_{x}$ along the $x$ component by the operator $-i \nabla_{x}$. The meaning of each term in (2.3) is then clear: the first term corresponds to the kinetic energy of the electrons; the latter two terms model the electrostatic energy between the particles, between nuclei and electrons first, between electrons secondly.

The second term of (2.3), namely the attraction of the nuclei on the electrons, will henceforth be denoted by

$$
V_{0}\left(x_{1}, \ldots, x_{N}\right)=\sum_{i=1}^{N} V\left(x_{i}\right)=-\sum_{i=1}^{N}\left(\sum_{k=1}^{M} \frac{z_{k}}{\left|\cdot-\bar{x}_{k}\right|}\right)\left(x_{i}\right) .
$$

Regarding the variational space $\mathcal{H}_{e}$ in which the wavefunction $\psi_{e}$ varies, we need to make a few remarks. According to the basics of quantum mechanics, it is some subspace of a space $L^{2}\left(\mathbb{R}^{d}\right)$, for some dimension $d$ related to the total number of electrons in the system. We note that because of the Pauli principle, the electronic wavefunction is by construction antisymmetric with respect to the exchange of the coordinates of both space and spin of two electrons. Therefore the subspace under consideration will consist of antisymmetric functions. We also note that the Hamiltonian $H_{e}^{\left(\bar{x}_{1}, \cdots, \bar{x}_{M}\right)}$ does not act on the spin variable, and we therefore omit the spin variable in all that follows. The dimension $d$ is therefore $d=3 N$. The reader should only keep in mind that all the models we shall introduce and the arguments we shall make can be slightly modified in order to account for the spin. This is sometimes done at the price of some substantial tedious details but never is a conceptual difficulty (basically because our main concern deals with the compactness of all the variational problems at hand, and that the spin variable varies in a finite thus compact set). Likewise, we remark that $H_{e}^{\left(\bar{x}_{1}, \cdots, \bar{x}_{M}\right)}$ is a real operator, and thus it suffices to consider real-valued wavefunctions to entirely determine the ground 
state. In addition, it is also clear from the form of the Hamiltonian $H_{e}^{\left(\bar{x}_{1}, \cdots, \bar{x}_{M}\right)}$ that, if we want to only consider states of finite energy, we need to impose the $L^{2}$ integrability of the first derivative of the wavefunctions with respect to the space variables. This is the necessary and sufficient condition to give a meaning to the kinetic energy terms. In view of standard results in functional analysis, this also allows one to properly define the electrostatic Coulombian interaction term. Therefore, the variational space for (2.2) is set to the antisymmetrized tensor product:

$$
\mathcal{H}_{e}=\bigwedge_{i=1}^{N} H^{1}\left(\mathbb{R}^{3}\right) .
$$

Of course, an alternative viewpoint to the one above is to remark that (2.2) is the Euler-Lagrange equation of the minimization problem

$$
U\left(\bar{x}_{1}, \cdots, \bar{x}_{M}\right)=\inf \left\{\left\langle\psi_{e}, H_{e}^{\left(\bar{x}_{1}, \cdots, \bar{x}_{M}\right)} \psi_{e}\right\rangle, \quad \psi_{e} \in \mathcal{H}_{e}, \quad\left\|\psi_{e}\right\|_{L^{2}}=1\right\} .
$$

The energy of the complete system consisting of the classical nuclei and the electrons is then recovered by setting

$$
W\left(\bar{x}_{1}, \cdots, \bar{x}_{M}\right)=U\left(\bar{x}_{1}, \cdots, \bar{x}_{M}\right)+\sum_{1 \leq k<l \leq M} \frac{z_{k} z_{l}}{\left|\bar{x}_{k}-\bar{x}_{l}\right|} .
$$

2.2. Direct approaches at the Schrödinger level. Even in the "simplified" form (2.7), the minimization problem, however enjoying good mathematical properties since it consists of the minimization of a quadratic form (see [105] or [128] for a review), is untractable from the practical viewpoint again because of the dimension of the variational space. Indeed, already for $N$ of the order of 10 (which is the case for the water molecule), the approximation of $L^{2}\left(\mathbb{R}^{3 N}\right)$, or more precisely that of its subspace $\bigwedge_{i=1}^{N} H^{1}\left(\mathbb{R}^{3}\right)$, is an overwhelming task practically, and thus the direct attack of problem (2.7) is not possible. Such a direct attack is thus currently restricted to systems of very small size.

For most, if not all, systems, additional approximations are thus necessary in order to allow for computations. In one way or another, all of these simplifications aim at reducing the dimensionality of the problem. We will introduce them in the next section.

\section{Remark 2.1. On bielectronic integrals.}

In addition to this dimensionality problem, a second difficulty comes from the fact that the computation of $\left\langle\psi_{e}, H_{e}^{\left(\bar{x}_{1}, \cdots, \bar{x}_{M}\right)} \psi_{e}\right\rangle$ for an arbitrary $\psi_{e}$ involves integrals of the form

$$
\int_{\mathbf{R}^{3 N}} \frac{1}{\left|x_{i}-x_{j}\right|}\left|\psi_{e}\right|^{2} d x_{1} \ldots d x_{N}
$$

due to the presence of the electrostatic repulsion between electrons in (2.3). The calculation of these integrals (called in chemistry the bielectronic integrals integrals, for they involve the positions of two electrons simultaneously) is a substantial practical difficulty that is treated today by dedicated methods. Stochastic-based methods as well as clever deterministic approaches can be employed.

Remark 2.2. On the exact wavefunction. With a view to designing efficient discretization schemes for further approximation of (2.7), it is useful to keep track of the mathematical properties of the exact wavefunction $\psi_{e}$ minimizing (2.7), or 
equivalently providing a solution to (2.2). It is indeed easily seen that $\psi_{e}$ has a cusp at each point nucleus, the shape of which is related to the charge of the nucleus, and that the density $\rho$ is an exponentially decreasing function at infinity. These two properties have a great impact on the modelling because it is important to have approximations that mimick these behaviours. Likewise, they impact on the choice of the discretization space, as the basis functions adopted indeed should reproduce well this behaviour at short and long distances, for the sake of efficiency. We refer to 110, 111, 112, 127, 204, 205, 206 for works related to these issues of the regularity of the wavefunction and of the density.

Before we turn to the approximations of (2.7), and with a view to enlarging the practical applicability of problem (2.7), we would like to mention the stochastic approach, that is, one possible way to treat (2.7) for a number $N$ of electrons that are not necessarily small.

It is already known in the chemistry literature (see e.g. [147]) that Monte-Carlolike methods can be the methods of choice for treating problem (2.7). The basic observation that underlies the approach is the following. In order to compute (2.7), the following time-dependent parabolic equation can be considered:

$$
\frac{\partial \psi_{e}}{\partial t}+H_{e} \psi_{e}=0
$$

As $t$ goes to infinity,

$$
\frac{1}{t} \log \left|\psi_{e}(t, x)\right|=\frac{1}{t} \log \left|e^{-t H_{e}} \psi_{e}(0, x)\right| \longrightarrow \quad U \quad \text { (defined by (2.7)). }
$$

Now, we know by the Feynmann-Kac formula that $\psi_{e}(t, x)$ reads as the following expectation value:

$$
\psi_{e}(t, x)=\mathbb{E}\left(\psi_{e}^{0}\left(x+W_{t}\right) \exp \left(-\int_{0}^{t}\left(V_{0}+V_{1}\right)\left(x+W_{s}\right) d s\right)\right),
$$

where $\psi_{e}^{0}$ is an initial datum for (2.9), $V_{0}$ is defined by 2.5), $V_{1}$ denotes the last term of (2.3), and $W_{t}$ denotes a $3 N$-dimensional Brownian motion.

Therefore, the calculation of $U$ amounts to determining the long time limit (2.10), and thus to evaluating by empirical means the expectation value (2.11). In practice, the main two difficulties are the need to ensure that $\psi_{e}$ is an antisymmetric function, and the need to generate in the stochastic simulation only points that will significantly contribute to the expectation value (2.11). In this direction, recent developments in other traditional fields of applications for stochastic methods such as financial mathematics could constitute a promising mathematical approach for chemistry (see [169] for an outline of the strategy and [57] for a related and recent mathematical study).

2.3. Approximations. The most commonly used approximations to the minimization problem (2.7) can schematically be classified into two main classes:

- wavefunction methods aim at finding an approximation of the ground-state electronic wavefunction, i.e. of the minimizer of (2.7). The variational space $\mathcal{H}_{e}$ is reduced but the "exact" form of the energy $\left\langle\psi_{e}, H_{e}^{\left(\bar{x}_{1}, \cdots, \bar{x}_{M}\right)} \psi_{e}\right\rangle$ is kept. The Hartree-Fock approximation, introduced by Hartree and improved by Fock in the late 1920s, is the most famous example of such a method. Wavefunction methods are used preferably by chemists who are interested in the precise simulations of systems of small size, when computational 
time is not an issue. We refer to the treatises [126, 199] in the chemistry literature.

- density functional methods are issued from the Density Functional Theory, an ancestor of which is the Thomas-Fermi approximation, well known by mathematicians. They are based on a reformulation of problem (2.7) in such a way that the main variable is the electronic density

$$
\rho(x)=N \int_{\mathbf{R}^{3(N-1)}}\left|\psi_{e}\left(x, x_{2}, \cdots, x_{N}\right)\right|^{2} d x_{2} \cdots d x_{N}
$$

(i.e. a scalar field on $\mathbb{R}^{3}$ ) rather than the wavefunction (i.e. a scalar field on $\mathbb{R}^{3 N}$ ) as in the original problem (2.7). This is why these methods are widely used by those chemists who are interested in large molecular systems (e.g. biological systems) as well as by most solid-state physicists. The fact that various parameters or even the very form of some terms of the energy functional need to be arbitrarily chosen or tuned for these methods makes the method particularly efficient for some situations but is sometimes seen as a lack of rigour by chemists.

The approximations outlined above and that we will now introduce are called $a b$ initio approximations. They all consist in a simplified but still quantum description of the electronic structure around the nuclei. One may nevertheless follow an alternative approach. The so-called molecular mechanics models consist of replacing (2.8) by a fully classical energy of the type

$$
U^{\text {classic }}\left(\bar{x}_{1}, \cdots, \bar{x}_{M}\right)+\sum_{1 \leq k<l \leq M} \frac{z_{k} z_{l}}{\left|\bar{x}_{k}-\bar{x}_{l}\right|} .
$$

This allows for the practical treatment of systems of millions of nuclei.

Remark 2.3. On the use of ab initio methods. Although ab initio methods are restricted to small systems (both in terms of space and in terms of time), it is to be mentioned that they have a twofold interest:

- they can indeed be used to simulate very precisely small systems, which has a great interest per se

- and they can also be used in a coupled simulation of a far larger system, either by using them in a preliminary step to compute and fit interaction potentials between atoms (next inserted in formulae of the type (2.12)), or by coupling two levels of approximation, namely the ab initio one and the empirical one.

\section{Finding the EleCtronic Ground StATE}

\subsection{Hartree-Fock type models.}

3.1.1. Physical modelling. The Hartree-Fock approximation, abbreviated by HF, consists of restricting in the variational problem (2.7) the variational space $\mathcal{H}_{e}$ to that of functions of the variables $\left(x_{1}, \ldots, x_{N}\right) \in \mathbb{R}^{3 N}$ that can be written as a single determinant (i.e. an antisymmetrized product) of $N$ functions defined on $\mathbb{R}^{3}$. Recall that, in its entire generality, an arbitrary element of $\mathcal{H}_{e}$ is only a converging infinite sum of such determinants. The Hartree-Fock approximation is therefore defined as

$$
U^{H F}\left(\bar{x}_{1}, \cdots, \bar{x}_{M}\right)=\inf \left\{\left\langle\psi_{e}, H_{e} \psi_{e}\right\rangle, \quad \psi_{e} \in \mathcal{S}_{N}\right\},
$$


with

$\mathcal{S}_{N}=\left\{\psi_{e}=\frac{1}{\sqrt{N !}} \operatorname{det}\left(\phi_{i}\left(x_{j}\right)\right), \quad \phi_{i} \in H^{1}\left(\mathbb{R}^{3}\right), \quad \int_{\mathbb{R}^{3}} \phi_{i} \phi_{j}=\delta_{i j}, \quad 1 \leq i, j \leq N\right\}$.

In order to make the form of the wavefunction more explicit for the reader, we expand it as follows:

$$
\psi_{e}\left(x_{1}, \cdots, x_{N}\right)=\frac{1}{\sqrt{N !}} \operatorname{det}\left(\phi_{i}\left(x_{j}\right)\right)=\frac{1}{\sqrt{N !}}\left|\begin{array}{ccc}
\phi_{1}\left(x_{1}\right) & \cdots & \phi_{1}\left(x_{N}\right) \\
\cdot & & \cdot \\
\cdot & & \cdot \\
\cdot & \\
\phi_{N}\left(x_{1}\right) & \cdots & \phi_{N}\left(x_{N}\right)
\end{array}\right|
$$

In the language of quantum chemistry, a function of the form (3.3) is called a Slater determinant, and the $\phi_{i}$ are called molecular orbitals.

Apart from purely technical antisymmetry considerations, the Hartree-Fock approximation heuristically consists of writing that the probability density $|\psi|^{2}\left(x_{1}, \ldots, x_{N}\right)$ of finding the $N$ electrons at positions $\left(x_{1}, \ldots, x_{N}\right)$ can be written as the product $\left|\phi_{1}\right|^{2}\left(x_{1}\right) \ldots\left|\phi_{N}\right|^{2}\left(x_{N}\right)$. This amounts to considering the positions of the electrons as independent variables. This simplification causes a loss of correlation between the positions of the electrons and is responsible for some error in the result obtained. Indeed, restricting the minimization to some specific forms of functions in (3.1) provides only an upper bound of the energy (2.7). On the other hand, the fact that it is an upper bound and not only an approximation of the exact energy is of course a substantial practical advantage of the method, in comparison to other, non-variational approximations such as those coming from the density functional theory (see Section 3.1.5 and below).

Let us now write the Hartree-Fock approximation in a more explicit fashion. We denote

$$
\tau\left(x, x^{\prime}\right)=\sum_{i=1}^{N} \phi_{i}(x) \phi_{i}\left(x^{\prime}\right)
$$

and

$$
\rho(x)=\sum_{i=1}^{N}\left|\phi_{i}(x)\right|^{2}
$$

The functions $\tau$ and $\rho$ are respectively called the density matrix and the density associated to the state $\psi_{e}$. The function $\tau$ is in fact a special occurrence for a Slater determinant $\psi_{e}$ of the general formula

$$
\gamma_{1}\left(x, x^{\prime}\right)=\int_{\mathbb{R}^{3(N-1)}} \psi_{e}\left(x, x_{2}, x_{3}, \ldots, x_{N}\right) \psi_{e}\left(x^{\prime}, x_{2}, x_{3}, \ldots, x_{N}\right) d x_{2} \cdots d x_{N}
$$

which defines in the entire generality the first-order reduced density matrix calculated from the state $\psi_{e}$ (objects of this type will be manipulated in Remark 3.13 below). The density $\rho$ is then deduced from $\tau$ by taking the trace. 
This allows us to compute

$$
\begin{aligned}
E^{H F}\left(\phi_{1}, \ldots, \phi_{N}\right)=\left\langle\psi_{e}, H_{e} \psi_{e}\right\rangle= & \sum_{i=1}^{N} \frac{1}{2} \int_{\mathbb{R}^{3}}\left|\nabla \phi_{i}\right|^{2}+\int_{\mathbb{R}^{3}} \rho V \\
& +\frac{1}{2} \int_{\mathbb{R}^{3}} \int_{\mathbb{R}^{3}} \frac{\rho(x) \rho\left(x^{\prime}\right)}{\left|x-x^{\prime}\right|} d x d x^{\prime} \\
& -\frac{1}{2} \int_{\mathbb{R}^{3}} \int_{\mathbb{R}^{3}} \frac{\left|\tau\left(x, x^{\prime}\right)\right|^{2}}{\left|x-x^{\prime}\right|} d x d x^{\prime} .
\end{aligned}
$$

In the sequel to this section, we fix the positions $\left(\bar{x}_{1}, \cdots, \bar{x}_{M}\right)$ of the nuclei, fix the number $N$ of electrons, and now denote $I_{N}^{H F}=U^{H F}\left(\bar{x}_{1}, \cdots, \bar{x}_{M}\right)$; i.e.

$$
\begin{aligned}
I_{N}^{H F}=\inf \left\{\sum_{i=1}^{N} \frac{1}{2} \int_{\mathbb{R}^{3}}\left|\nabla \phi_{i}\right|^{2}+\int_{\mathbf{R}^{3}} \rho V+\frac{1}{2} \int_{\mathbb{R}^{3}} \int_{\mathbf{R}^{3}} \frac{\rho(x) \rho\left(x^{\prime}\right)}{\left|x-x^{\prime}\right|} d x d x^{\prime}\right. \\
-\frac{1}{2} \int_{\mathbf{R}^{3}} \int_{\mathbf{R}^{3}} \frac{\left|\tau\left(x, x^{\prime}\right)\right|^{2}}{\left|x-x^{\prime}\right|} d x d x^{\prime}, \\
\left.\phi_{i} \in H^{1}\left(\mathbb{R}^{3}\right), \quad \int_{\mathbb{R}^{3}} \phi_{i} \phi_{j}=\delta_{i j}, \quad 1 \leq i, j \leq N\right\},
\end{aligned}
$$

where we recall that

$$
V(x)=-\sum_{k=1}^{M} \frac{z_{k}}{\left|x-\bar{x}_{k}\right|} .
$$

The above spinless Hartree-Fock model (3.7) has been extensively studied by mathematicians.

Remark 3.1. In the mathematical literature, the factor $\frac{1}{2}$ in front of the kinetic energy is often omitted. It is of course only a matter of units and does not change at all the analysis nor the conditions (algebraic or not) we will find for the existence of minimizers.

3.1.2. Existence of a ground state: generalities. In view of its importance for computational issues, we focus on the very first question that can be asked about a minimization problem such as (3.7): does there exist a minimizer (i.e. a ground state in terms of chemistry), and what can be said about it?

Very briefly, let us outline the mathematical difficulty. Problem (3.7), along with all the minimization problems we shall encounter in the present section, belongs to a class of problems that we formulate somewhat vaguely in the form

$$
I_{N}=\inf \left\{E(\phi), \quad \int_{\mathbf{R}^{N}}|\phi|^{2}=N\right\}
$$

that are set on the whole space $\mathbb{R}^{N}$ and that involve energy functionals that contain gradient norms; the loss of compactness in a minimizing sequence may occur, neither by oscillation nor concentration, but by escape at infinity. This phenomenon is interpreted in terms of chemistry by ionization, or in other words by the loss of an electron. For this reason, such problems are often called locally compact variational problems. Indeed, the main difficulty does not come from the energy functional. In most cases (with the notable exception of the Thomas-Fermi-Dirac-von Weizsäcker model that we will deal with in Section 4.5), the energy functional is weakly lower semicontinuous for the $H^{1}$ topology. In the majority of these cases, the weakly 
lower semicontinuity in turn originates from some convexity of the functional. The main difficulty rather comes from the constraint. In other words, a sequence such that

$$
\left\{\begin{array}{l}
\int_{\mathbb{R}^{N}}\left|\phi^{n}\right|^{2}=N, \\
\lim _{n \longrightarrow+\infty} E\left(\phi^{n}\right)=I_{N}
\end{array}\right.
$$

may converge to some $\phi^{\infty}$ that in general has the right energy $E\left(\phi^{\infty}\right)=I_{N}$ but that might have an $L^{2}$ norm $\int_{\mathbb{R}^{N}}\left|\phi^{\infty}\right|^{2}$ strictly inferior to $N$. In order to ensure that no such phenomenon occurs, it is natural that spectral theory should play a role at some point, exactly as it does in the mathematical study of the simple quadratic case

$$
I=\inf \left\{\langle(-\Delta+W) \phi, \phi\rangle, \phi \in H^{1}\left(\mathbb{R}^{3}\right), \int|\phi|^{2}=1\right\} .
$$

In the nonlinear case at hand, the Schrödinger operator $-\Delta+W$ (in fact depending on the variable $\phi$ ) is the first-order derivative of the energy at a tentative minimizer. The essential feature is to know whether the potential $W$ is sufficiently negative (i.e. attractive) at finite distance, and sufficiently positive at infinity (i.e. prevents diffusion), so that a minimizer exists, a fact which is interpreted in physics by saying that the Hamiltonian has at least a bound state below the states of diffusion, i.e. the continuous spectrum. This property has then to be checked by careful inspection of each case at hand.

Let us now turn more precisely to the HF case.

3.1.3. Existence of a ground state: the HF case. The main result is the following.

Theorem 3.2. 155, 166] Existence of a minimizer for the HF model

- (i) Any minimizing sequence of (3.7) is relatively compact in $\left(H^{1}\left(\mathbb{R}^{3}\right)\right)^{N}$ if and only if the following condition holds:

$$
I_{N}^{H F}<I_{N-1}^{H F} .
$$

- (ii) Condition (3.10) holds as soon as the total nuclear charge satisfies

$$
Z=\sum_{k=1}^{M} z_{k}>N-1
$$

Some immediate comments are in order, first about the condition (3.11).

Note first that when the $z_{k}$ are integers, which is the physically relevant case (recall we work with atomic units), condition (3.11) also reads $Z \geq N$ and thus (ii) states the existence of a ground state for neutral and positively charged systems (i.e. molecules and cationic ions) in the HF model.

The mathematical condition (3.11) translates the physical property that the nuclei should be sufficiently positively charged in order to be able to bind the $N$ electrons at their vicinity, which is quite a natural assumption that can be understood on the basis of a simple electrostatic balance: when $Z$ is not large enough the $N$ electrons are left free to repel each other and may escape. Such a phenomenon can be formalized and illustrated by the following elementary argument on a simple model of a molecular system consisting of classical (as opposed to quantum) but 
smeared out particles. Consider a set of $M$ nuclei, of (integer) charge $z_{1}, \ldots, z_{M}$, located at points $\bar{x}_{1}, \ldots, \bar{x}_{M}$, respectively. Next, add to this system a set of $N$ electrons, located at the points $x_{1}, \ldots, x_{N}$. To avoid a collapse due to the singularity of the Coulomb potential, it is necessary to smear out the nuclei and the electrons, assuming that their shape is given by a smooth nonnegative function $m$ (such that $\left.\int m=1\right)$ centered at the points $\bar{x}_{k}(1 \leq k \leq M)$ and $x_{i}(1 \leq i \leq N)$ respectively. The density of the nuclei and that of the electrons thus read $\bar{\rho}(x)=\sum_{k=1}^{M} z_{k} m\left(x-\bar{x}_{k}\right)$ and $\rho(x)=\sum_{j=1}^{N} m\left(x-x_{j}\right)$, respectively. Let us introduce the notation

$$
D(f, g)=\int_{\mathbb{R}^{3}} \int_{\mathbf{R}^{3}} \frac{f(x) g(y)}{|x-y|} d x d y,
$$

whenever it makes sense. Then the minimization problem (analogous to the quantum problem (2.7)) to be solved in order to determine the electronic ground state reads

$$
\begin{aligned}
& I_{N}^{\text {classic }}=\inf \{-\sum_{1 \leq k \leq M} \sum_{1 \leq j \leq N} z_{k} D\left(m\left(x-\bar{x}_{k}\right), m\left(x-x_{i}\right)\right) \\
&+\frac{1}{2} \sum_{1 \leq i \neq j \leq N} D\left(m\left(x-x_{i}\right), m\left(x-x_{j}\right)\right) ; \\
&\left.\left(x_{1}, \ldots, x_{N}\right) \in \mathbb{R}^{3 N}\right\}
\end{aligned}
$$

and can be reformulated as

$$
I_{N}^{\text {classic }}=J_{N}^{\text {classic }}-\frac{1}{2} D(\bar{\rho}, \bar{\rho})-\frac{N}{2} D(m, m),
$$

where

$$
J_{N}^{\text {classic }}=\inf \left\{\frac{1}{2} D(\bar{\rho}-\rho, \bar{\rho}-\rho) ; \quad \rho(x)=\sum_{j=1}^{N} m\left(x-x_{j}\right) ; \quad\left(x_{1}, \ldots, x_{N}\right) \in \mathbb{R}^{3 N}\right\} .
$$

Then, the analysis is straightforward and follows from the following simple observation: $D(f, f) \geq 0$ for all $f$ and vanishes if and only if $f \equiv 0$. When $N \leq Z$ (i.e. $N-1<Z$ ), the infimum (3.15), thus (3.13), is attained when the $x_{i}(1 \leq i \leq N)$ are exactly some of the $\bar{x}_{k}(1 \leq k \leq M)$. More precisely, for a minimizer a number of electrons equal to the charge $z_{k}$ is set at the location $x_{i}=\bar{x}_{k}$, until there are no electrons left. In particular, for $N=Z$, the infimum (3.15) is zero and the only minimizer (up to a trivial renumbering) is for $\rho=\bar{\rho}$. On the other hand, when $N>Z$, the infimum (3.15) is still zero, but is not attained: $N-Z$ excess electrons are pushed to infinity, so that $\rho=\bar{\rho}$ again. The necessary and sufficient condition for a minimizer to exist is therefore again (3.11).

In the same fashion, notice that the same condition as (3.11) exists for the $N$-body problem (2.8) in order to ensure the existence of an infinity of discrete eigenvalues below the essential spectrum; see [207]. 
Coming back to the HF model, let us also point out that condition (3.11) is only a sufficient condition. No necessary condition is known for the existence. The only result that is known in this direction is a result by Solovej in [191], improving a previous result by Lieb in [161], and stating the existence of some $m$ such that for $N \geq Z+m$ there never exists a minimizer.

Any more precise result even for a particular given pair $(N, Z)$ (say for $N$ small and $Z=1$, or for any arbitrary $N$ and $Z=N-1$ ) would be particularly useful and enlightening, but to the best of our knowledge no such result has been established.

In the same vein (and contrary to what is sometimes erroneously stated in the chemistry literature), nothing is known on any kind of uniqueness that may hold (up to the trivial invariance properties of the Hartree-Fock energy functional). For instance, a natural question is the uniqueness of the minimizing electronic density $\rho$. Any insight on such issues would have tremendous consequences, in particular on the numerics.

Finally, let us mention that the compactness of all minimizing sequences is a result slightly stronger than the existence of a minimizer, that has in particular an impact on the behaviour of the numerical approximations of the problem.

Let us now outline the proof of the above theorem.

It is simple to see that a minimizing sequence $\left(\phi_{1}^{n}, \ldots, \phi_{N}^{n}\right)$ is necessarily bounded in $H^{1}\left(\mathbb{R}^{3}\right)^{N}$, and that it therefore converges up to an extraction to some $N$-tuple $\left(\phi_{1}, \ldots, \phi_{N}\right)$. Studying each of its terms, one sees that the energy functional $E^{H F}$ is lower semi-continuous for the $H^{1}$ weak topology. On the other hand, the orthonormality constraint is a priori not preserved in the limit. It can therefore be claimed that

$$
E^{H F}\left(\phi_{1}, \ldots, \phi_{N}\right) \leq I_{N}^{H F} \quad \text { and } \quad 0 \leq\left[\int_{\mathbb{R}^{3}} \phi_{i} \phi_{j}\right] \leq\left[\delta_{i j}\right],
$$

in the sense of symmetric matrices, and no more than that a priori. In order to overcome the difficulty and see when $\left[\int_{\mathbb{R}^{3}} \phi_{i} \phi_{j}\right]=$ Id holds, we introduce the following family of minimization problems:

$$
I_{N}(A)=\inf \left\{E^{H F}\left(\phi_{1}, \ldots, \phi_{N}\right), \quad \phi_{i} \in H^{1}\left(\mathbb{R}^{3}\right), \quad\left[\int_{\mathbb{R}^{3}} \phi_{i} \phi_{j}\right]=A\right\},
$$

for any nonnegative symmetric matrix $A$. Of course, (3.7) corresponds to the case when $A$ is the identity matrix.

Then, the proof of Theorem 3.2 relies on two properties of monotonicity and concavity of the infimum $I_{N}(A)$ defined by (3.17) with respect to the constraint defined by $A$.

The property of monotonicity originates from physics. If some of the electrons are sent to infinity, the energy can only increase. This is easily seen by the following mathematical argument. Fix $\varepsilon>0$, fix two symmetric matrices $A_{1} \geq A_{2}$, and choose some $\left(\psi_{1}, \ldots, \psi_{N}\right)$, all compactly supported, such that $\left[\int_{\mathbb{R}^{3}} \psi_{i} \psi_{j}\right]=A_{2}$ and

$$
I_{N}\left(A_{2}\right) \leq E^{H F}\left(\psi_{1}, \ldots, \psi_{N}\right) \leq I_{N}\left(A_{2}\right)+\varepsilon .
$$

Now fix $\left(\chi_{1}, \ldots, \chi_{N}\right)$, again all compactly supported, such that $\left[\int_{\mathbb{R}^{3}} \chi_{i} \chi_{j}\right]=A_{1}-A_{2}$. Consider $\psi_{i}^{n}=\psi_{i}+n^{-3 / 2} \chi_{i}(\dot{\bar{n}})$ (this is a way to eliminate excess electrons). By 
definition of the infimum,

$$
I_{N}\left(\left[\int_{\mathbf{R}^{3}} \psi_{i}^{n} \psi_{j}^{n}\right]\right) \leq E^{H F}\left(\psi_{1}^{n}, \ldots, \psi_{N}^{n}\right),
$$

and it is simple to see that, as $n$ goes to infinity, the left-hand side converges to $I_{N}\left(A_{1}\right)$, while the right-hand side converges to $E^{H F}\left(\psi_{1}, \ldots, \psi_{N}\right)$. This shows that

$$
I_{N}\left(A_{1}\right) \leq E^{H F}\left(\psi_{1}, \ldots, \psi_{N}\right) \leq I_{N}\left(A_{2}\right)+\varepsilon,
$$

for all $\varepsilon>0$, and proves that $I_{N}(A)$ is nonincreasing with respect to $A$. In particular, we can now deduce from (3.16) that $E^{H F}\left(\phi_{1}, \ldots, \phi_{N}\right)=I_{N}^{H F}$.

Unlike the monotonicity property that stems from physics, the concavity property originates from the specific model at hand. We begin by noticing that we can always diagonalize the matrix $\left[\int_{\mathbf{R}^{3}} \psi_{i} \psi_{j}\right]$ without changing the energy $E^{H F}\left(\psi_{1}, \ldots\right.$, $\left.\psi_{N}\right)$. This is clear on the original expression of the Hartree-Fock energy: it is a function of the determinant of $\left(\psi_{1}, \ldots, \psi_{N}\right)$, and a determinant is invariant under orthogonal transformations. Therefore, when the $\lambda_{i}$ are the eigenvalues of the symmetric matrix $A$, we have

$$
\begin{aligned}
& I_{N}(A)= I_{N}\left(\lambda_{1}, \ldots, \lambda_{N}\right) \\
&= \inf \left\{E^{H F}\left(\psi_{1}, \ldots, \psi_{N}\right), \psi_{i} \in H^{1}\left(\mathbb{R}^{3}\right),\left[\int_{\mathbb{R}^{3}} \psi_{i} \psi_{j}\right]=\operatorname{diag}\left(\lambda_{1}, \ldots, \lambda_{N}\right)\right\} \\
&= \inf \left\{\sum_{i=1}^{N} \lambda_{i}\left(\frac{1}{2} \int_{\mathbb{R}^{3}}\left|\nabla \psi_{i}\right|^{2}+\int_{\mathbb{R}^{3}}\left|\psi_{i}\right|^{2} V\right)\right. \\
&+\frac{1}{2} \sum_{i \neq j} \lambda_{i} \lambda_{j} \int_{\mathbf{R}^{3}} \int_{\mathbb{R}^{3}} \frac{\left|\psi_{i}(x)\right|^{2}\left|\psi_{j}\left(x^{\prime}\right)\right|^{2}-\psi_{i}(x) \psi_{j}(x) \psi_{i}\left(x^{\prime}\right) \psi_{j}\left(x^{\prime}\right)}{\left|x-x^{\prime}\right|} d x d x^{\prime}, \\
&\left.\psi_{i} \in H^{1}\left(\mathbb{R}^{3}\right), \quad\left[\int_{\mathbf{R}^{3}} \psi_{i} \psi_{j}\right]=\delta_{i j}\right\} .
\end{aligned}
$$

When all the $\lambda_{i}$ are fixed except one, say $\lambda_{N}$, the energy one minimizes is linear with respect to $\lambda_{N}$. As an infimum of a linear function is concave, this shows the concavity of $I_{N}\left(\lambda_{1}, \ldots, \lambda_{N}\right)$ with respect to any of the $\lambda_{i}$.

On the basis of the above two properties, we now conclude the proof of Theorem 3.2. Let us denote by $1 \geq \lambda_{1} \geq \ldots \geq \lambda_{N} \geq 0$ the eigenvalues of the ma$\operatorname{trix}\left[\int_{\mathbf{R}^{3}} \phi_{i} \phi_{j}\right]$. Let us suppose that all $\lambda_{i}$ are not equal to 1 and denote by $i$ the smallest index in $\{1, \ldots, N\}$ such that $\lambda_{i}<1$. We thus have $I_{N}(1,1, \ldots, 1)=$ $I_{N}\left(1, \ldots, 1, \lambda_{i}, \ldots, \lambda_{N}\right)$. Because $I_{N}$ is both concave and nonincreasing, this shows that

$$
I_{N}\left(1, \ldots, 1, \lambda, \lambda_{i+1}, \ldots, \lambda_{N}\right)
$$

is a constant function of $\lambda \in[0,1]$. Therefore, repeating the argument for all $j$ from $i+1$ to $N$,

$$
I_{N}^{H F}=I_{N}(1,1, \ldots, 1)=I_{N}(1, \ldots, 1,0, \ldots, 0)=I_{N-i}^{H F} .
$$

This implies $I_{N}^{H F}=I_{N-1}^{H F}=I_{N-i}^{H F}$ and contradicts (3.10), thereby showing assertion (i) of Theorem 3.2

For assertion (ii) we proceed by induction on $N$. The case $N=1$ reduces to $I_{1}<0$, which is easily seen. We next fix $N$, assume $Z>N-1$ and $I_{N-1}<$ $I_{N-2}<\ldots<I_{1}<0$. With the same notation as above, we then claim that 
$I_{N} \leq I_{N}(1,1, \ldots, 1,1)<I_{N-1}(1,1, \ldots, 1)=I_{N-1}$, which shows assertion (ii) at rank $N$. For this purpose, we write

$$
\begin{aligned}
E^{H F}\left(\phi_{1}, \ldots, \phi_{N-1}, \varphi\right) & -I_{N-1}=\int|\nabla \varphi|^{2}+\int|\varphi|^{2} V \\
& +\iint \frac{\rho_{N-1}(x) \varphi^{2}\left(x^{\prime}\right)}{\left|x-x^{\prime}\right|}-\iint \frac{\rho_{N-1}\left(x, x^{\prime}\right) \varphi(x) \varphi\left(x^{\prime}\right)}{\left|x-x^{\prime}\right|},
\end{aligned}
$$

where $\left(\phi_{1}, \ldots, \phi_{N-1}\right)$ is a minimizer of $I_{N-1}$, and $\varphi$ an arbitrary function to be fixed below. It remains now to show that, under the condition $Z>N-1$, we may find some $N$-dimensional vector space (in order to ensure orthogonality to the first $N-1 \phi_{i}$ ) of functions $\varphi$ such that (3.19) is a negative quantity. This is indeed proven by considering a space of radially symmetric functions $\varphi$ that are adequately rescaled. The bottom line of the argument is, as announced above, some balance of electrostatic interaction: (forgetting the last term for clarity) the right-hand side of (3.19) involves the Schrödinger operator

$$
-\frac{1}{2} \Delta+V+\rho_{N-1} \star \frac{1}{|x|}
$$

whose potential $V+\rho_{N-1} \star \frac{1}{|x|}$ roughly behaves as $\frac{-Z+(N-1)}{|x|}$ at infinity. For $Z>N-1$, this potential is sufficiently negative at infinity so that negative eigenstates (and in fact infinitely many) exist. This concludes the proof of the theorem.

It is worth mentioning at this stage that the same proof, even slightly simpler and up to minor modifications, shows the following existence theorem on the Hartree model (abbreviated by $\mathrm{H}$ ). The Hartree model is a predecessor of the Hartree-Fock model when the antisymmetry requirement is ignored, or in other words the test functions are restricted to be of the form

$$
\psi_{e}\left(x_{1}, \ldots, x_{N}\right)=\prod_{i=1}^{N} \phi_{i}\left(x_{i}\right),
$$

for normalized $\phi_{i}$, in the minimization problem (2.7).

\section{Theorem 3.3. Existence of a minimizer for the $\mathbf{H}$ model}

Consider the Hartree model

$$
\begin{aligned}
& I_{N}^{H}= \inf \left\{E^{H}\left(\phi_{1}, \ldots, \phi_{N}\right)=\sum_{i=1}^{N} \frac{1}{2} \int_{\mathbb{R}^{3}}\left|\nabla \phi_{i}\right|^{2}+\int_{\mathbf{R}^{3}} \rho V\right. \\
&+ \frac{1}{2} \sum_{i \neq j} \int_{\mathbb{R}^{3}} \int_{\mathbb{R}^{3}} \frac{\left|\phi_{i}(x)\right|^{2}\left|\phi_{j}\left(x^{\prime}\right)\right|^{2}}{\left|x-x^{\prime}\right|} d x d x^{\prime}, \\
&\left.\phi_{i} \in H^{1}\left(\mathbb{R}^{3}\right), \quad \int_{\mathbb{R}^{3}}\left|\phi_{i}\right|^{2}=1, \quad 1 \leq i \leq N\right\} .
\end{aligned}
$$

- (i) Any minimizing sequence of (3.20) is relatively compact in $\left(H^{1}\left(\mathbb{R}^{3}\right)\right)^{N}$ if and only if the following condition holds:

$$
I_{N}^{H}<I_{N-1}^{H} .
$$


- (ii) Condition (3.21) holds as soon as the total nuclear charge satisfies

$$
Z=\sum_{k=1}^{M} z_{k}>N-1
$$

All of the comments we made after the statement of Theorem 3.2 on the HartreeFock model hold for the Hartree model.

3.1.4. The Euler-Lagrange equations. A minimizer of the Hartree-Fock problem satisfies the Euler-Lagrange equations associated to (3.7), namely the following system of $N$ coupled partial differential equations:

$$
\left\{\begin{array}{l}
-\frac{1}{2} \Delta \phi_{i}+V \phi_{i}+\left(\sum_{j=1}^{N}\left|\phi_{j}\right|^{2} \star \frac{1}{|x|}\right) \phi_{i}-\sum_{j=1}^{N}\left(\phi_{i} \phi_{j} \star \frac{1}{|x|}\right) \phi_{j}=\sum_{j=1}^{N} \lambda_{i j} \phi_{j}, \\
\int_{\mathbf{R}^{3}} \phi_{i} \phi_{j}=\delta_{i j}
\end{array}\right.
$$

where the matrix $\lambda_{i j}$ is the self-adjoint matrix of Lagrange multipliers associated to the orthonormality constraints.

Next, because of the orthogonal invariance of the Hartree-Fock energy functional mentioned above, we may diagonalize the matrix $\lambda_{i j}$ of Lagrange multipliers and obtain (with a slight abuse of notation) the Hartree-Fock equations:

$$
\left\{\begin{array}{l}
-\frac{1}{2} \Delta \phi_{i}+V \phi_{i}+\left(\sum_{j=1}^{N}\left|\phi_{j}\right|^{2} \star \frac{1}{|x|}\right) \phi_{i}-\sum_{j=1}^{N}\left(\phi_{i} \phi_{j} \star \frac{1}{|x|}\right) \phi_{j}=\lambda_{i} \phi_{i}, \\
\int_{\mathbf{R}^{3}} \phi_{i} \phi_{j}=\delta_{i j} .
\end{array}\right.
$$

The equations (3.24) can be written in the more compact form

$$
\left\{\begin{array}{l}
F_{\Phi} \phi_{i}=\lambda_{i} \phi_{i} \\
\int_{\mathbb{R}^{3}} \phi_{i} \phi_{j}=\delta_{i j}
\end{array}\right.
$$

with

$$
F_{\Phi}=-\frac{1}{2} \Delta+V+\left(\sum_{j=1}^{N}\left|\phi_{j}\right|^{2} \star \frac{1}{|x|}\right)-\sum_{j=1}^{N}\left(\cdot \phi_{j} \star \frac{1}{|x|}\right) \phi_{j} .
$$

They then appear as a nonlinear eigenvalue problem, known in quantum chemistry as a self-consistent field problem, abbreviated as SCF, in order to emphasize that the electrostatic field the electrons are experiencing indeed depends on the $\phi_{i} \mathrm{~s}$. Any minimizer to (3.7) is, up to an orthogonal transform, a solution to (3.24). Moreover, it has been proven in [166] that if $\Phi$ is a Hartree-Fock ground state, then the $\lambda_{i}$ necessarily are negative and are the lowest $N$ eigenvalues of the Fock operator $F_{\Phi}$. Another interesting property, established in [4], is that, for any minimizer, the $N$-th energy level $\lambda_{N}$ is not degenerate.

Because we do not dispose of any convexity property, it is not known whether any solution to (3.24) conversely is a minimizer to (3.7), which is a major difficulty for the numerical approximation of the problem. 
Due to standard elliptic theory results, the $\phi_{i}$ solutions to (3.24) are necessarily smooth functions (except at the point nuclei), that in addition decrease exponentially fast at infinity. This latter property agrees with the properties of the exact wavefunction recalled in Remark 2.2.

Let us also mention that, again, we may apply to the Hartree model the techniques of the Hartree-Fock model, in a more simple way though. Then the EulerLagrange equations read

$$
\left\{\begin{array}{l}
-\frac{1}{2} \Delta \phi_{i}+V \phi_{i}+\left(\sum_{j \neq i}\left|\phi_{j}\right|^{2} \star \frac{1}{|x|}\right) \phi_{i}=\lambda_{i} \phi_{i}, \\
\int_{\mathbf{R}^{3}}\left|\phi_{i}\right|^{2}=1,
\end{array}\right.
$$

where it can be shown that $\lambda_{i} \leq 0$, and, for $Z>N-1$, that $\lambda_{i}<0$. All remarks on the equivalence with the minimization viewpoint, the regularity and the exponential decay of the $\phi_{i}$ hold again.

3.1.5. Beyond Hartree-Fock. Many post Hartree-Fock methods exist in the chemical literature. As the Hartree-Fock approximation is a variational approximation of (2.7), i.e. an approximation constructed by restricting the variational space $\mathcal{H}_{e}$ to a smaller one, most of its improvements consist in enlarging the variational space.

An illustrative example is provided by the multiconfiguration self-consistent field method (abbreviated as MCSCF) that aims at recovering more generality on the wavefunction $\psi_{e}$ by minimizing on sums of determinants. The MCSCF problem can be stated as follows:

$$
\begin{gathered}
E_{N}^{K}=\inf \left\{\left\langle\psi_{e}, H_{e} \psi_{e}\right\rangle, \quad \psi_{e}=\sum_{I=\left\{i_{1}, \ldots, i_{N}\right\} \subset\{1, \ldots, K\}} c_{I} \frac{1}{\sqrt{N !}} \operatorname{det}\left(\phi_{i_{1}}, \ldots, \phi_{i_{N}}\right),\right. \\
\left.\phi_{i} \in H^{1}\left(\mathbb{R}^{3}\right), \quad \int_{\mathbb{R}^{3}} \phi_{i} \phi_{j}=\delta_{i j}, \quad \sum_{I} c_{I}^{2}=1\right\},
\end{gathered}
$$

where $K \geq N$ is some given fixed integer. The constraints on the $\phi_{i}$ and the $c_{I}$ amount to enforcing $\left\|\psi_{e}\right\|_{L^{2}}=1$ and $\operatorname{Rank}\left(\mathcal{D}_{\psi_{e}}\right) \leq K$, where $\mathcal{D}_{\psi_{e}}$ is the density operator built from $\psi_{e}$.

The Euler-Lagrange equations of this minimization problem can be derived in the same fashion as they are in the Hartree-Fock setting, and one then obtains the MCSCF equations, which basically are of the same form as the Hartree-Fock equations (3.24), apart from tedious technical details.

The MCSCF model (3.28) gives rise to the same theoretical interesting questions as the HF model does. The first mathematical results on the MCSCF model are due to one of us in [143], in the case of two determinants. This preliminary result has been considerably complemented in [114] to deal with the general case of many determinants. This general case is the full MCSCF model, stated exactly in (3.28). Alternatively, the discrete summation in (3.28) can possibly be restricted to some convenient subsets of $\{1, \ldots, K\}$. This gives rise to particular variants of the MCSCF model, that are indeed treated in practice, (3.28) being untractable for most systems of physical interest. The proofs of [114] have recently been simplified and the results further complemented and improved in [150], in order notably to apply to those practical cases. This latter work puts the mathematical knowledge on the MCSCF model on the same level as that on the HF model, which can be heuristically 
understood as a manifestation of the fact that both models come from successive degrees of variational approximation of the sole problem (2.7).

3.1.6. A parenthesis on relativistic models. In the case when the molecular system under study involves one or many heavy atoms, the relativistic effects are of major importance. Such a situation requires specific models, issued from relativistic quantum chemistry. Let us only mention here that, neglecting the relativistic effects for heavy atoms and modelling them in the nonrelativistic framework we have dealt with so far, may lead to incorrect physical conclusions. For instance, modelling a heavy atom in a nonrelativistic setting leads to the conclusion that gold is not yellow or that mercury is not a liquid metal.

We will briefly review in this section some elements of modelling for the relativistic situation, the mathematical side of which has been recently investigated by a series of work by Esteban, Séré and collaborators (see [98] for a review by these authors).

What plays the role of the Schrödinger Hamiltonian for a relativistic electron of coordinates $\left(x_{1}, x_{2}, x_{3}\right) \in \mathbb{R}^{3}$ is the Dirac Hamiltonian:

$$
H_{c}=-i \alpha_{1} \frac{\partial}{\partial x_{1}}-i \alpha_{2} \frac{\partial}{\partial x_{2}}-i \alpha_{3} \frac{\partial}{\partial x_{3}}+c^{2} \beta,
$$

where $c$ is the speed of light ( $c=137$ in the atomic unit system we have adopted so far, and recall that the mass of the electron is $m=1$ in this system), $\alpha_{k}, k=1,2,3$, and $\beta$ are $4 \times 4$ matrices given by

$$
\alpha_{k}=\left(\begin{array}{cc}
0 & \sigma_{k} \\
\sigma_{k} & 0
\end{array}\right), \quad \beta=\left(\begin{array}{cc}
\mathrm{Id}_{2} & 0 \\
0 & -\mathrm{Id}_{2}
\end{array}\right),
$$

where $\operatorname{Id}_{2}$ is the $2 \times 2$ identity matrix, and the $\sigma_{k}$ are the Pauli matrices

$$
\sigma_{1}=\left(\begin{array}{cc}
0 & 1 \\
1 & 0
\end{array}\right), \quad \sigma_{2}=\left(\begin{array}{cc}
0 & -i \\
i & 0
\end{array}\right), \quad \sigma_{3}=\left(\begin{array}{cc}
1 & 0 \\
0 & -1
\end{array}\right)
$$

The introduction of this Hamiltonian, by Dirac, is motivated by the fact that $H_{c}^{2}$ needs to be equal to the operator $-c^{2} \Delta+c^{4}$, which is the quantum analogue of the Hamiltonian of classical relativity $p^{2} c^{2}+c^{4}$ (where $p$ is the momentum operator), exactly as the Schrödinger operator (2.3) is the analogous operator to (2.4). Dirac constructed $H_{c}$ as a simple differential operator (as implied by relativity theory) that has constant coefficients (in order to be invariant under the transformations of the Lorentz group) and gives the correct square $H_{c}^{2}$.

The Dirac Hamiltonian $H_{c}$ acts on 4 -spinors, i.e. wavefunctions valued in $\mathbb{C}^{4}$ (and not $\mathbb{C}$ or $\mathbb{R}$ as was the case in nonrelativistic quantum mechanics). Its domain is $H^{1}\left(\mathbb{R}^{3}, \mathbb{C}^{4}\right)$ and its (entirely continuous) spectrum is $\left.\left.\sigma\left(H_{c}\right)=\right]-\infty,-c^{2}\right] \cup\left[c^{2},+\infty[\right.$. The striking difference with the Laplacian operator, whose spectrum is $[0,+\infty[$, lies in the fact that the spectrum of the Dirac Hamiltonian is not bounded from below. This will have devastating consequences on the mathematical analysis of the minimization problems we shall deal with. They will therefore have a continuum of negative directions at a critical point. So minimization will have to be replaced by adequate saddle-point methods.

If we now place this electron in the field of a nucleus of charge $Z$ placed at the origin of $\mathbb{R}^{3}$, the Hamiltonian reads

$$
H_{Z, c}=H_{c}-\frac{Z}{|x|}
$$


(the case of a molecule can be dealt with, replacing the potential $-Z /|x|$ by $V$ defined by (2.5)). When $Z<c$, it can be shown that $H_{Z, c}$ has a unique self-adjoint extension, still denoted by $H_{Z, c}$. Its spectrum is

$$
\left.\left.\sigma\left(H_{Z, c}\right)=\right]-\infty,-c^{2}\right] \cup\left\{\lambda_{1}^{c}, \ldots, \lambda_{k}^{c}, \ldots\right\} \cup\left[c^{2},+\infty[\right.
$$

as the potential $-Z /|x|$ has indeed created discrete eigenvalues in the gap $]-c^{2}, c^{2}[$ of the spectrum of $H_{c}$. The discrete eigenvalues $\lambda_{k}^{c}$ form an increasing sequence that starts from the lowest eigenvalue $\lambda_{1}^{c}=\sqrt{c^{4}-c^{2} Z^{2}}$ and converges to $c^{2}$ as $k$ goes to infinity. To each of them corresponds an eigenstate $\psi_{k}^{c}=\left(\begin{array}{c}\varphi_{k}^{c} \\ \chi_{k}^{c}\end{array}\right)$ (with $\varphi_{k}^{c}$ and $\chi_{k}^{c}$ valued in $\mathbb{C}^{2}$ ). When $c$ goes to infinity, one has

$$
\lambda_{k}^{c}-c^{2} \longrightarrow \epsilon_{k}, \quad \varphi_{k}^{c} \longrightarrow \varphi_{k}, \quad \chi_{k}^{c} \longrightarrow 0,
$$

where $\left(\varphi_{k}, \epsilon_{k}\right)$ is the $k$-th (eigenfunction, eigenvalue) pair for the classical operator $-\frac{1}{2}-\Delta-Z /|x|$. This can be seen as a consistency result connecting the relativistic setting with the nonrelativistic one.

As announced above, the huge technical difficulties originating from the unboundedness from below of the Dirac Hamiltonian have long been an obstacle to a variational characterization of the eigenvalues of the operator $H_{Z, c}$ (and further to a rigorously founded numerical approach consisting of directly attacking the search for eigenvalues and eigenfunctions variationally).

The situation was only recently settled by the following.

Theorem 3.4. 85, 86 Minimax characterization of the eigenvalues of the atomic Dirac Hamiltonian

Assume $Z<c$. Then,

$$
\lambda_{k}^{c}=\inf _{\substack{V \subset \Lambda^{+} C_{0}^{\infty}\left(\mathbb{R}^{3}, \mathbb{C}^{4}\right) \\ \operatorname{dim} V=k}} \sup _{\substack{\psi \in V \oplus \Lambda^{-} C_{0}^{\infty}\left(\mathbf{R}^{3}, \mathbb{C}^{4}\right) \\ \int_{\mathbf{R}^{3}}|\psi|^{2}=1}}\left\langle\psi, H_{Z, c} \psi\right\rangle,
$$

where $\Lambda^{+}$and $\Lambda^{-}$respectively denote the positive and negative projectors of $H_{Z, c}$. by

$$
\begin{gathered}
\lambda_{k}^{c}=\inf _{\substack{W \subset C_{0}^{\infty}\left(\mathbb{R}^{3}, \mathbb{C}^{2}\right) \\
\operatorname{dim} W=k}} \sup _{\substack{\varphi \in W \\
\chi \in C_{0}^{\infty}\left(\mathbb{R}^{3}, \mathbb{C}^{2}\right)}}\left\langle\psi, H_{Z, c} \psi\right\rangle . \\
\psi=\left(\begin{array}{c}
\varphi \\
\chi
\end{array}\right)
\end{gathered}
$$

This characterization is again valid for $Z<c$ and is based upon previous formal ideas from [200] and 77].

These kinds of minimax characterizations have given rise to new algorithmic techniques to compute the eigenfunctions and eigenvalues of the Dirac operator in molecules [87, 84, 88, 83. But, as is the case in the nonrelativistic setting, it is only possible to attack a system of $N$ electrons modelled by the Dirac Hamiltonian when $N$ is dramatically small. Approximations have thus been developed, and one of the most commonly used is the Dirac-Fock approximation, which is the relativistic version, introduced in [197, 198], of the Hartree-Fock approximation.

There seems to be no rigorous physical ground known to date for this approximation that formally consists of building the energy functional $E^{D F}\left(\psi_{1}, \ldots, \psi_{N}\right)$ by 
simply replacing mutatis mutandis the kinetic energy of the HF energy, namely the first term of (3.6):

$$
\sum_{i=1}^{N} \frac{1}{2} \int_{\mathbb{R}^{3}}\left|\nabla \phi_{i}\right|^{2}=\sum_{i=1}^{N} \frac{1}{2} \int_{\mathbf{R}^{3}}-\Delta \phi_{i} \phi_{i}
$$

by that obtained with the Dirac Hamiltonian

$$
\sum_{k=1}^{N} \frac{1}{2} \int_{\mathbf{R}^{3}}\left\langle\psi_{k}, H_{c} \psi_{k}\right\rangle,
$$

again for an orthonormal family: $\int_{\mathbb{R}^{3}} \psi_{k} \psi_{l}=\delta_{k l}$.

The Euler-Lagrange equations of the Dirac-Fock problem are derived in the same fashion as the Hartree-Fock equations (3.6) and are called the Dirac-Fock equations.

Theorem 3.5. [97, 99, 179] Existence of solutions to the Dirac-Fock equations

Suppose $Z \leq N, Z<0.9 c$ and $N<0.9 c$. Then there exists a sequence of solutions $\left(\psi^{j}, j \geq 1\right)$ to the Dirac-Fock equations with $0<\lambda_{j}^{k}<c^{2}$ for all $1 \leq k \leq$ $N, 0<E^{D F}\left(\psi^{j}\right)<N c^{2}$ that satisfies

$$
E^{D F}\left(\psi^{j}\right) \stackrel{j \longrightarrow+\infty}{\longrightarrow} N c^{2} .
$$

Let us point out that the condition $N<0.9 c$ is a highly nontrivial extension by Paturel in [179] of a stronger condition assumed earlier by Esteban and Séré. As such, this condition ensures that the theorem holds for any $N<124$, which covers all existing atoms.

Remark 3.6. Notice that this condition is valid for molecules consisting of more than one atom, but then it does not cover all physically relevant cases. A mathematical argument, roughly speaking bounding the "number of electrons per atom" in the molecule, would suffice to settle the situation mathematically. But to date, such an argument is still missing.

The following connection between the DF model and the HF model can be established.

\section{Theorem 3.7. 100 Hartree-Fock obtained as a limit of Dirac-Fock}

Suppose $Z \geq N$ and $j$ is fixed. Then as $c$ goes to infinity, the solutions $\psi^{j}=$ $\left(\begin{array}{l}\varphi^{j} \\ \chi^{j}\end{array}\right)$ with eigenvalues $\left(\lambda_{1}^{j}, \ldots, \lambda_{N}^{j}\right)$ issued from Theorem 3.5 satisfy:

$$
\varphi_{k}^{j} \stackrel{c \longrightarrow+\infty}{\longrightarrow} \phi_{k}^{j}, \quad \chi_{k}^{j} \stackrel{c \longrightarrow+\infty}{\longrightarrow} 0, \quad \lambda_{k}^{j}-c^{2} \stackrel{c \longrightarrow+\infty}{\longrightarrow} \epsilon_{k}^{j}<0,
$$

where $\left(\phi_{1}^{j}, \ldots, \phi_{N}^{j}\right)$ is a solution to the Hartree-Fock equations associated to the eigenvalues $\left(\epsilon_{1}^{j}, \ldots, \epsilon_{N}^{j}\right)$. In addition, the case $j=1$ corresponds to a minimizer of the Hartree-Fock energy.

The consistency of the Dirac-Fock model with respect to the Hartree-Fock model stated in the above theorem (and extending to the nonlinear setting the convergences (3.34) ) can be seen as one of the rare fundamental justifications of the Dirac-Fock model. In addition, the last assertion of Theorem 3.7 suggests that $\psi^{1}$ is a good candidate to be the ground state of the Dirac-Fock model, a notion that so far was not clearly defined in the literature. In this direction, the minimax 
characterization obtained in the linear case in Theorem 3.4 was extended to the nonlinear setting, thereby allowing for a characterization of the ground state in the Dirac-Fock model.

Theorem 3.8. 102] Minimax characterization of the Dirac-Fock ground state

Suppose $Z \geq N$. Then, for c large enough, $\psi_{1}$ given by Theorem 3.5 is a DiracFock ground state in the following sense:

$$
E^{D F}\left(\psi_{1}\right)=\left\{\begin{array}{l}
\psi=\left(\psi_{1}, \ldots, \psi_{N}\right) \\
\psi_{k} \in H^{1 / 2}\left(\mathbb{R}^{3}, \mathbb{C}^{4}\right) \\
\int_{\mathbb{R}^{3}} \psi_{k} \psi_{l}=\delta_{k l} \\
\Lambda_{\psi}^{-} \psi_{k}=0, \quad \text { for all } 1 \leq k \leq N
\end{array} E^{D F}(\psi)\right.
$$

where $\Lambda_{\psi}^{-}$denotes the negative projector of the (self-consistent) Dirac operator $H_{\psi}^{c}$.

We point out the crucial role played by the condition $\Lambda_{\psi}^{-} \psi_{k}=0$, for all $1 \leq k \leq$ $N$ that prevents the minimization from collapsing to $-\infty$. Contrary to previous approaches of the chemistry literature, this is not imposed a priori with a fixed operator but in a self-consistent way, which clearly improves the mathematical and the physical contents of the definition.

This definition in turn allows for very promising numerical approaches for the calculations of Dirac-Fock states [88, 83.

Let us conclude this section by mentioning that, as in the Hartree-Fock case, the Dirac-Fock model may be improved by considering a sum of determinants, thereby giving birth to the multiconfiguration Dirac-Fock model.

\subsection{Thomas-Fermi-type models.}

3.2.1. Physical modelling. Let us go back to the original electronic minimization problem (2.3)-(2.7) that we reproduce here for convenience:

$$
\begin{gathered}
U\left(\bar{x}_{1}, \cdots, \bar{x}_{M}\right)=\inf \left\{\left\langle\psi_{e}, H_{e} \psi_{e}\right\rangle, \quad \psi_{e} \in \mathcal{H}_{e}, \quad\left\|\psi_{e}\right\|_{L^{2}}=1\right\}, \\
H_{e}=-\sum_{i=1}^{N} \frac{1}{2} \Delta_{x_{i}}-\sum_{i=1}^{N} \sum_{k=1}^{M} \frac{z_{k}}{\left|x_{i}-\bar{x}_{k}\right|}+\sum_{1 \leq i<j \leq N} \frac{1}{\left|x_{i}-x_{j}\right|} .
\end{gathered}
$$

The purpose of the density functional theory, abbreviated as DFT, is to replace the minimization problem (2.7) defined in terms of the unknown wavefunction $\psi_{e}$ by a minimization problem set on the unknown density $\rho$.

To fulfill this goal, it suffices to introduce the functional

$$
\begin{array}{r}
E(\rho)=\inf \left\{\left\langle\psi_{e},\left(-\sum_{i=1}^{N} \frac{1}{2} \Delta_{x_{i}}+\sum_{1 \leq i<j \leq N} \frac{1}{\left|x_{i}-x_{j}\right|}\right) \psi_{e}\right\rangle,\right. \\
\left.\psi_{e} \in \mathcal{H}_{e}, \quad\left\|\psi_{e}\right\|_{L^{2}}=1, \quad \psi_{e} \text { has density } \rho\right\},
\end{array}
$$

on

$$
\mathcal{I}_{N}=\left\{\rho \geq 0, \quad \sqrt{\rho} \in H^{1}\left(\mathbb{R}^{3}\right), \quad \int_{\mathbb{R}^{3}} \rho=N\right\}
$$


so that

$$
U\left(\bar{x}_{1}, \cdots, \bar{x}_{M}\right)=\inf \left\{E(\rho)-\int\left(\sum_{k=1}^{M} \frac{z_{k}}{\left|\cdot-\bar{x}_{k}\right|}\right) \rho ; \quad \rho \in \mathcal{I}_{N}\right\} .
$$

The functional $E$ is the density functional. That given by (3.40) is called the LevyLieb density functional. Actually, other manipulations can be made on the energy (3.38) so that other problems of the type (3.42) can be defined, for functionals $E$ different from (3.40) and variational spaces different from $\mathcal{I}_{N}$. All this is related to the so-called Hohenberg and Kohn Theorem in quantum chemistry. For more details on the theory seen from the chemistry viewpoint, we refer the reader to the treatises [91, 178, and [173, and the review articles [160, 131, 136].

It is to be mentioned at this point that finding an explicit expression of a functional of type $E$, such as for instance that given by (3.40), for a given $\rho$ is of course an open problem. In some cases, the definition of the variational space is also an open issue.

Remark 3.9. We would like to mention an interesting mathematical approach to the above problem. It is studied in 33 34, 35] and other works by the same authors and is based upon a convenient description of the set of $\psi_{e}$ which have density $\rho$. It consists of decomposing the information contained in a state $\psi_{e}$ into one piece of information contained in the density $\rho$ itself and another contained in a state with constant density. The density being fixed, one therefore optimizes only upon the latter to evaluate $E(\rho)$. It seems however to be unclear how to efficiently implement the approach.

In practice, approximations of the density functional $E$ have been developed. These approximations rely on exact or very accurate evaluations of the density functional for reference systems "close" to the real system under study. One of these approximations (see Remark 3.10 for another one) consists of using as a reference system the uniform noninteracting electron gas. This is the Thomas-Fermi theory, which can indeed be seen as an ancestor, dating back to the 1920s, of the DFT.

For more details on this theory, we refer to the review article [159], which collects the theoretical results known in the early 1980s. Other interesting review articles, with more emphasis on the chemical issues are 192, 131]. In addition, we refer to [201, volume 4].

For a uniform noninteracting electron gas, the kinetic energy may be computed analytically:

$$
T_{T F}(\rho)=C_{T F} \int_{\mathbf{R}^{3}} \rho(x)^{5 / 3} d x,
$$

where $C_{T F}=\frac{10}{3}\left(3 \pi^{2}\right)^{2 / 3}$ denotes the Thomas-Fermi constant. It is to be noted that the exponent $\frac{5}{3}$, standard in statistical mechanics, can be understood mathematically as the only possible exponent $p$ when one wishes to consistently approximate the kinetic energy term $\int|\nabla \Phi|^{2}$ with $|\Phi|^{2}=\rho$ by a term of the form $\int \rho^{p}$ : simply change $\Phi$ into $\Phi_{\lambda}(\cdot)=\lambda^{3 / 2} \Phi(\lambda \cdot)$, leaving invariant the normalization constraint $\int\left|\Phi_{\lambda}(\cdot)\right|^{2}=1$ for all $\lambda$, and compare $\int\left|\nabla \Phi_{\lambda}\right|^{2}=\lambda^{2} \int|\nabla \Phi|^{2}$ with 
$\int\left|\Phi_{\lambda}\right|^{2 p}=\lambda^{3 p-3} \int|\Phi|^{2 p}$. It has also been established that a term of the form (3.43) bounds from below the kinetic energy of a system of fermions. In this respect, the best possible inequality known to date is the Lieb-Thirring inequality (see e.g. 162,152 ).

On the other hand, the interelectronic repulsion energy of such a gas may be approximated in a reasonable way by the electrostatic self-interaction energy of a classical charge distribution of density $\rho$ :

$$
J(\rho)=\frac{1}{2} \int_{\mathbf{R}^{3}} \int_{\mathbf{R}^{3}} \frac{\rho(x) \rho(y)}{|x-y|} d x d y .
$$

The Thomas-Fermi model therefore consists of adopting the two formulas (3.43)(3.44) as approximations for the system under study and thus in considering the minimization of the functional

$$
E_{T F}(\rho)=C_{T F} \int_{\mathbb{R}^{3}} \rho^{5 / 3}+\int_{\mathbf{R}^{3}} \rho V+\frac{1}{2} \int_{\mathbb{R}^{3}} \int_{\mathbf{R}^{3}} \frac{\rho(x) \rho(y)}{|x-y|} d x d y
$$

over all admissible densities $\rho$, which here describe the set

$$
\left\{\rho \geq 0, \quad \rho \in L^{1} \cap L^{5 / 3}\left(\mathbb{R}^{3}\right), \quad \int_{\mathbb{R}^{3}} \rho=N\right\} .
$$

Pursuing the idea of taking as a reference system the uniform noninteracting electron gas, Dirac computed the so-called exchange energy term, that is, a correction to the excess evaluation (3.44) of the electronic repulsion

$$
E_{x c}(\rho)=-C_{D} \rho^{4 / 3}
$$

where $C_{D}=\frac{3}{4}\left(\frac{3}{\pi}\right)^{1 / 3}$ denotes the Dirac constant. Again, the exponent $\frac{4}{3}$ is the only possible one when approximating an interaction term of the type (3.44) by a term $\int \rho^{p}$, and there is a proof that a term of this type bounds from below the exchange energy; see [157 158]. This gives the Thomas-Fermi-Dirac functional

$$
E_{T F D}(\rho)=C_{T F} \int_{\mathbb{R}^{3}} \rho^{5 / 3}+\int_{\mathbf{R}^{3}} \rho V+\frac{1}{2} \int_{\mathbb{R}^{3}} \int_{\mathbb{R}^{3}} \frac{\rho(x) \rho(y)}{|x-y|} d x d y-C_{D} \int_{\mathbf{R}^{3}} \rho^{4 / 3} .
$$

In addition, a correction term, due to von Weizsäcker and expressed in terms of $\int_{\mathbf{R}^{3}}|\nabla \sqrt{\rho}|^{2}$, can be added to the kinetic energy. This term is obtained by studying perturbations generated by small heterogeneities of the density, and respectively leads to the Thomas-Fermi-von Weizsäcker model

$$
\begin{aligned}
E_{T F W}(\rho)= & C_{W} \int_{\mathbb{R}^{3}}|\nabla \sqrt{\rho}|^{2}+C_{T F} \int_{\mathbb{R}^{3}} \rho^{5 / 3}+\int_{\mathbb{R}^{3}} \rho V \\
& +\frac{1}{2} \int_{\mathbb{R}^{3}} \int_{\mathbb{R}^{3}} \frac{\rho(x) \rho(y)}{|x-y|} d x d y,
\end{aligned}
$$

where $C_{W}$ is a universal contant, and the Thomas-Fermi-Dirac-von Weizsäcker model

$$
\begin{aligned}
E_{T F D W}(\rho)= & C_{W} \int_{\mathbb{R}^{3}}|\nabla \sqrt{\rho}|^{2}+C_{T F} \int_{\mathbb{R}^{3}} \rho^{5 / 3}+\int_{\mathbf{R}^{3}} \rho V \\
& +\frac{1}{2} \int_{\mathbb{R}^{3}} \int_{\mathbb{R}^{3}} \frac{\rho(x) \rho(y)}{|x-y|} d x d y-C_{D} \int_{\mathbb{R}^{3}} \rho^{4 / 3},
\end{aligned}
$$


both models requiring the $H^{1}$ regularity of $\sqrt{\rho}$. Each of the energy functionals $E_{T F}, E_{T F D}, E_{T F W}, E_{T F D W}$ gives birth to a minimization problem

$$
I_{N}^{T F, T F D}=\inf \left\{E_{T F, T F D}(\rho), \quad \rho \geq 0, \quad \rho \in L^{1} \cap L^{5 / 3}\left(\mathbb{R}^{3}\right), \quad \int_{\mathbb{R}^{3}} \rho=N\right\}
$$

or

$$
I_{N}^{T F W, T F D W}=\inf \left\{E_{T F W, T F D W}(\rho), \quad \rho \geq 0, \quad \sqrt{\rho} \in H^{1}\left(\mathbb{R}^{3}\right), \quad \int_{\mathbb{R}^{3}} \rho=N\right\} .
$$

From the standpoint of physics, Thomas-Fermi-like models allow one to recover qualitatively many fundamental physical properties. This has justified a constant interest. We send the reader to the bibliography quoted above for more information.

From the standpoint of mathematics, the Thomas-Fermi-type models, and especially those which contain the von Weizsäcker term, constitute an excellent toy model to elaborate mathematical arguments in a simple setting. These arguments can then be extended, in a second stage, to more commonly used models such as the Hartree-Fock-type models.

In the numerical practice, however, the Thomas-Fermi-type models are not used that much, for they are most often considered out-of-date. The commonly accepted statement is that such models give a correct, however approximate, description. It is often not sufficient for the accuracy needed in the applications of chemistry: see e.g. [71 for a discussion, and Figure 1 1 for some representative results. Nevertheless, some groups are now developing the orbital free models (see e.g. [59]), which are updated versions of, and refinements of, Thomas-Fermi-like models: with a view to reducing the computational cost, the electronic state is represented by one scalar field on $\mathbb{R}^{3}$ (the density, or often in practice its square root), as in TF-type models. Such methods seem very promising for the computations of large scale systems, at least when accuracy is not the primary concern, only a rough approximation of the electronic structure being necessary.

\begin{tabular}{|l||l|l|l|l|l|l|}
\hline & TF & TFD & TFD $\lambda$ W & KS-LDA & HF & Experiment \\
\hline $\mathrm{Ne}$ & -165.61 & -176.3 & -128.83 & -128.12 & -128.55 & -128.94 \\
\hline $\mathrm{Ar}$ & -652.72 & -680.7 & -529.94 & -525.85 & -526.82 & -527.60 \\
\hline
\end{tabular}

Figure 1. Numerical results reported in the literature for the total energy (atomic units) of two atomic systems, depending on the models used. The TFD $\lambda \mathrm{W}$ result refers to a TFDW-type model where the coefficient $C_{W}$ of the definition of $E_{T F D W}(\rho)$, commonly denoted by $\frac{1}{8} \lambda$, is adjusted (the result shown here is the best result that is obtained for two different values of $\lambda$ in the two systems mentioned here). The KS-LDA result is obtained with the KohnSham model with a Local Density Approximation for the correlation term (see Remark 3.10). The HF result is obtained in a very large basis set. It is to be noted that post-Hartree-Fock methods such as those described in Section 3.1.5 significantly improve the HF results and almost perfectly fit to the experimental data. All values are extracted from [178. 


\section{Remark 3.10. Kohn-Sham models}

Another option for the approximation of the density functional, and more specifically that of its kinetic energy term, is the model introduced by Kohn and Sham. Their idea to take as the reference system a system of $N$ noninteracting electrons made tractable the DFT approach. Under convenient assumptions, the kinetic energy of such a system reads

$$
T_{K S}(\rho)=\inf \left\{\frac{1}{2} \sum_{i=1}^{N} \int_{\mathbb{R}^{3}}\left|\nabla \phi_{i}\right|^{2}, \phi_{i} \in H^{1}\left(\mathbb{R}^{3}\right), \int_{\mathbf{R}^{3}} \phi_{i} \phi_{j}=\delta_{i j}, \sum_{i=1}^{N}\left|\phi_{i}\right|^{2}=\rho\right\} .
$$

This expression is then chosen as an approximation of the kinetic energy term for the system of interacting electrons under study, and one considers the now famous Kohn-Sham model

$$
\begin{gathered}
I_{N}^{K S}=\inf \left\{\frac{1}{2} \sum_{i=1}^{N} \int_{\mathbb{R}^{3}}\left|\nabla \phi_{i}\right|^{2}+\int \rho V+\frac{1}{2} \int_{\mathbb{R}^{3}} \int_{\mathbb{R}^{3}} \frac{\rho(x) \rho(y)}{|x-y|} d x d y+E_{x c}(\rho),\right. \\
\left.\phi_{i} \in H^{1}\left(\mathbb{R}^{3}\right), \quad \int_{\mathbb{R}^{3}} \phi_{i} \phi_{j}=\delta_{i j}\right\},
\end{gathered}
$$

where $\rho$ is a notation for $\sum_{i=1}^{N}\left|\phi_{i}\right|^{2}$. Approximations of the exchange term $E_{x c}(\rho)$ are then developed for different situations. A standard approximation is the local density approximation (LDA) for which $E_{x c}(\rho)=\int_{\mathbf{R}^{3}} F(\rho)$ for some $F(\rho)$ (for instance $F(\rho)=-\rho^{4 / 3}$ as in the Dirac term), but other more precise expressions have been developed. As in the Thomas-Fermi model, Kohn-Sham LDA models use the uniform electron gas as a reference system, but then only for a small part of the energy, so that the results are far better and the model is of much greater practical relevance. It seems fair to say that $50 \%$ of the ab initio calculations of molecular systems, and $90 \%$ of those of large systems, are today performed with Kohn-Shamtype models. However, we will not devote more time on it mathematically. The reason for this is that this approximation leads to variational problems whose mathematical nature is in some vague sense a mixing of that of Hartree-Fock-type models (because of the form of the kinetic energy term) and that of Thomas-Fermi-type models (because of the form of $E_{x c}(\rho)$ ). The mathematical arguments needed to treat such a model are inspired by those for either model (see [140]).

3.2.2. Existence of the ground state for simple models. We will mainly consider here the TF and the TFW models. When accounting for the Dirac term, namely in the TFD and TFDW model, the nature of the mathematical analysis radically changes, because the Dirac term is a concave term that breaks the convexity of the TF and TFW model respectively. Regarding the TFD model, some trick can be used (see [156, 159]) so that the techniques for the TF case can be carried through to the TFD case. On the contrary, when coming to the TFDW model, new tools are necessary, and we will briefly come to these at the end of Section 4 .

For convenience, we now recall the TF and the TFW models. For reasons that will be clear below, we only modify the formulae (3.46)-(3.47) given in the previous 
section by considering a constraint $\int_{\mathbb{R}^{3}} \rho$ that can take any real value $\lambda$ and not only integer values $N$. Therefore

$$
I_{\lambda}^{T F}=\inf \left\{E_{T F}(\rho), \quad \rho \geq 0, \quad \rho \in L^{1} \cap L^{5 / 3}\left(\mathbb{R}^{3}\right), \quad \int_{\mathbb{R}^{3}} \rho=\lambda\right\},
$$

where

$$
E_{T F}(\rho)=C_{T F} \int_{\mathbf{R}^{3}} \rho^{5 / 3}+\int_{\mathbf{R}^{3}} \rho V+\frac{1}{2} \int_{\mathbf{R}^{3}} \int_{\mathbf{R}^{3}} \frac{\rho(x) \rho(y)}{|x-y|} d x d y
$$

and

$$
I_{\lambda}^{T F W}=\inf \left\{E_{T F W}(\rho), \quad \rho \geq 0, \quad \sqrt{\rho} \in H^{1}\left(\mathbb{R}^{3}\right), \quad \int_{\mathbf{R}^{3}} \rho=\lambda\right\}
$$

where

$$
E_{T F W}(\rho)=C_{W} \int_{\mathbb{R}^{3}}|\nabla \sqrt{\rho}|^{2}+E_{T F}(\rho) .
$$

Precisely because of the convexity (and in fact the strict convexity) of the energy functionals with respect to $\sqrt{\rho}$ (together with the linearity of the constraint $\int_{\mathbf{R}^{3}} \rho=\lambda$ ), the mathematical analysis proceeds from (now) standard arguments and provides a complete understanding of the models. The main body of mathematical work on this theory is due to E. H. Lieb and collaborators in the 1970s-1980s.

The main result concerning the existence (and also nonexistence) of minimizers is contained in the following.

Theorem 3.11. [156] 14] Existence and nonexistence of minimizers for the TF and TFW models

For both TF and TFW, there exists some $0<\lambda_{c}<+\infty$ such that

- when $\lambda \leq \lambda_{c}$ there exists a unique minimizer,

- when $\lambda>\lambda_{c}$ no minimizer exists, and in fact the infimum (3.50) (respectively (3.52) ) is then uniquely attained at the minimizer $\rho_{\lambda_{c}}$ of $I_{\lambda_{c}}^{T F}$ (respectively $\left.I_{\lambda_{c}}^{T F W}\right)$. In addition

- for the TF model, $\lambda_{c}=Z$ (from [156]),

- for the TFW model, $\lambda_{c}>Z$ (from [14]).

The proof of the above theorem can be outlined as follows. First, the energy functional $E^{T F}$ and $E^{T F W}$ are strictly convex functions of $\rho$. The only nonstandard terms are respectively the interaction term

$$
D(\rho, \rho)=\int_{\mathbf{R}^{3}} \int_{\mathbb{R}^{3}} \frac{\rho(x) \rho(y)}{|x-y|}
$$

(note that we have already introduced this notation above in (3.12)), which can be rewritten as

$$
D(\rho, \rho)=\int_{\mathbb{R}^{3}}|\nabla \phi|^{2}, \text { with }-\Delta \phi=4 \pi \rho,
$$

thus its convexity, and the von Weizsäcker term $W(\rho)=\int_{\mathbb{R}^{3}}|\nabla \sqrt{\rho}|^{2}$, which can be shown to be strictly convex by various means. An elementary proof goes by noticing that

$$
\int_{\mathbf{R}^{3}}|\nabla \sqrt{\rho}|^{2}=\frac{1}{4} \int_{\mathbf{R}^{3}} \frac{|\nabla \rho|^{2}}{\rho}
$$


the latter being convex since $(a, b) \longrightarrow a^{2} / b$ is a convex function on $\mathbb{R} \times \mathbb{R}_{+}$. However, what may be the most illustrative argument is to note that $W(\rho)$ is a differentiable positively homogeneous function; thus the convexity condition $W(\rho) \geq$ $W(\tilde{\rho})+\left(W^{\prime}(\tilde{\rho}), \rho-\tilde{\rho}\right)$ for all $\rho$ and $\tilde{\rho}$ amounts to

$$
W(\rho) \geq\left(W^{\prime}(\rho), \tilde{\rho}\right), \quad \text { i.e. } \quad \int_{\mathbb{R}^{3}}|\nabla \sqrt{\rho}|^{2} \geq \int_{\mathbb{R}^{3}} \nabla \sqrt{\rho} \cdot \nabla\left(\frac{\tilde{\rho}}{\sqrt{\rho}}\right),
$$

which is simply saying that

$$
\int_{\mathbb{R}^{3}}\left|\nabla \log \left(\frac{\rho}{\tilde{\rho}}\right)\right|^{2} \rho \geq 0
$$

As suggested by this latter expression, the von Weizsäcker term is intimately related to Information Theory, since it is indeed connected to a relative entropy dissipation term.

Once this strict convexity of the energy functional is established, the uniqueness of a minimizer follows at once, and it remains to show its existence. For this purpose, it suffices to first remark that, sending some mass at infinity, the same argument as in the proof for the HF case shows that $I_{\lambda}^{T F}$ and $I_{\lambda}^{T F W}$ are nonincreasing with respect to $\lambda$. In fact, it is easy to see that they are nonincreasing from zero, as the lowest eigenvalue of $-\frac{1}{2} \Delta+V$ is negative. Next, again by an argument already used in the HF case, we obtain that $I_{\lambda}^{T F}$ and $I_{\lambda}^{T F W}$ are strictly decreasing when $\lambda \leq Z$, which amounts to saying that a strict inequality of type (3.10) then holds for both models. Indeed, if the mass $\int_{\mathbb{R}^{3}} \rho$ of a weak limit of a minimizing sequence is strictly less than $Z$, the operator

$$
-\frac{1}{2} \Delta+\frac{\int_{\mathbf{R}^{3}} \rho-Z}{|x|}
$$

has at least one negative eigenvalue and a contradiction is reached.

From then on, the TF case and the TFW case differ. In the former, one shows that for $\lambda>Z$ no minimizer exists. For the latter, one shows that for $\lambda=Z$ the Lagrange multiplier associated to the unique minimizer is strictly positive, which in turn implies that there still exists a minimizer for $\lambda$ slightly larger than $Z$. Indeed, the Lagrange multiplier can be recast as the first derivative of the infimum (here $I_{\lambda}^{T F W}$ ) with respect to the constraint (here the parameter of mass $\lambda$ ). In the language of chemistry, this justifies the name chemical potential for the Lagrange multiplier. This interpretation is analogous to that stating, in the theory of microeconomics, that the Lagrange multiplier is equal to the price. Formally (but this argument may be justified rigorously), we have here

$$
\frac{d}{d \lambda} I_{\lambda}^{T F W}=\frac{d}{d \lambda} E^{T F W}\left(\rho_{\lambda}\right)=-\theta_{\lambda}
$$

where $\rho_{\lambda}$ is the minimizer and $\theta_{\lambda}$ the associated Lagrange multiplier. Thus the positivity of $\theta_{\lambda}$ amounts to the strict monotonicity (here decreasing) of the infimum, and thus to the compactness of the minimization problem. Finally one proves the existence of $\lambda_{c}$ above which no minimizer exists and gets $\lambda_{c}>Z$. This is interpreted as saying that the TFW therefore allows for the existence of some negative ions, and not too negative ions, thereby agreeing with an expected physical property. 
3.2.3. The Euler-Lagrange equations. As may easily be seen, the Euler-Lagrange equation for the TF model reads

$$
\frac{5}{3} C_{T F} \rho^{2 / 3}=\left(-V-\rho \star \frac{1}{|x|}-\theta\right)_{+},
$$

where $\theta$ is the Lagrange multiplier. By examining the behaviour at infinity of the right-hand side, we see that $\theta \geq 0$ (otherwise $\rho \notin L^{1}\left(\mathbb{R}^{3}\right)$ ), which confirms that $I_{\lambda}^{T F}$ is nonincreasing with respect to $\lambda$. In addition, it can be shown that the critical value $\lambda_{c}$ for which $I_{\lambda}^{T F}$ ceases to be strictly decreasing with respect to $\lambda$ corresponds to $\theta=0$, and is indeed $\lambda_{c}=Z$.

It is worth mentioning that a useful rewriting of the above Euler-Lagrange equation is obtained by setting

$$
\Phi=-V-\rho \star \frac{1}{|x|}-\theta
$$

thus

$$
-\frac{1}{4 \pi} \Delta \Phi+\left(\frac{3}{5 C_{T F}} \Phi_{+}\right)^{3 / 2}=\sum_{k=1}^{M} \delta\left(\cdot-\bar{x}_{k}\right) .
$$

This equation is a nonlinear elliptic equation, whose treatment is easy for the function $t \longrightarrow\left(t_{+}\right)^{3 / 2}$ is nondecreasing.

Exchanging the role of $u=\sqrt{\rho}$ and $\Phi$, the mathematical nature of equation (3.56) has some similarity with that of the Euler-Lagrange equation for the TFW model, which reads

$$
-C_{W} \Delta u+V u+\frac{5}{3} C_{T F} u^{7 / 3}+\left(\rho \star \frac{1}{|x|}\right) u=-\theta u,
$$

for some $\theta$ that can be shown to be nonnegative (by the same monotonicity property of the infimum with respect to the constraint as above). Note that (3.56) is an equation with a right-hand side, while (3.57) is an eigenequation, though. For $\lambda<Z$, it can also be seen that $\theta>0$, either by using a variational argument analogous to that used in the Hartree-Fock case to prove (3.10), or by examining the behaviour at infinity of the potential and using an argument from spectral theory. Indeed, loosely speaking (but this can be made rigorous in particular by arguing on the spherical average), the potential

$$
W=V+\frac{5}{3} C_{T F} \rho^{2 / 3}+\rho \star \frac{1}{|x|}
$$

behaves as

$$
\frac{-Z+\lambda}{|x|}+\frac{5}{3} C_{T F} \rho^{2 / 3}
$$

at infinity, and thus for $\lambda<Z$, as $-a /|x|$ for some $a>0$. Now operators of the form $-\Delta-a /|x|$ have no eigenfunctions with zero energy.

On the other hand, when $\lambda=Z$, it can still be proven that $\theta>0$, but then no simple argument of spectral theory provides a proof. The result is indeed provided by a straightforward application of a lemma by B. Simon (see [159]): if $\varphi>0$ satisfies $-\Delta \varphi+W \varphi \geq 0$ outside a ball in $\mathbb{R}^{3}$, where $(\bar{W})_{+}$, the nonnegative part of the radial average $\bar{W}$ of $W$, is in $L^{3 / 2}$, then necessarily $\varphi \notin L^{2}$. Note that the result is restricted to signed eigenfunctions. 
From equation (3.57) also follow a lot of qualitative properties of $u=\sqrt{\rho}$ such as the regularity, and the asymptotic behaviour at the point nuclei and at infinity. All these properties reproduce those of the exact wavefunction.

Some symmetry can also be inferred from the equation, because of the uniqueness of the solution, a property in turn due to the strict convexity of the operator in the left-hand side: e.g., the solution is radially symmetric for an atom (i.e. in the case there is only one nucleus at the origin).

There is a large body of literature devoted to Thomas-Fermi-like models. We have outlined here only what we believe to be the most illustrative results, and we refer e.g. to [15, 16, 189, 190, 142, 160, 154, 151 for some other instances of mathematical works on this theory.

We conclude here this rapid tour of the basic results on the Thomas-Fermi and Thomas-Fermi-von Weizsäcker models by emphasizing that Thomas-Fermilike models have the twofold advantage of being good test theories both from the viewpoint of theoretical physics and from the viewpoint of mathematics. This is especially true for the TFW model which, in addition to the fact that it gives better numerical results, improves the TF model in many qualitative respects: see in particular the existence of negative ions, the properties of the ground state we mentioned above, and the existence of stable molecular systems we will prove below (although we shall also see in Section 4 that the latter existence occurs for some "bad" reason). Asymptotics of these models as the number of electrons grows to infinity will also be briefly mentioned below and will confirm that these theories are asymptotically exact, as indeed expected for theories based upon a comparison of the molecular system with a gas of electrons.

Even from the numerical viewpoint, algorithms can be advantageously tested on these models before being adapted to some more relevant cases.

3.3. A short description of the numerical strategies and challenges. In order to illustrate the difficulties encountered in the numerical simulation, we shall argue on the Hartree-Fock model, either in its energy form (3.7) or in its operator form (Euler-Lagrange equations (3.24) or (3.25)-(3.26)). It should be remarked that Kohn-Sham-like problems (3.49) indeed lead to the same kind of minimization procedure, and the same kind of solution procedure for the Euler-Lagrange equations, up to differences that can be considered as minor in such an expository survey mainly devoted to theoretical issues. The same is true for multiconfiguration methods or other more sophisticated methods. We therefore consider the Hartree-Fock model as illustrative enough to give a flavour of the generic numerical difficulties in computational quantum chemistry.

Regarding the numerics, the task of finding the electronic structure associated to a given configuration of nuclei clamped at positions $\bar{x}_{k}$ can be brought down to three main issues:

- how to discretize the variational space; i.e. how to find good basis functions $\chi_{k}$ for the expansion of the monoelectronic wavefunctions $\phi_{i}$;

- how to compute (assemble), for a given $N$-tuple $\left(\phi_{1}, \ldots, \phi_{N}\right)$, the selfconsistent operator (3.26) associated to the energy, or alternatively compute the various terms of the energy (3.6);

- how to attack the minimization problem (3.7), directly or by the solution procedure for its Euler-Lagrange equation (3.24) (or (3.25)-(3.26) ). 
The first two questions are intimately related. Historically, this is a clever remark by Boys [41] on an adequate choice of basis functions that changed the whole landscape of computational chemistry: Gaussians functions $\chi_{k}$ are well adapted to the problems for they allow for a rapid calculation of each of the multidimensional integrals

$$
\int_{\mathbb{R}^{3}} \int_{\mathbb{R}^{3}} \frac{\chi_{i}(x) \chi_{j}(x) \chi_{k}\left(x^{\prime}\right) \chi_{l}\left(x^{\prime}\right)}{\left|x-x^{\prime}\right|} d x d x^{\prime}
$$

arising in the electronic interaction terms of (3.7) and (3.26). Even with this simplification, it remains that the computation of the huge number of such integrals, $N^{4}$ in the whole generality, is a bottleneck for the whole computation. In fact, a significantly lower complexity, namely $N^{2}$ to $N^{3}$, is typically observed at this stage (because two nuclei in a large molecule generically are far away from one another). In fact the complexity measured in terms of the size of the physical system and that measured in terms of the dimension of the discretization space can differ, but the figures given here are vague enough to be valid in either case.

Some works have appeared in the chemistry literature that try to assess the validity of the various finite-dimensional approximations of the variational spaces, and to evaluate the rate of convergence of the approximation with respect to the size of the basis, see e.g. [135]. A rigorous systematic study of such questions appeared in 172 .

Once the variational space together with the strategy for the calculations of the terms of the energy (or of its first derivative) is fixed, the minimization is attacked. In almost all cases, this is done not by a direct minimization but rather by solving the Euler-Lagrange equation (here the Hartree-Fock equations), and this in spite of the fact that the two are not equivalent theoretically.

As the Hartree-Fock equations are self-consistent (the Fock operator (3.26) depends on the current iterate), the point there is to understand how to iterate on the nonlinearity, as naive fixed point strategies

$$
F_{\Phi^{n}} \phi_{i}^{n+1}=\lambda_{i}^{n+1} \phi_{i}^{n+1}
$$

of course do not work properly.

The chemistry literature abounds in articles describing convergence failures and recipes to avoid or cure them for the standard iteration procedures [186, 182]: see e.g. 137 193, 194, 195. The first attempt to understand mathematically the algorithms of computational chemistry dates back to [3], but a complete understanding of most of the algorithms commonly used appeared only recently [53, 50]. Then follows the construction of a new class of very efficient algorithms [139, based upon the combination of standard ideas of the chemical literature and ideas issued from mathematical considerations [54, 49]. The most efficient algorithms to date do not attack the minimization problem directly, but rather solve the Euler-Lagrange equations, while keeping track, in a weak sense, of some minimization paradigm: one approximates a minimizer and not a critical point (and this is precisely where the approach is revealed to be more efficient than others).

The very internal step of the above iteration procedure is to understand how to solve the linear problem: the Fock operator $F_{\Phi^{n}}$ being fixed at iteration $n$, its lowest $N$ eigenfunctions need to be determined. This requires the complete diagonalization of the operator. Indeed, e.g. for Gaussian basis functions, the size of the discrete variational space is typically twice the number of electrons, so determining the lowest $N$ eigenfunctions is of the same order of complexity as finding all of them. A 
standard diagonalization procedure is thus used (note that one of the most famous algorithms for this purpose is, in its earliest form, due to a chemist, Davidson). Therefore a complexity of $N^{3}$ is expected.

Globally, computing the Hartree-Fock ground state typically requires between $N^{3}$ and $N^{4}$ operations. Using post-Hartree-Fock methods such as MCSCF can even go up to $N^{8}$ operations or more. It is now easy to measure why only small systems can be attacked by so many precise ab initio methods.

Less precise models, called parameterized models, can of course be resorted to, but on the other hand huge efforts have been devoted in the last two decades to reducing the complexity of $a b$ initio methods down to a linear complexity.

All the steps above need special attention. The assembling step may employ fast multiple methods to evaluate multidimensional integrals for electrostatic interactions. The nonlinear iterations speed up with the help of quadratically convergent algorithms. Finally, the inner loop consisting in the solution for the linear problem is approached by dedicated techniques. Such techniques are essentially based upon the following observation: the diagonalization is not stricto sensu needed: only the determination of the projector on the lowest $N$ eigenfunctions is required. This can easily be seen by restating the Hartree-Fock problem in the language of density matrices (a language that is indeed very efficient for numerical analysis, and for other purposes that will be seen in Section 6). Introducing

$$
\mathcal{D}=\sum_{i=1}^{N}\left(\phi_{i}, \cdot\right)_{L^{2}} \phi_{i}
$$

the HF problem reads

$$
\inf \left\{\operatorname{Trace}(h \mathcal{D})+\frac{1}{2} \operatorname{Trace}(\mathcal{G}(\mathcal{D}) \cdot \mathcal{D}), \quad \mathcal{D}^{2}=\mathcal{D}=\mathcal{D}^{*}, \quad \operatorname{Trace}(\mathcal{D})=N\right\}
$$

with $h=-\frac{1}{2} \Delta+V$ and

$$
(\mathcal{G}(\mathcal{D}) \cdot \phi)(x)=\left(\rho_{\mathcal{D}} \star \frac{1}{|y|}\right)(x) \phi(x)-\int_{\mathbf{R}^{3}} \frac{\tau_{\mathcal{D}}\left(x, x^{\prime}\right)}{|x-y|} \phi\left(x^{\prime}\right) d x^{\prime} .
$$

Such linear scaling methods, overviewed e.g. in 119, 117, 188, still need improvements (we do not go into details, but only mention that there are cases of outstanding interest when most of them fail in doing better than say an $N^{2}$ complexity). The future of computational quantum chemistry is likely to depend on the success of such methods and their ability to attack large systems.

3.4. Beyond the simple setting of the isolated molecule at zero temperature. The above modelling issues have been exposed for the simplest possible situation, namely that of an isolated molecular system at zero temperature. Of course, in practice, computational chemistry consists of simulating the behaviour of real systems in situ, or in other words the response of the systems to various solicitations.

3.4.1. Positive temperature effects. The first issue of interest is that of temperature. Contrary to the $0^{\circ} \mathrm{K}$ setting, where the electrons are frozen in the lowest eigenstates of the Hamiltonian, a positive temperature generates a dispersion of the various electrons amongst all energy levels. The above picture is standard in a linear setting, but is also valid for the nonlinear problems at hand. As an instance of such 
a setting, we now mention the Hartree-Fock model with temperature that reads (see [164])

$$
\begin{aligned}
\inf \left\{\frac{1}{2} \int_{\mathbb{R}^{3}} \sum_{i=1}^{+\infty} n_{i}\left|\nabla \phi_{i}\right|^{2}+\right. & \int_{\mathbf{R}^{3}}\left(V+V_{c}\right) \rho+\frac{1}{2} \int_{\mathbb{R}^{3}} \int_{\mathbf{R}^{3}} \frac{\rho(x) \rho\left(x^{\prime}\right)}{\left|x-x^{\prime}\right|} d x d x^{\prime} \\
& -\frac{1}{2} \int_{\mathbf{R}^{3}} \int_{\mathbf{R}^{3}} \frac{\left|\rho\left(x, x^{\prime}\right)\right|^{2}}{\left|x-x^{\prime}\right|} d x d x^{\prime}+\frac{1}{\beta} S(n) ; \\
0 \leq & \left.n_{i} \leq 1, \quad \phi_{i} \in H^{1}, \quad \int_{\mathbb{R}^{3}} \phi_{i} \phi_{j}=\delta_{i j}, \quad \sum_{i=1}^{+\infty} n_{i}=N\right\},
\end{aligned}
$$

where we define $\rho\left(x, x^{\prime}\right)=\sum_{i=1}^{+\infty} n_{i} \phi_{i}(x) \phi_{i}\left(x^{\prime}\right), \rho(x)=\sum_{i=1}^{+\infty} n_{i}\left|\phi_{i}(x)\right|^{2}$, and

$$
S(n)=\sum_{i=1}^{+\infty}\left[n_{i} \log \left(n_{i}\right)+\left(1-n_{i}\right) \log \left(1-n_{i}\right)\right]
$$

The potential $V_{c}$ is a confinement potential, say $V_{c}(x)=|x|^{2}$ to fix the ideas. The $n_{i} \in[0,1]$ are called occupation numbers and translate the fact that at positive temperatures, eigenstates can be partially occupied, contrary to the case of zero temperature where $n_{i} \in\{0,1\}$. The constant $\beta$ is the Boltzmann constant related to the temperature $T$ and the Planck constant $k$ by $\beta=\frac{1}{k T}$. Regarding the term (3.61), it models the entropy of the system.

It is proven in 164 that there exists a minimizer for this problem, and that this minimizer satisfies, up to a permutation of the indices and up to an orthogonal transform, the Euler-Lagrange equations

$$
\left\{\begin{array}{l}
-\frac{1}{2} \Delta \phi_{i}+\left(V+V_{c}+\rho \star \frac{1}{|x|}\right) \phi_{i}-\int_{\mathbb{R}^{3}} \frac{\rho\left(x, x^{\prime}\right) \phi_{i}\left(x^{\prime}\right)}{\left|x-x^{\prime}\right|} d x^{\prime}+\theta_{i} \phi_{i}=0, \\
n_{i}=\frac{\mu e^{\beta \theta_{i}}}{1+\mu e^{\beta \theta_{i}}} .
\end{array}\right.
$$

In the above equations, the $\theta_{i}$ form, as in the zero temperature case, the diagonalized matrix of Lagrange multipliers for the orthonormality constraints. On the other hand, $\mu$ is the Lagrange multiplier of the constraint $\sum_{i=1}^{+\infty} n_{i}=N$.

It is interesting to look at the $\beta \longrightarrow+\infty$ limit in order to see whether the zero temperature case is recovered. It can be proven that $\mu$ behaves like $e^{-\beta \bar{\theta}}$ for some $\bar{\theta}$, and thus that either $\theta_{i}>\bar{\theta}$ in which case $n_{i} \longrightarrow 1$, or $\theta_{i} \leq \bar{\theta}$ in which case $n_{i} \longrightarrow 0$. The former occurs for $N$ values of the indices $i$, and we therefore indeed recover the standard Hartree-Fock case.

Apart from this result on the Hartree-Fock model with temperature, we are aware of no other results on positive temperature models. A careful mathematical examination of the most commonly used models could be of great interest.

Remark 3.12. Implicitly, the modelling of finite temperature effects implies the understanding of the notion of excited states in the model under consideration. On purpose we have focused on the electronic ground state. The very definition of 
excited states for the nonlinear approximations of the Schrödinger model manipulated here is unclear. We refer to [150] for a promising approach, making use of the MCSCF model introduced above in Section 3.1.5.

3.4.2. Molecules under electromagnetic fields. Another situation of interest is that where the molecular system is subjected to an electromagnetic field. The effect of the electric field on the one hand, and that of the magnetic field on the other hand, are of a quite different mathematical nature.

Electrons experiencing an electric field can escape from the attraction of the nuclei, and therefore the presence of an electric field is a serious difficulty when examining the variational problem. One may measure the difficulty simply by adding a term

$$
\int_{\mathbb{R}^{3}} E x_{1} \rho(x) d x
$$

to (e.g.) the TFW energy functional (3.53). In (3.63), $E$ denotes a constant value of the electric field, here aligned with the $x_{1}$ axis (first component of $x \in \mathbb{R}^{3}$ ). The electric field is then treated in a perturbation setting. It is immediately seen, by pushing some mass to infinity in the direction $x_{1} \longrightarrow-\infty$ (say $E>0$ to fix the ideas), that the corresponding minimization problem is not well posed. An analysis is provided in [51]. It must be emphasized that, for such nonlinear variational problems, it is not well understood how to deal with electric fields in a static setting (the resonance theory, e.g. exposed in [185, that is the standard tool of the linear setting is not so clearly developed here). However, time-dependent problems with electric fields (such as those issued from laser control applications, see Remark 5.1) can be posed on a rigorous mathematical ground.

On the other hand, the primary mathematical effect of a magnetic field is not to create a possible loss of compactness in the minimization problem, but rather to complicate the models themselves, through the need to reintroduce the spin variables. Examples of mathematical works dealing with some variants of ThomasFermi-type theories in the presence of a magnetic field are [18, 19, 121, 122, 144].

3.4.3. Condensed phases. There is another effect we would like to point out. Even with the above modifications, the modelling explained so far concerns molecular systems in the gas phase. Indeed, the fact that the system is often included in a condensed phase has not been accounted for.

There again, the situation highly differs, depending upon whether the condensed phase is a liquid one or a solid one.

The standard modelling of the liquid phase is based upon ideas introduced by Onsager in the 1930s. The liquid environment of the molecular system under study is treated as a continuum dielectric. The continuum dielectric is assumed to fill in the whole space except for some cavity, $\Omega$, enclosing the molecular system. The effect of this continuum is to modify the interaction law (Coulombic in the gas phase) between charged particles in the molecular system. Mathematically, the $\frac{1}{4 \pi|x|}$ interaction law, which is the elementary solution (or the Green function) of the Laplacian operator in the whole space $\mathbb{R}^{3}$, is replaced by the solution $G$ of

$$
-\operatorname{div}(\epsilon(x) \nabla G)=\delta,
$$


where the permittivity $\epsilon$ is a space-dependent scalar (to simplify)

$$
\epsilon(x)=\left\{\begin{array}{lll}
\epsilon_{0}=\frac{1}{4 \pi}, & \text { constant permittivity of the vaccuum, } & \text { when } x \in \Omega, \\
\epsilon_{1} \neq \epsilon_{0}, & \text { constant permittivity of the solvent, } & \text { when } x \notin \Omega .
\end{array}\right.
$$

The terms of electrostatic interactions in the energy functional are modified correspondingly. The kinetic energy term is kept unchanged. In spite of the fact that no systematic study of such models has appeared in the literature, it is believed that a given model (say the HF model or the TFW model) enjoys the same mathematical properties in a vacuum and in the liquid phase modelled as above. The reason for such an intuition is that what basically governs the mathematical properties of these variational problems is the long range behaviour of the interaction potential: now the behaviour of the solution $G$ to (3.64) at infinity is (up to a multiplicative constant) like that of the Coulomb potential. On the other hand, it should be mentioned that from the computational viewpoint, models for the liquid phase have motivated a long series of works: see [56] for a survey of the numerical approach, and Chapter 3 of [48] for the consequences on computations of real cases.

Regarding the modelling of the solid phase, a lot of questions of prominent mathematical interest arise. We shall devote the entire Section [6 below to this setting. The reader will see that again, the modelling is based upon a convenient modification of the interaction law between charged particles. In the liquid case, one representative molecular system (the solvated molecule) is embedded in a continuum (the solvent), the latter being modelled at a macroscopic level. Here, the whole infinite domain $\mathbb{R}^{3}$ is described microscopically (there is a periodic repetition of the same molecular structure), and consequently the microscopic variational problem on which we focus is considerably complicated.

3.5. Back to the Schrödinger equation. Is is instructive at this stage to come back to the electronic Schrödinger equation that we have introduced in (2.2) as the original model and have subsequently approximated. We would like to briefly report on the investigations along the following lines: what type of relation, on the mathematical level, can be proven to exist between the original model and its approximations?

As the Thomas-Fermi theory originates from the modelling of electron gases, it is expected that the regime where it is the closest to the Schrödinger model is that when the number of electrons is large. The relation between the ThomasFermi theory and the Schrödinger problem is indeed often investigated for the case of a heavy atom in the limit where the nuclear charge $Z$ goes to infinity and the number of electrons $N$ is proportional to $Z$. In this limit the Thomas-Fermi theory is asymptotically exact, in the sense that it gives the leading order for the expansion of the ground-state energy $E_{Z}$ provided by the Schrödinger approximation. More precisely, we have

$$
E_{Z}=-c_{0} Z^{7 / 3}+c_{1} Z^{2}-\frac{10}{9} c_{2} Z^{5 / 3}+o\left(Z^{5 / 3}\right) .
$$

The first term $-c_{0} Z^{7 / 3}$ is equal to the Thomas-Fermi energy. The second and third terms are corrections to it that were initially conjectured as being $c_{1} Z^{2}-c_{2} Z^{5 / 3}$ (for precise values of $c_{1}, c_{2}$ ) by Scott in the 1950s. Next the constant in the third 
term was corrected with the $\frac{10}{9}$ factor in a new conjecture by Schwinger. Fefferman and Seco established rigorously the expansion (3.66) in [106, 107, 108].

Another way of examining the $Z \longrightarrow+\infty$ limit would be to argue on the crystal case. In that case, the nuclear charge also becomes infinite, but instead of concentrating at a given point, as is the case for a heavy atom, it spreads in a homogeneous way all over the space. Finding to which extent the ground-state energy $I\left(\Lambda_{N}\right)$ (in the setting of Thomas-Fermi-like models) of a system converging to a crystal asymptotically deviates from the same energy at the Schrödinger level could advantageously complement the understanding of the relation between models. To our knowledge, such a study has not been performed to date.

In the vein of the results known for the Thomas-Fermi model, V. Bach proved in [5 that the Hartree-Fock ground-state energy is also asymptotically exact in the limit of a heavy atom.

Remark 3.13. An alternative strategy, in order to assess the validity of both the Hartree-Fock approximation and the Density Functional type theories, is to come back to the Schrödinger equation and construct a hierarchy of equations, with the density matrices at all orders. This strategy is in some sense dictated by an emerging class of methods in computational chemistry. Indeed, apart from the wavefunctions and DFT-type methods, a third class of approximation methods, less utilized though, consists of manipulating density matrices instead of wavefunctions or densities. This approximation consists of noticing that, since the Hamiltonian only contains one- and two-electron operators, the energy $\left\langle\psi_{e}, H_{e} \psi_{e}\right\rangle$ can be expressed in terms of the two-particle density matrix

$$
\begin{aligned}
& \gamma_{2}\left(x_{1}, x_{2}, x_{1}^{\prime}, x_{2}^{\prime}\right) \\
& \quad=\int_{\mathbb{R}^{3(N-2)}} \psi_{e}\left(x_{1}, x_{2}, x_{3}, \ldots, x_{N}\right) \psi_{e}\left(x_{1}^{\prime}, x_{2}^{\prime}, x_{3}, \ldots, x_{N}\right) d x_{3} \cdots d x_{N} .
\end{aligned}
$$

Contrary to the DFT setting, the explicit expression of the energy is then known, and the minimum of the energy as a functional of $\gamma_{2}$ can be sought. However, this must be done under the restriction that $\gamma_{2}$ is derivable from an (antisymmetric) $N$-electron wave function $\psi_{e}$. This restriction is known as an $N$-representability problem and is one of the unsolved problems of theoretical chemistry. The current state of the art of chemistry is to circumvent this difficulty by rather considering the stationarity conditions with respect to variations of the wavefunction, namely the Schrödinger equation, and then formulating it in terms of density matrices. One can formally start with the Schrödinger equation

$$
H_{e} \psi_{e}=E_{e} \psi_{e},
$$

multiply it by $\psi_{e}$ and integrate over a subset $\left(x_{p+1}, \ldots, x_{N}\right)$ of the variables $\left(x_{1}, \ldots\right.$, $x_{N}$ ). A hierarchy of equations is obtained, that involves the reduced matrices $\gamma_{p}$ of different ranks, i.e. the matrices built in the same fashion as (3.67) with integration over $p$ of the $x_{i}$. Recent works in the chemistry literature have shown how to construct approximations of the $\gamma_{p}$ in terms of $\gamma_{k}$ for all $k<p$, and to define a hierarchy of contracted Schrödinger equations that can be truncated at any particle rank $p$. This is a lively topic of theoretical chemistry, very promising in a computational perspective; see 73 . Notice that we shall see a time-dependent version of the above approach in Section 5 below. 


\section{Geometry optimization: From atoms to molecules}

Section 3 has only addressed the problem of finding the electronic state of the molecular system while considering the nuclei as parameters, i.e. placed at fixed positions known in advance. We now turn to the problem of finding the optimal positions (one speaks of a configuration) of the nuclei so that the energy of the whole system, electrons and nuclei, is minimized.

Let us begin (for a change) with the Thomas-Fermi-like models and introduce the following notation. In order to emphasize the role played by the positions $\bar{x}_{k}$ and the charges $z_{k}$ of the $M$ nuclei, we denote by $I_{\lambda}\left(z_{1}, \ldots, z_{M} ; \bar{x}_{1}, \ldots, \bar{x}_{M}\right)$ the electronic minimization problems we used to denote by $I_{\lambda}^{T F}\left(\bar{x}_{1}, \ldots, \bar{x}_{M}\right), I_{\lambda}^{T F W}\left(\bar{x}_{1}, \ldots, \bar{x}_{M}\right)$, $I_{\lambda}^{T F D}\left(\bar{x}_{1}, \ldots, \bar{x}_{M}\right), I_{\lambda}^{T F D W}\left(\bar{x}_{1}, \ldots, \bar{x}_{M}\right)$ above, depending on the theory under study. Then we set

$$
I\left(\lambda ; z_{1}, \ldots, z_{M}\right)=\inf _{\left(\bar{x}_{1}, \ldots, \bar{x}_{M}\right) \in\left(\mathbf{R}^{3}\right)^{M}} I_{\lambda}\left(z_{1}, \ldots, z_{M} ; \bar{x}_{1}, \ldots, \bar{x}_{M}\right)+\sum_{1 \leq k \neq k^{\prime} \leq M} \frac{z_{k} z_{k^{\prime}}}{\left|\bar{x}_{k}-\bar{x}_{k^{\prime}}\right|} .
$$

Note that the last term accounting for the repulsion between the nuclei of the molecule is added to the electronic energy so that the complete energy of the system will be manipulated.

Definition 4.1. We will say that the problem is well-posed if any minimizing sequence $\left(\bar{x}_{1}^{n}, \ldots, \bar{x}_{M}^{n}\right)$ of (4.1) is relatively compact in $\left(\mathbb{R}^{3}\right)^{M}$ up to a translation, i.e. there exists some sequence $y_{n} \in \mathbb{R}^{3}$ such that $\bar{x}_{k}^{n}+y_{n}$ is relatively compact in $\mathbb{R}^{3}$ for all $1 \leq k \leq M$.

Of course, the restriction of compactness up to a translation is necessary since problem $I_{\lambda}\left(z_{1}, \ldots, z_{M} ; \bar{x}_{1}, \ldots, \bar{x}_{M}\right)$ is obviously invariant under a global translation of the positions of the nuclei. Under the additional assumptions that enforce the compactness of the electronic problem, the above well-posedness property implies the relative compactness of the global minimizing sequence (positions of nuclei, wavefunction). For instance, if $\lambda \leq \lambda_{c}$ in the TFW model, then we know that any minimizing sequence $\left(\bar{x}_{1}^{n}, \ldots, \bar{x}_{M}^{n}, \sqrt{\rho^{n}}\right)$ is compact in $\left(\mathbb{R}^{3}\right)^{M} \times H^{1}\left(\mathbb{R}^{3}\right)$, up to a translation on each $\bar{x}_{k}$.

Unlike the translation invariance of the problem, which is in some sense an obstruction to compactness, the second geometric invariance of the problem

$$
I_{\lambda}\left(z_{1}, \ldots, z_{M} ; \bar{x}_{1}, \ldots, \bar{x}_{M}\right)
$$

namely the fact that we may globally rotate the set of positions $\left(\bar{x}_{1}, \ldots, \bar{x}_{M}\right)$, will turn out to be an advantage that will help in showing the well-posedness of problem (4.1). The reason is twofold: on the one hand, unlike the translation group, the rotation group is compact, thus has no impact on the existence of a minimizer for minimization problems, and on the other hand, the rotational invariance allows one to conduct arguments in terms of spherical averages, for which most Coulomb-like interactions can be evaluated explicitly.

4.1. Statement of the main results. The results known to date for the optimization problem for TF-type models are now summarized.

Theorem 4.2. 66 67 67 68 69 , 69timization geometry for TF-type models 
- (1) The problem is well-posed if the following strict subadditivity condition,

$$
I\left(\lambda ; z_{1}, \ldots, z_{M}\right)<I\left(\lambda_{1} ; z_{i_{1}}, \ldots, z_{i_{p}}\right)+I\left(\lambda_{2} ; z_{i_{p+1}}, \ldots, z_{i_{M}}\right),
$$

holds for any nontrivial partition $\left(\lambda_{1} ; z_{i_{1}}, \ldots, z_{i_{p}}\right)-\left(\lambda_{2} ; z_{i_{p+1}}, \ldots, z_{i_{M}}\right)$ of $\left(\lambda, z_{1}, \ldots, z_{M}\right)$ with $\lambda_{1}+\lambda_{2}=\lambda,\left\{i_{1}, \ldots, i_{M}\right\}=\{1, \ldots, M\}$. Whenever (4.2) holds, we say that systems $\mathcal{S}_{1}=\left(\lambda_{1} ; z_{i_{1}}, \ldots, z_{i_{p}}\right)$ and $\mathcal{S}_{2}=\left(\lambda_{2} ; z_{i_{p+1}}, \ldots, z_{i_{M}}\right)$ can be bound.

- (2) We assume $Z \geq \lambda$. The problem is well-posed if and only if condition (4.2) holds for any nontrivial partition such that $Z_{1}=z_{i_{1}}+\ldots+z_{i_{p}} \geq \lambda_{1}$, $Z_{2}=z_{i_{p+1}}+\ldots+z_{i_{M}} \geq \lambda_{2}$ (dissociation into positively charged subsystems), and the minimizers of $I\left(\lambda_{1} ; z_{i_{1}}, \ldots, z_{i_{p}}\right)$ and $I\left(\lambda_{2} ; z_{i_{p+1}}, \ldots, z_{i_{M}}\right)$ share the same Lagrange multiplier (same chemical potential in terms of chemistry).

- (3) We assume $Z=\lambda$. The problem is well-posed if and only if condition (4.2) holds for any nontrivial partition such that $z_{i_{1}}+\ldots+z_{i_{p}}=\lambda_{1}$, $z_{i_{p+1}}+\ldots+z_{i_{M}}=\lambda_{2}$ (dissociation into neutral subsystems), and the minimizers of $I\left(\lambda_{1} ; z_{i_{1}}, \ldots, z_{i_{p}}\right)$ and $I\left(\lambda_{2} ; z_{i_{p+1}}, \ldots, z_{i_{M}}\right)$ share the same Lagrange multiplier.

- (4) In the TFW and TFDW cases, for $Z=\lambda$ the problem is well-posed.

It is known since a work by Teller (see 159 for a complete mathematical argument) that in the TF case, two neutral systems $\mathcal{S}_{1}$ and $\mathcal{S}_{2}$ (i.e. with $z_{i_{1}}+\ldots+z_{i_{p}}=\lambda_{1}$ and $z_{i_{p+1}}+\ldots+z_{i_{M}}=\lambda_{2}$ ) can never be bound; i.e. (4.2) cannot hold. This negative result, known as Teller's no binding Theorem, confirms that in many respects the TF theory is not qualitatively correct. The key mathematical ingredient of Teller's Theorem is a property of monotonicity of the effective potential $\Phi$ defined by (3.55) with respect to the set of nuclei in the system. Suppose indeed that we are given two potentials $V_{1}$ and $V_{2}$, such that $-\Delta V_{1} \geq-\Delta V_{2}$, a fact which occurs e.g. when $V_{2}=V_{1}-\frac{z_{M+1}}{\left|\cdot-\bar{x}_{M+1}\right|}$ (imagine an $(M+1)$-th nucleus, and the corresponding number of electrons, is being added to a molecule already composed of $M$ nuclei and $M$ electrons). Then define $\Phi_{1}$ and $\Phi_{2}$, from $V_{1}$ and $V_{2}$ respectively, by (3.55) with the same $\theta$ (which occurs when the two systems are neutral, since $\theta=0$ for both). Simply by considering the set $\left\{x / \Phi_{1}(x)>\Phi_{2}(x)\right\}$, one remarks that

$$
-\frac{1}{4 \pi} \Delta\left(\Phi_{1}-\Phi_{2}\right)=\frac{1}{4 \pi}\left(\Delta V_{1}-\Delta V_{2}\right)-\left(\frac{3}{5 C_{T F}}\right)^{3 / 2}\left(\left(\Phi_{1}\right)_{+}^{3 / 2}-\left(\Phi_{2}\right)_{+}^{3 / 2}\right) \leq 0
$$

on this set, which shows by the maximum principle that it is empty. It follows that $\Phi_{1} \leq \Phi_{2}$ in this situation. The potential $\Phi$ therefore increases when the molecule grows. In turn, this property leads to Teller's Theorem.

Remark 4.3. This property of monotonicity of $\Phi$ also plays a crucial role in the thermodynamic limit of the TF model. The thermodynamic limit problem will be addressed in Section 6. Schematically, the number of nuclei is increased, until an infinite crystal of nuclei is built, and the limit of the corresponding electronic structure is examined. In the TF case, the limit of the potentials $\Phi$ is a monotone limit. It is a somehow paradoxical feature of the TF model that the same monotonicity property both forbids the binding of two atoms and allows the study of the bulk limit.

A series of works [66, 67, 68, 69] by I. Catto and one of us (PLL) settled in the 1990s the other instances of TF-like models and showed that the models containing 
the von Weiszäcker term present good qualitative behaviour: neutral systems do bind to form a stable molecular system. The above theorem is the main result along these lines. Previous results were established in [7] and [14] in the case $M=2$ and for particular values of the charges $z_{1}$ and $z_{2}$. On the basis of such preliminary results, E. Lieb conjectured in [159] that the binding of neutral systems should occur in the TFW model.

Remark 4.4. When the model is not convex, there might be an ambiguity in the statement of Assertions (2) and (3) as many minimizers of $I\left(\lambda_{1} ; z_{i_{1}}, \ldots, z_{i_{p}}\right)$ and $I\left(\lambda_{2} ; z_{i_{p+1}}, \ldots, z_{i_{M}}\right)$ might exist. It turns out that it suffices to consider those that share the same Lagrange multiplier, as they are the only ones that may be created in dissociations.

\section{Remark 4.5. On the uniqueness of the optimal configuration}

We emphasize that no result exists to date on the uniqueness, or nonuniqueness, of the optimal configuration of nuclei. It is known from experiment that many optimal configurations may exist for some systems, while others have a unique optimal configuration. No mathematical study has ever provided any insight on this issue.

In fact, the same question can be investigated in the settings of Hartree and Hartree-Fock models. The same mathematical tools can be applied and provide the following result.

\section{Theorem 4.6. 66, 67, 68, 69 Optimization geometry for $\mathbf{H}$ and HF models}

- Parts (1), (2), (3) of Theorem 4.2 above hold true for both the Hartree and Hartree-Fock models, with $N, N_{1}, N_{2}$ replacing $\lambda, \lambda_{1}, \lambda_{2}$ respectively. Note that by this notation convention, it is meant that only integer dissociations $N_{1} \in \mathbb{N}, N_{2} \in \mathbb{N}$ have to be considered.

- Part (4) is true for the Hartree model.

Remark 4.7. Part (4) is an open issue for the HF model.

The problem of geometry optimization for the Hartree-Fock problem is still open because it seems that the techniques applied to the other cases cannot accommodate with the orthonormality constraint. Even in the simple case of the hydrogen molecule $(M=2, N=2)$, no result exists.

The proof of Theorems 4.2 and 4.6 requires a lot of technical tools. Following the general spirit of this survey article, we will only outline the proof and the main arguments, moreover restricted to the Thomas-Fermi-von Weizsäcker model and to a diatomic system $(M=2)$. We will eventually briefly indicate the necessary modifications of the argument to deal with the other cases stated in the above theorems.

We begin by giving a short formal argument that explains the main lines of the proof. Suppose the system $\left\{\lambda, z_{1}, z_{2}\right\}$ consisting of $\lambda$ electrons and two nuclei of charge $z_{1}$ and $z_{2}$ respectively dissociates into a subsystem $\left\{\lambda_{1}, z_{1}\right\}$ and a second one $\left\{\lambda_{2}=\lambda-\lambda_{1}, z_{2}\right\}$ at distance $\Delta$ from the former. Then, the balance of electrostatic 
energy $E_{\text {elec }}$ reads, at order $1 / \Delta$,

$$
\begin{aligned}
E_{\text {elec }}\left(\left\{\lambda, z_{1}, z_{2}\right\}\right) & -E_{\text {elec }}\left(\left\{\lambda_{1}, z_{1}\right\} \cup\left\{\lambda_{2}, z_{2}\right\} \text { at distance } \Delta\right) \\
& \approx-\frac{z_{1} \lambda_{2}}{\Delta}-\frac{z_{2} \lambda_{1}}{\Delta}+\frac{\lambda_{1} \lambda_{2}}{\Delta}+\frac{z_{1} z_{2}}{\Delta} \\
& =\frac{\left(z_{1}-\lambda_{1}\right)\left(z_{2}-\lambda_{2}\right)}{\Delta} .
\end{aligned}
$$

Now, for a neutral system, $z_{1}+z_{2}=\lambda_{1}+\lambda_{2}$; thus $\left(z_{1}-\lambda_{1}\right)\left(z_{2}-\lambda_{2}\right) \leq 0$. It follows that if $z_{1} \neq \lambda_{1}$ and $z_{2} \neq \lambda_{2}$, the product, thus the balance (4.3), is negative, and no such separation can occur. Therefore, proving stability amounts to preventing separation into two neutral subsystems $z_{1}=\lambda_{1}$ and $z_{2}=\lambda_{2}$ (this is why (1) is equivalent to (2) or (3) in Theorem 4.2). For this purpose, one needs to study more precisely the balance. In fact, pushing forward the expansion (4.3) in powers of $1 / \Delta$ does not allow us to prove stability because all higher-order terms are null. It will be shown, by careful examination, that the balance indeed depends exponentially on $1 / \Delta$ and is negative, thus showing the stability.

In the diatomic case, we may assume, without loss of generality, that the first nucleus stands at $\bar{x}_{1}=0$ and the second one at $\bar{x}_{2}=\Delta \mathbf{e}$ where $\Delta$ is the interatomic distance and $\mathbf{e}$ is some fixed unitary vector.

Instead of (4.1) we may write (in the TFW case we deal with here)

$$
\begin{gathered}
I\left(\lambda, z_{1}, z_{2}\right)=\quad \inf _{\Delta} \inf \left\{E_{\infty}^{T F W}(\rho)-\int_{\mathbb{R}^{3}} \frac{z_{1}}{|x|} \rho-\int_{\mathbb{R}^{3}} \frac{z_{2}}{|x-\Delta \mathbf{e}|} \rho ;\right. \\
\left.\sqrt{\rho} \in H^{1}\left(\mathbb{R}^{3}\right), \quad \int_{\mathbb{R}^{3}} \rho=\lambda\right\}+\frac{z_{1} z_{2}}{\Delta}, \\
\text { notation } \inf _{\Delta} I_{\lambda}(\Delta)+\frac{z_{1} z_{2}}{\Delta},
\end{gathered}
$$

where $E_{\infty}^{T F W}(\rho)$ denotes the TFW energy functional with $V=0$ (the notation comes from the fact we have ejected all nuclei to infinity and remain with $V=0$ ).

4.2. Sketch of proof for the TFW model in the diatomic case. The first remark is that

- (i) pushing some mass at infinity as we did in the proof of the existence of a Hartree-Fock ground state, we may show that, in the language of (4.2), the subadditivity condition

$$
I\left(\lambda ; z_{1}, z_{2}\right) \leq I\left(\lambda_{1} ; z_{1}\right)+I\left(\lambda_{2} ; z_{2}\right)
$$

always holds;

- (ii) if equality holds in (4.5), then a simple argument (based upon the reconstruction of a minimizing sequence for $I\left(\lambda ; z_{1}, z_{2}\right)$ from minimizing sequences of $I\left(\lambda_{1} ; z_{1}\right)$ and $I\left(\lambda_{2} ; z_{2}\right)$ respectively) shows there exists a noncompact minimizing sequence, which shows the "only if" part of assertion (1) of Theorem 4.2

Next, we note that proving the existence of a minimizing $\Delta$ for

$$
\inf _{\Delta} I_{\lambda}(\Delta)+\frac{z_{1} z_{2}}{\Delta}
$$

amounts to proving that

$$
\inf _{\Delta} I_{\lambda}(\Delta)+\frac{z_{1} z_{2}}{\Delta}<\lim _{\Delta \longrightarrow+\infty} I_{\lambda}(\Delta) .
$$


Thus proving (1) of Theorem 4.2 for a diatomic system amounts to identifying the $\Delta \longrightarrow \infty$ limit as

$$
\lim _{\Delta \longrightarrow+\infty} I_{\lambda}(\Delta)=\inf _{\mu \in[0, \lambda]}\left(I_{\mu}\left(z_{1}\right)+I_{\lambda-\mu}\left(z_{2}\right)\right)
$$

where on the right-hand side $I_{\nu}(z)$ denotes the atomic problem with nuclear charge $z$ and $\nu$ electrons.

The proof of (4.7) falls into three steps. First it is easily shown that $\limsup _{\Delta \longrightarrow+\infty} I_{\lambda}(\Delta)$ $\leq$ r.h.s. by considering two compactly supported test functions that conveniently approach the infima $I_{\mu}\left(z_{1}\right)$ and $I_{\lambda-\mu}\left(z_{2}\right)$ respectively and placing them at a distance $\Delta$. An evaluation at order $1 / \Delta$ of the energy of the system obtained yields the bound from above.

The last two steps aim at showing the key point: $\liminf _{\Delta \longrightarrow+\infty} I_{\lambda}(\Delta) \geq$ r.h.s. First, we consider some sequence $\Delta_{n} \longrightarrow+\infty$ and the associated minimizer $\rho_{n}$ for each $n$ (for simplicity we restrict ourselves to the case when $\lambda \leq Z$ and thus all minimization problems with respect to the electronic density $\rho$ are attained; some technical modifications are needed to also establish assertion (1) of Theorem 4.2 for general $\lambda$ ). Denoting by $u_{n}=\sqrt{\rho_{n}}$, it is easy to see that $u_{n}$ is bounded in $H^{1}$, thus up to an extraction, converges to some $u$, weakly in $H^{1}$, strongly in $L^{p}$ locally for all $1 \leq p<+\infty$, and also almost everywhere. In fact, in this framework of locally compact problems, $u$ can be interpreted as the part of $u_{n}$ that remains at finite distance while $n \longrightarrow+\infty$. We then claim that

$$
\begin{aligned}
E_{\infty}\left(u_{n}^{2}\right) & -\int_{\mathbf{R}^{3}} \frac{z_{1}}{|x|} u_{n}^{2}-\int_{\mathbf{R}^{3}} \frac{z_{2}}{\left|x-\Delta_{n} \mathbf{e}\right|} u_{n}^{2} \\
= & \left.E_{\infty}\left(u^{2}\right)-\int_{\mathbf{R}^{3}} \frac{z_{1}}{|x|} u^{2}\right) \\
& +\left(E_{\infty}\left(\left(u_{n}-u\right)^{2}\right)-\int_{\mathbf{R}^{3}} \frac{z_{2}}{\left|x-\Delta_{n} \mathbf{e}\right|}\left(u_{n}-u\right)^{2}\right) \\
& +\epsilon_{n},
\end{aligned}
$$

with $\epsilon_{n} \longrightarrow 0$. In order to show this decomposition of the energy, one studies each term of the energy functional and shows that each term dissociates into the two expected parts. Quadratic terms (such as indeed the mass constraint $\int_{\mathbf{R}^{3}} u_{n}^{2}=\lambda$ ) are easy to deal with because

$$
\int_{\mathbf{R}^{3}} u_{n}^{2}-\int_{\mathbf{R}^{3}}\left(u_{n}-u\right)^{2}=\int_{\mathbf{R}^{3}} u\left(2 u_{n}-u\right) \longrightarrow \int_{\mathbf{R}^{3}} u^{2}
$$

by weak convergence. This applies mutatis mutandis to the von Weizsäcker term. On the other hand, for nonquadratic terms such as the $\rho^{5 / 3}$ term, weak convergence is not sufficient. The key point is to remark that, for $\rho \geq 1$,

$$
\text { if } \rho_{n} \text { is bounded in } L^{p}\left(\mathbb{R}^{3}\right) \text { and } \rho_{n} \stackrel{n \rightarrow+\infty}{\longrightarrow} \rho \text { a.e., }
$$

$$
\text { then }\left|\rho_{n}\right|^{p}-\left|\rho_{n}-\rho\right|^{p} \stackrel{n \rightarrow+\infty}{\longrightarrow}|\rho|^{p} \text { in } L^{1}\left(\mathbb{R}^{3}\right) \text {. }
$$


When $p=1$ (4.9) is a straightforward consequence of the Lebesgue dominated convergence theorem. When $p>1$, this property is a corollary of the following one:

$$
\begin{aligned}
& \text { If }\left|f_{n}\right| \leq g_{n} \text { where } g_{n} \text { is weakly compact in } L^{1}\left(\mathbb{R}^{3}\right), \\
& \text { i.e. } \sup _{n}\left(\int_{A} g_{n}+\int_{|x| \geq R} g_{n}\right) \longrightarrow 0 \text { when }|A| \longrightarrow 0, R \longrightarrow+\infty, \\
& \text { and } f_{n} \stackrel{n \rightarrow+\infty}{\longrightarrow} f \text { a.e., then } f_{n} \stackrel{n \rightarrow+\infty}{\longrightarrow} f \text { in } L^{1}\left(\mathbb{R}^{3}\right) .
\end{aligned}
$$

Indeed, in order to establish (4.9), it suffices to use

$$
\left.|| \rho_{n}\right|^{p}-\left|\rho_{n}-\rho\right|^{p}|\leq C| \rho \mid\left(\left|\rho_{n}\right|^{p-1}+|\rho|^{p-1}\right),
$$

for some universal constant $C$, and to apply property (4.10). In turn, (4.10) comes from a direct application of Egorov's Theorem.

Once (4.8) is proven, it remains to remark that

$$
I_{\lambda}\left(\Delta_{n}\right) \geq I_{\mu}\left(z_{1}\right)+I_{\lambda_{n}}\left(z_{2}\right)+\epsilon_{n}
$$

where $\lambda_{n}=\int_{\mathbb{R}^{3}}\left(u_{n}-u\right)^{2}$, and $\epsilon_{n}$ is some error term, and to deduce, letting $n$ go to infinity,

$$
\liminf I_{\lambda}\left(\Delta_{n}\right) \geq I_{\mu}\left(z_{1}\right)+I_{\lambda-\mu}\left(z_{2}\right) .
$$

This concludes the proof of (4.7), and thus that of Assertion (1) of Theorem4.2.

The purpose of Assertions (2) and (3) is to show that one can significantly reduce the number of cases to examine in order to prove (4.2), or in other words that all the values $\mu$ need not be considered.

Let us consider $\mu$ where the infimum

$$
\inf _{\mu \in[0, \lambda]}\left(I_{\mu}\left(z_{1}\right)+I_{\lambda-\mu}\left(z_{2}\right)\right)
$$

is attained. Without loss of generality, we may assume that both minimization problems $I_{\mu}\left(z_{1}\right)$ and $I_{\lambda-\mu}\left(z_{2}\right)$ are indeed attained, respectively for some $\rho_{1}$ and $\rho_{2}$. When $z_{1} \geq \mu$ and $z_{2} \geq \lambda-\mu$, this is clear by an application of Theorem 3.11, but careful arguments actually show that we may always assume this is the case. Let us define $u_{1}=\sqrt{\rho}_{1}$ and $u_{2}=\sqrt{\rho}_{2}$. We claim that necessarily the Lagrange multipliers $\theta_{1}$ and $\theta_{2}$ associated to these minimizers $u_{1}$ and $u_{2}$ are equal to one another, and we henceforth denote by $\theta$ the common value. If $\mu \in] 0, \lambda[$, it is a simple consequence of the observation along which the Lagrange multiplier can be recast as the derivative of the infimum with respect to the constraint: $\theta_{1}=-\frac{d}{d \mu_{1}} I_{\mu_{1}}\left(z_{1}\right)$, and analogously for $\theta_{2}$. The optimality of $\mu$ then reads

$$
\begin{aligned}
0 & =\frac{d}{d \mu}\left(I_{\mu}\left(z_{1}\right)+I_{\lambda-\mu}\left(z_{2}\right)\right) \\
& =\frac{d}{d \mu} I_{\mu}\left(z_{1}\right)-\frac{d}{d \mu} I_{\lambda-\mu}\left(z_{2}\right) \\
& =-\theta_{1}+\theta_{2} .
\end{aligned}
$$

In the case when the optimal $\mu$ is either 0 or $\lambda$, the above simple argument does not apply, but it may still be proven that $\theta_{1}=\theta_{2}$. 
Assume now, as is the case for Assertion (2), that $z_{1}+z_{2}=Z \geq \lambda$. It follows that only three cases may occur:

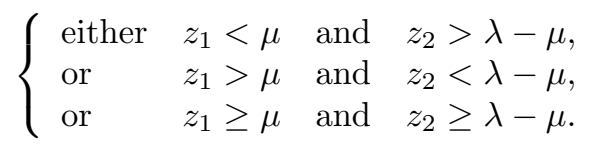

In order to prove Assertion (2), which in the present diatomic case reads

$$
\begin{aligned}
& I\left(\lambda ; z_{1}, z_{2}\right)<\quad \inf \left(I_{\mu}\left(z_{1}\right)+I_{\lambda-\mu}\left(z_{2}\right)\right), \\
& z_{1} \geq \mu \\
& z_{2} \geq \lambda-\mu \\
& \text { same Lagrange multiplier }
\end{aligned}
$$

we have to rule out the first two cases of (4.11), which we now do by proving that in either of the two cases, the strict subadditivity condition automatically holds.

In all of the cases, we have $z_{1} \geq \mu$ or $z_{2} \geq \lambda-\mu$, and thus, by a property of the TFW model, we know that the Lagrange multiplier $\theta_{1}$ or $\theta_{2}$ is positive. As the two of them are identical (and equal to $\theta$ ), this shows that $\theta$ is positive. From this we deduce the exponential fall-off at infinity of both $u_{1}$ and $u_{2}$. This property is a consequence of the following lemma. For the sake of generality, we state it in the molecular case, although we only need it here in the atomic case (replace $-\sum_{k=1}^{K} \frac{z_{k}}{\left|x-\bar{x}_{k}\right|}$ by $-\frac{z_{k}}{|x|}$ below).

Lemma 4.8. Let $\psi>0$ be a solution to

$$
-\Delta \psi-\sum_{k=1}^{K} \frac{z_{k}}{\left|x-\bar{x}_{k}\right|} \psi+\left(\psi^{2} \star \frac{1}{|x|}\right) \psi+c_{1} \psi^{7 / 3}-c_{2} \psi^{5 / 3}+\theta \psi=0,
$$

with $z_{k} \geq 0, \int_{\mathbf{R}^{3}} \psi^{2}=Z$. Let $m=\sqrt{\theta}$. Then there exists some function a, continuous on the unit sphere, such that

$$
\psi(x) \exp (m|x|)|x|-a\left(\frac{x}{|x|}\right) \stackrel{|x| \rightarrow+\infty}{\longrightarrow} 0 .
$$

The proof of this technical lemma falls into two steps. First, a rough estimation is derived that yields the behaviour at infinity of $\psi$ (it is bounded from above by an exponentially decreasing function) and that allows us to show that the potential in (4.13) satisfies

$$
\left|\sum_{k=1}^{K} \frac{z_{k}}{\left|x-\bar{x}_{k}\right|}+\left(\psi^{2} \star \frac{1}{|x|}\right)+c_{1} \psi^{5 / 3}-c_{2} \psi^{4 / 3}\right| \leq \frac{c}{|x|^{2}} .
$$

Second, a technique of supersolution for the elliptic operator

$$
-\Delta+m^{2} \pm \frac{c}{|x|^{2}}
$$

leads to the conclusion.

We now introduce the sequence of functions

$$
u_{\Delta}=\sqrt{\lambda} \frac{u_{1}+u_{2}(\cdot-\Delta \mathbf{e})}{\left\|u_{1}+u_{2}(\cdot-\Delta \mathbf{e})\right\|_{L^{2}}},
$$


which is to be seen as a prototypical example of a loss of compactness. We remark that the energy of this function can be decomposed into

$$
E\left(u_{\Delta}\right)+\frac{z_{1} z_{2}}{\Delta}=I_{\mu}\left(z_{1}\right)+I_{\lambda-\mu}\left(z_{2}\right)+\frac{\left(z_{1}-\mu\right)\left(z_{2}-(\lambda-\mu)\right)}{\Delta}+o\left(\frac{1}{\Delta}\right),
$$

the latter term being a consequence of the exponential behaviour stated in Lemma 4.8

We can then conclude that Assertion (2) holds because, in the first two cases of (4.11), the term $\left(z_{1}-\mu\right)\left(z_{2}-(\lambda-\mu)\right) / \Delta$ in (4.16) is negative and thus for $\Delta$ large enough we obtain the strict subaddivity condition.

Assertion (3) is an easy consequence of Assertion (2) since the case $\left(z_{1} \geq \mu\right.$, $\left.z_{2} \geq \lambda-\mu\right)$ is restricted to $\left(z_{1}=\mu, z_{2}=\lambda-\mu\right)$ when $z_{1}+z_{2}=\lambda$.

We now address Assertion (4). It is to be noted that all of the first three assertions indeed hold for the TF model (through slight modifications of the above arguments), but that the fourth one is not true for the TF model because there is then a special role played by the von Weizsäcker term.

We want to prove that, under the assumption $Z=\lambda$, there exists some $\Delta$ such that

$$
I_{\lambda}(\Delta)+\frac{z_{1} z_{2}}{\Delta}<I_{z_{1}}\left(z_{1}\right)+I_{z_{2}}\left(z_{2}\right)
$$

where it is understood that both minimization problems in the r.h.s. share the same Lagrange mutiplier.

This is a rather involved calculation to prove that, for some $\Delta$ large enough,

$$
\begin{aligned}
& E\left(u_{\Delta}\right)+\frac{z_{1} z_{2}}{\Delta} \\
& =I_{z_{1}}\left(z_{1}\right)+I_{z_{2}}\left(z_{2}\right) \\
& -\left(\lim _{\Delta \longrightarrow+\infty} e^{m \Delta} \Delta \int_{\mathbb{R}^{3}} \nabla u_{1}(\cdot) \nabla u_{2}(\cdot-\Delta \mathbf{e})+m^{2} \int_{\mathbb{R}^{3}} u_{1}(\cdot) u_{2}(\cdot-\Delta \mathbf{e})\right) \frac{e^{-m \Delta}}{\Delta} \\
& +o\left(\frac{e^{-m \Delta}}{\Delta}\right) \text {. }
\end{aligned}
$$

The evaluation of the error term again comes from the exponential decay of $u_{1}$ and $u_{2}$, but now some more precise information is needed on the behaviour at infinity of the functions $u_{i}$ in order to prove that the term

$$
\lim _{\Delta \longrightarrow+\infty} e^{m \Delta} \Delta \int_{\mathbb{R}^{3}} \nabla u_{1}(\cdot) \nabla u_{2}(\cdot-\Delta \mathbf{e})+m^{2} \int_{\mathbf{R}^{3}} u_{1}(\cdot) u_{2}(\cdot-\Delta \mathbf{e})
$$

is indeed positive, which will conclude the proof of Assertion (4), and thus that of the theorem.

A number of technical results, in the spirit of the above Lemma 4.8, allows us to evaluate the asymptotic behaviour, as $\Delta$ goes to infinity, of integrals of the type

$$
n t_{\mathbf{R}^{3}} u_{1}(\cdot) u_{2}(\cdot+\Delta \mathbf{e})
$$

which basically are convolution terms. This in turn leads to (4.18).

We would like to comment on (4.18) in two respects. First, it shows that the binding force is exponentially decreasing with respect to the distance (this is exactly the third term of the right-hand side of (4.18), which is not a physical property, as it should decrease only in a polynomial way. Second, we need to remark that this binding force not only has the wrong scaling law but in addition does not originate from the electrostatic term $\left(z_{1}-\mu\right)\left(z_{2}-(\lambda-\mu)\right) / \Delta$, which vanishes in 
the neutral case. The binding force comes from the other terms. Moreover, it does not come from the superquadratic terms $u^{10 / 3}-u^{8 / 3}$ but from the only quadratic term, namely that corresponding to the operator $-\Delta+m^{2}$. These two observations show some dramatically nonphysical behaviour of the TFW model: it allows for binding of molecules, but for some bad reasons. Although it is not proven in its entire generality (see a heuristic argument in 7]), the above two observations are likely to extend to all models of DFT type. We shall see below that the situation is quite different in the Hartree case.

\section{Remark 4.9. On the stability for the $N$-body problem}

It must be mentioned that for the case of the $N$-body problem, E.H. Lieb and W. Thirring showed the stability, i.e. the existence of a minimizing configuration, by showing that there exists at least one configuration that yields an energy strictly less than the sum of the energies of the two systems at infinity, i.e. the analogous statement to (4.6)-(4.7). In this setting, they also established (see [163]) that, up to a rotation of one system with respect to the other, the binding force is at least a polynomial function with respect to the distance separating the two systems, which corroborates the Van der Waals interaction law. In this respect, the $N$-body model is thus qualitatively better than the TFW theory where the binding force scales exponentially with respect to the distance, a highly nonphysical property.

4.3. Generalization to $M>2$ nuclei. We now would like to briefly indicate how the above arguments have to be modified in order to deal with the case of $M>2$ nuclei. A natural idea (although it is not the only one, and we could alternatively adopt a direct strategy based upon the concentration compactness principle - we will come back to that below) is to argue by induction, to suppose that, up to $M-1$ nuclei, all assertions of Theorem 4.2 hold true, and to consider the case of $M$ nuclei. The point is of course Assertion (4), on which we focus.

The main difference lies in the fact that a nontrivial product of a possible dissociation is not an atom, but a molecule, and therefore intricate geometrical arguments come into play. Indeed, suppose the system splits into $\left(\lambda_{1}, z_{i_{1}}, \ldots, z_{i_{p}}\right)$, $\left(\lambda_{2}, z_{i_{p+1}}, \ldots, z_{i_{M}}\right)$, with $\lambda_{1}+\lambda_{2}=\lambda,\left\{i_{1}, \ldots, i_{M}\right\}=\{1, \ldots, M\}$. Suppose also, to fix the ideas, that each of the subsystems gives rise to a well-posed minimization problem for the electrons, in the sense that there are a minimizing $\rho_{1}$ and a minimizing $\rho_{2}$ (and we have already mentioned that we may always restrict ourselves to this case). If we now wish to apply the same argument, we will consider a recombination, formerly denoted by $u_{\Delta}$ in (4.15), of the two minimizers $\rho_{1}$ and $\rho_{2}$. The difficulty here originates from the fact that, contrary to the diatomic case, there are many ways to cluster two molecules: the way one gets closer to the other one has an impact on the electrostatic balance, and thus on the overall energy of the recombined system. This can be very well understood intuitively, as chemistry most often proceeds from geometrical considerations such as aligning and orienting molecules in a convenient way so that they react together. As it is impossible to guess which way is the best to recombine the system, we then argue by taking the spherical average over all possible geometrical arrangements. More precisely, for two arbitrary rotations $R$ and $S$, we consider the subsystems $\left(\rho_{1}\left(R^{-1} \cdot\right) ; R \bar{x}_{i_{1}}, \ldots, R \bar{x}_{i_{p}}\right)$ and $\left(\rho_{1}\left(S^{-1}\right) ; S \bar{x}_{i_{p+1}}, \ldots, S \bar{x}_{i_{M}}\right)$, and next recombine them at the distance $\Delta$ by 
considering the system

$$
\begin{gathered}
\left(\rho_{\Delta}=\lambda \frac{\left(\sqrt{\rho}_{1}\left(R^{-1} \cdot\right)+\sqrt{\rho}_{2}\left(S^{-1}(\cdot+\Delta \mathbf{e})\right)\right)^{2}}{\left\|\sqrt{\rho}{ }_{1}\left(R^{-1} \cdot\right)+\sqrt{\rho_{2}}\left(S^{-1}(\cdot+\Delta \mathbf{e})\right)\right\|_{L^{2}}^{2}}\right. \\
\left.R \bar{x}_{i_{1}}, \ldots, R \bar{x}_{i_{p}}, \Delta \mathbf{e}+S \bar{x}_{i_{p+1}}, \ldots, \Delta \mathbf{e}+S \bar{x}_{i_{M}}\right) .
\end{gathered}
$$

In order to check that there exist some convenient rotations $R$ and $S$ such that, for $\Delta$ large enough, the energy balance

$$
I_{\lambda}(\mathcal{S})<I_{\mu}\left(\mathcal{S}_{1}\right)+I_{\lambda-\mu}\left(\mathcal{S}_{2}\right)+I(R, S, \Delta)
$$

(with a self-explanatory notation) is favorable to recombination (so that the strict subadditivity condition is proven), it suffices to show that the average over all possible $R$ and $S$ is negative, i.e.

$$
\iint I(R, S, \Delta) d \mu(R) d \mu(S)<0
$$

(thus at least for some $R$ and $S$ and in fact for a set of positive measure of them, the energy balance will be negative). This is in turn obtained by averaging the estimations done on the $M=2$ case.

4.4. Adaptation to the $\mathbf{H}$ and $\mathbf{H F}$ cases. Regarding the Hartree and HartreeFock cases, the proof of Theorem 4.6 basically follows the same lines as that of Theorem 4.2 with many more technicalities though (in particular due to the fact that the wavefunctions in the $\mathrm{H}$ and $\mathrm{HF}$ model are not signed functions, contrary to the minimizing $u=\sqrt{\rho}$ of TF-like models). The main two differences are, on the one hand, that only dissociations into two subsystems with an integer number of electrons have to be considered, and, on the other hand, the proof of Assertion (4) collapses in the Hartree-Fock case. In fact, as already mentioned above, the fact that integer dissociations are the only ones to prevent is due to the concavity of the Hartree and Hartree-Fock models with respect to the mass constraint. A worst case analysis leads to the conclusion that integer dissociations are those to prevent, and the proof thus concentrates on them.

In the Hartree case, Assertion (4) can be proven. Beyond the details of the proof, one interesting feature is that in this model, and contrary to the ThomasFermi type models, the binding force is of electrostatic nature. It comes from the electron interaction term and not from the other terms, as e.g. in the TFW case seen above. However, no precise estimate on the binding force has been given to date.

Let us only outline the proof of Assertion (4). As above in (4.17), we need to prove that there exists a way to combine the two subsystems in a manner such that the energy decreases (w.r.t. the situation where the two are taken infinitely far away from one another). For this purpose, some convenient geometrical arrangement must be found, as in 4.19), i.e. a convenient distance $\Delta$ and also a convenient orientation, expressed by the two rotations $R$ and $S$. The technical novelty with respect to the recombination (4.19) is that the ground state of each subsystem is not defined solely by the densities $\rho_{i}, i=1,2$, but by two $N$-tuples $\left(\phi_{1}^{i}, \ldots, \phi_{N}^{i}\right.$ ) (with $\left.\sum_{j=1}^{N}\left|\phi_{j}^{i}\right|^{2}=\rho_{i}\right)$. The key difference with the TF-type theory is that the energy balance term $I(R, S, \Delta)$ is here due to the electrostatic balance, as announced above. 
Indeed, it can be seen that

$$
I(R, S, \Delta)=\left(\frac{1}{|x|} \star\left(\rho_{1}-m_{1}\right) \star\left(\hat{\rho}_{2}-\hat{m}_{2}\right)\right)(\Delta),
$$

where $m_{i}$ is the measure defining the nuclei of subsystem $i$, i.e.

$$
m_{i}=\sum_{j \in S_{i}} \delta\left(\cdot-\bar{x}_{j}\right),
$$

and $\hat{f}(x)$ denotes the function $f(-x)$. The proof of Assertion (4) amounts to proving that $I(R, S, \Delta)$ is negative for some appropriate choice of its arguments. This is performed by first taking the spherical average

$$
\begin{aligned}
I(\Delta) & =\overline{I(R, S, \Delta)}=\iint I(R, S, \Delta) d \mu(R) d \mu(S) \\
& =\left(\frac{1}{|x|} \star\left(\bar{\rho}_{1}-\bar{m}_{1}\right) \star\left(\bar{\rho}_{2}-\bar{m}_{2}\right)\right)(\Delta)
\end{aligned}
$$

of $I(R, S, \Delta)$ (where $\bar{f}$ denotes the spherical average of $f$ ) as in 4.21), and then showing that $I(\Delta)$ is negative for some $\Delta$. The latter is proven by showing first that

$$
\int I(\Delta) d \Delta=0
$$

This fact is a consequence of the very definition (4.22) along with $\int \rho_{i}=m_{i}\left(\mathbb{R}^{3}\right)$, $i=1,2$, and the property that $\bar{\rho}_{i}$ decays exponentially fast at infinity, which in turn comes from the fact that the $\phi_{j}^{i}, j=1, \ldots, N$ satisfy the Euler-Lagrange equation with a positive Lagrange multiplier since they form the ground state of subsystem $S_{i}$. On the other hand $I(\Delta)$ cannot identically vanish; otherwise, again by (4.22) and due to the smoothness of $\rho_{i}$, we would have e.g. $\rho_{1}=m_{1}$. This concludes the proof.

In the case when the two subsystems are planar (not necessarily coplanar) an alternative proof can be provided that shows that, for $\Delta$ large enough, $I(\Delta)<0$, the argument requiring a sharp asymptotic expansion as $|x| \longrightarrow+\infty$ of $\phi_{i}$, in the spirit of Lemma 4.8 .

On the other hand, an analogous recombination of two subsystems in the HartreeFock setting presents an unsolved mathematical problem: it is not known how to deal with the orthonormality constraint, and the proof of Assertion (4) therefore remains an unsolved mathematical question.

4.5. On the concentration-compactness method. A useful rereading of the previous proofs consists of the following. We have brought down the stability of a system (or in mathematical words the compactness of the associated minimization problem) into two steps:

- the identification of a necessary and sufficient condition consisting of the strict subadditivity condition (4.2),

- the estimation of interaction laws in order to prove that the strict subadditivity condition holds, under convenient conditions.

This scheme of proof applies to many contexts and is the essence of the concentration-compactness method, first introduced in [167, 168]. 
As a further example of the power of this general strategy, we briefly come back to a setting hardly approached above, that of the electronic problem for the TFDW problem (note that we come back to a setting where nuclei are clamped at fixed positions). Another instance of applications of concentration-compactness is nuclear physics: in [120], the possible fission of a nucleus is investigated with this method.

For convenience, let us recall the TFDW problem already introduced in (3.47):

with

$$
I_{\lambda}^{T F D W}=\inf \left\{E_{T F D W}(\rho), \quad \rho \geq 0, \quad \sqrt{\rho} \in H^{1}\left(\mathbb{R}^{3}\right), \quad \int_{\mathbf{R}^{3}} \rho=\lambda\right\},
$$

$$
\begin{aligned}
E_{T F D W}(\rho)= & C_{W} \int_{\mathbb{R}^{3}}|\nabla \sqrt{\rho}|^{2}+C_{T F} \int_{\mathbb{R}^{3}} \rho^{5 / 3}+\int_{\mathbf{R}^{3}} \rho V \\
& +\frac{1}{2} \int_{\mathbb{R}^{3}} \int_{\mathbb{R}^{3}} \frac{\rho(x) \rho(y)}{|x-y|} d x d y-C_{D} \int_{\mathbb{R}^{3}} \rho^{4 / 3} .
\end{aligned}
$$

It is easy to see that, due to the $-C_{D} \int_{\mathbf{R}^{3}} \rho^{4 / 3}$ term, the functional is not weakly lower semicontinuous for the $H^{1}$ weak topology. Consequently, for the weak limit $\rho$ of a minimizing sequence, we do not have any longer the very useful information $E^{T F D W}(\rho) \leq I_{N}^{T F D W}$. In addition, again because of the Dirac term, we cannot relax the mass constraint so easily, and prove by pushing some mass to infinity that we may replace the equality constraint $\int_{\mathbb{R}^{3}} \rho=\lambda$ by an inequality $\int_{\mathbb{R}^{3}} \rho \leq \lambda$. It follows that we lack information on the tentative minimizer $\rho$.

A more precise argument is in order. Here, we must indeed estimate (as we did above for the geometry optimization problem) which part of the energy is carried by some piece of the minimizing sequence $\rho_{n}$ possibly escaping at infinity. For this purpose, we introduce the problem at infinity

$$
I_{\lambda}^{T F D W, \infty}=\inf \left\{E_{T F D W, \infty}(\rho), \quad \rho \geq 0, \quad \sqrt{\rho} \in H^{1}\left(\mathbb{R}^{3}\right), \quad \int_{\mathbb{R}^{3}} \rho=\lambda\right\},
$$

where

$E_{T F D W, \infty}(\rho)=$ identical to $E_{T F D W}(\rho)$ but with the potential $V$ set to zero.

This translates the fact that the electronic density $\rho$, escaped at infinity, does not interact with the nuclei.

Then, in the spirit of what has been done for the geometry optimization problem (note that the proof of the existence of a minimizer for the TFDW problem, published in [166] is nevertheless anterior to that of the existence of the optimal geometry), it can be shown that a necessary and sufficient condition for all minimizing sequences of the TFDW problem to be relatively compact reads

$$
I_{\lambda}^{T F D W}<I_{\mu}^{T F D W}+I_{\lambda-\mu}^{T F D W, \infty}
$$

for all $\mu \in[0, \lambda[$. This condition is rather intuitive, as it compares the energy of the system under consideration with the sum of the energies of two subsystems, one at finite distance ( $\mu$ electrons have stayed in the vicinity of the nuclei, this corresponds to the weak limit of the minimizing sequence), and the other one at infinity (the loss of mass in the constraint $\int_{\mathbb{R}^{3}} \rho \leq \lambda$ corresponds to $\lambda-\mu$ electrons). 
This condition is in turn established for $Z \geq \lambda$, again by carefully examining the interaction potential between two subsystems at large distance. It can indeed be proven that, for spectral reasons, the minimizing sequence may only separate into a finite number of pieces and that each of the subsystems is compact (i.e. each of the minimization problems created by the splitting is attained). Again, a crucial role is played by the positivity of the Lagrange multipliers, which enforces the exponential decay of the minimizers for the subsystems, and thus allows for the precise calculations of the interaction.

Note that one of the difficulties mentioned above was that no information could be obtained at once on the weak limit of minimizing sequences (contrary to the case say of the TFW model, where it is shown that the weak limit is a minimizer for the problem with relaxed constraint). In fact, such information is indeed present in the problem, when one looks at the good minimization problem, which is here $I_{\mu}^{T F D W}$ and not $I_{\lambda}^{T F D W}$ with relaxed constraint, again because of the nonconvexity of the functional.

\section{Remark 4.10. On the Kohn-Sham model}

We would like to recall that the same approach allows us to prove the existence of a ground state for the Kohn-Sham model (see [140]).

\section{A Detour By TIME-DEPEnDEnT MODELS}

Analogous comments to those made in the static setting hold on the difficulty of directly approximating numerically the time-dependent version of (2.1):

$$
i \frac{\partial \psi}{\partial t}=H \psi
$$

A commonly used approach to circumvent the dimensionality problem is to resort to the ab initio molecular dynamics equations. In this approximation, one solves the time evolution of the molecular structure according to the Newton law of classical mechanics

$$
m_{k} \frac{d^{2} \bar{x}_{k}}{d t^{2}}(t)=-\nabla_{\bar{x}_{k}} W\left(\bar{x}_{1}(t), \cdots, \bar{x}_{M}(t)\right)
$$

where the potential $W$ comes both from the potential created by the electrons of the system and from the internuclear repulsion:

$$
W\left(\bar{x}_{1}, \cdots, \bar{x}_{M}\right)=U\left(\bar{x}_{1}, \cdots, \bar{x}_{M}, t\right)+\sum_{1 \leq k<l \leq M} \frac{z_{k} z_{l}}{\left|\bar{x}_{k}-\bar{x}_{l}\right|} .
$$

There remains then to know how to calculate the potential $U\left(\bar{x}_{1}, \cdots, \bar{x}_{M}, t\right)$, and this is where different models exist. We will briefly overview here some of the mathematical works dealing with the various models employed at this level. Doing so, we concentrate on the quantum level, i.e. the determination of the potential $U\left(\bar{x}_{1}, \cdots, \bar{x}_{M}, t\right)$ in a quantum setting, and its coupling with the classical description of the evolution of the nuclei. On the other hand, the mathematical study of the case when $U\left(\bar{x}_{1}, \cdots, \bar{x}_{M}, t\right)$ is modelled at a simple classical level, and that of the classical evolution of the nuclei are left aside. The latter problems are much easier mathematically, for they involve ODEs rather than PDEs. Notice however that they give birth to the enormous field of molecular dynamics, and thus to an incredible number of numerical issues. 
In its entire generality, the potential $U$ comes from the time evolution of the electronic wavefunction, dictated by the time-dependent Schrödinger equations for the electronic wavefunction:

$$
i \frac{\partial \psi_{e}}{\partial t}=H_{e}^{\left(\bar{x}_{1}(t), \cdots, \bar{x}_{M}(t)\right)} \psi_{e}
$$

The coupled system to simulate thus reads

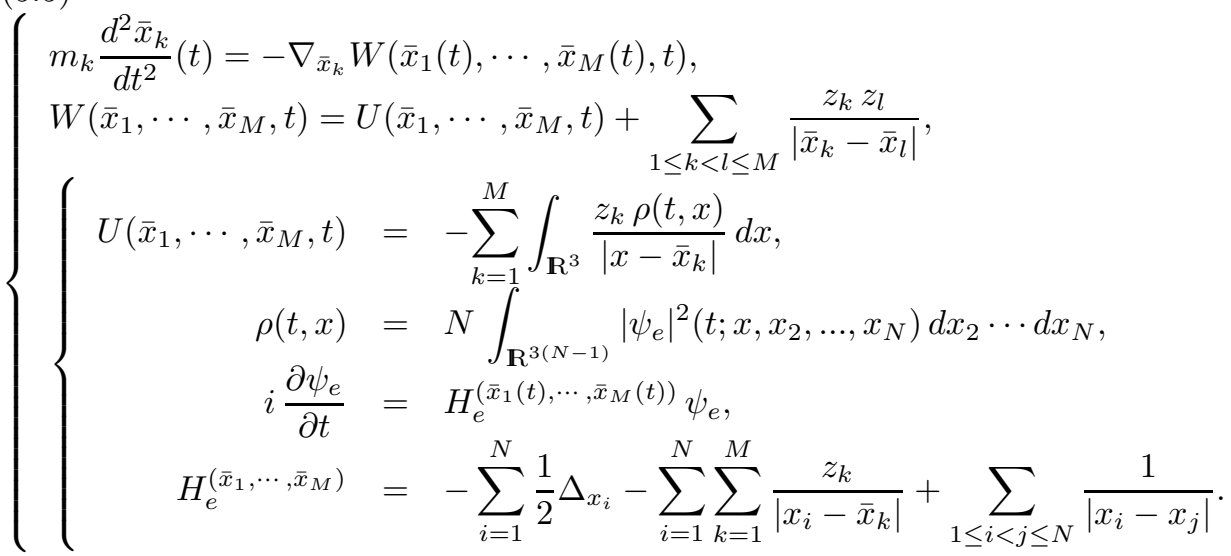

We refer to [36] for a mathematical analysis of the relation between (5.5) and the original problem (5.1). This approximation is called the nonadiabatic approximation, as opposed to the adiabatic approximation, which we now see.

The adiabatic approximation consists of assuming that for any time $t$ the electrons remain in the ground state (or in some well-defined excited state) of the electronic Hamiltonian $H_{e}^{\left(\bar{x}_{1}(t), \cdots, \bar{x}_{M}(t)\right)}$. The electrons are said to evolve on a given Born-Oppenheimer energy surface. Even if electronic nonadiabatic effects are important in some fundamental molecular processes such as collisions, the adiabatic approximation is valid in many cases. In this approximation, the last four lines of (5.5) are replaced by

$$
\left\{\begin{aligned}
U\left(\bar{x}_{1}, \cdots, \bar{x}_{M}\right) & =\inf \left\{\left\langle\psi_{e}, H_{e}^{\left(\bar{x}_{1}, \cdots, \bar{x}_{M}\right)} \psi_{e}\right\rangle, \quad \psi_{e} \in \mathcal{H}_{e}, \quad\left\|\psi_{e}\right\|_{L^{2}}=1\right\}, \\
H_{e}^{\left(\bar{x}_{1}, \cdots, \bar{x}_{M}\right)} & =-\sum_{i=1}^{N} \frac{1}{2} \Delta_{x_{i}}-\sum_{i=1}^{N} \sum_{k=1}^{M} \frac{z_{k}}{\left|x_{i}-\bar{x}_{k}\right|}+\sum_{1 \leq i<j \leq N} \frac{1}{\left|x_{i}-x_{j}\right|} .
\end{aligned}\right.
$$

It is not easy to study the mathematical properties of this approximation and its connection with the nonadiabatic setting. Particularly intricate is the analysis of the so-called energy crossings, i.e. the pathological cases when the Born-Oppenheimer energy surfaces happen to cross each others for some given configuration of nuclei. For a mathematical study of this approximation, we refer to [124, 125, 38].

In practice, again because of the high dimension of the electronic problem, both systems (5.5) and (5.6) have to be further approximated.

As in the previous sections for the time-independent case, the minimization problem (5.6) is approximated by one of the standard methods, HF or DFT. The 
system obtained then reads

$$
\begin{cases}m_{k} \frac{d^{2} \bar{x}_{k}}{d t^{2}}(t)= & -\nabla_{\bar{x}_{k}} W\left(\bar{x}_{1}(t), \cdots, \bar{x}_{M}(t)\right), \\ W\left(\bar{x}_{1}, \cdots, \bar{x}_{M}\right)= & U\left(\bar{x}_{1}, \cdots, \bar{x}_{M}\right)+\sum_{1 \leq k<l \leq M} \frac{z_{k} z_{l}}{\left|\bar{x}_{k}-\bar{x}_{l}\right|}, \\ U\left(\bar{x}_{1}, \cdots, \bar{x}_{M}\right) & \text { evaluated in a given static model. }\end{cases}
$$

With a view to circumvent the difficulty of solving a minimization problem (i.e. in practice a nonlinear eigenvalue problem) at each time step, which turns out to be more difficult than advancing forward in time an evolution electronic Schrödinger equation, the idea has arisen to replace the minimization by a virtual time evolution. This is the Car-Parrinello dynamics, introduced in [58]. It consists of replacing the minimization problem by a fictitious (nonphysical) electronic dynamics which makes the electronic wave function evolve in the neighbourhood of the adiabatic state. Heuristically, the method is close to the notion of relaxing a holonomous constraint in classical dynamics. From a mathematical point of view, the CarParrinello method is investigated in [37. Explicitly, the last four lines of (5.5) are replaced by

$$
\left\{\begin{aligned}
U\left(\bar{x}_{1}, \cdots, \bar{x}_{M}, t\right) & =E_{\bar{x}_{1}, \cdots, \bar{x}_{M}}^{K S}\left(\phi_{1}(t), \ldots, \phi_{N}(t)\right) \\
\mu \frac{\partial^{2} \phi_{i}}{\partial t^{2}}(t) & =-K\left(\rho_{\Phi(t)}\right) \phi_{i}(t)+\sum_{j=1}^{N} \Lambda_{i j}(t) \phi_{j}(t) \\
\Lambda_{i j}(t) & =\left\langle\phi_{j}(t), K\left(\rho_{\Phi(t)}\right) \phi_{j}(t)\right\rangle-\mu \int_{\mathbb{R}^{3}} \frac{\partial \phi_{j}}{\partial t}(t) \frac{\partial \phi_{i}(t)}{\partial t}(t),
\end{aligned}\right.
$$

where $\mu$ is a fictitious mass that needs to be properly fitted to reach efficiency in the computations. In particular, it is chosen much larger than the electron mass $\left(m_{e}=1\right)$. This allows one to numerically integrate (5.8) with a larger timestep than that for the nonadiabatic simulations, thus giving a more efficient method. On the other hand, although the timestep used for (5.8) needs to be smaller than that used for the adiabatic simulation, the Car-Parrinello method is also more efficient than the adiabatic ones because no minimization is required. Formally, the limit $\mu \longrightarrow 0$ allows one to recover the adiabatic approximation, as may be understood by looking at the last two lines of (5.8).

Less clear is the way to further approximate the nonadiabatic system (5.5). This requires us to concentrate on the approximation of the time-dependent Schrödinger equation for electrons (5.4).

A commonly used approximation is the time-dependent Hartree-Fock model. It consists of the system of evolution equations

$$
\left\{\begin{aligned}
i \frac{\partial \phi_{i}}{\partial t}= & -\frac{1}{2} \Delta \phi_{i}-\sum_{k=1}^{M} \frac{z_{k}}{\left|\cdot-\bar{x}_{k}(t)\right|} \phi_{i}+\left(\sum_{j=1}^{N}\left|\phi_{j}\right|^{2} \star \frac{1}{|x|}\right) \phi_{i} \\
& -\sum_{j=1}^{N}\left(\phi_{j}^{*} \phi_{i} \star \frac{1}{|x|}\right) \phi_{j}
\end{aligned}\right.
$$

supplied with some initial condition $\phi_{i}(0)=\phi_{i}^{0}$. These equations are obtained as the stationary condition for the action when the wave function $\psi_{e}$ solution to (5.4) is bound to evolve on the manifold of Slater determinants. The well-posedness of the Cauchy problem (for fixed nuclei $\bar{x}_{k}$ ) has been proven by Chadam and Glassey in 
70]. The relation between the time-dependent Hartree-Fock equation and the original Schrödinger equation is mostly unclear (mathematically). In fact, the TDHF equation is the simplest way to close the time-dependent Schrödinger hierarchy. This hierarchy is obtained by successively integrating the Schrödinger equation, multiplied by $\psi_{e}$, over any subset of $p$ electrons amongst the $N$ electrons (recall we have seen the static version of this technique in Remark 3.13). An evolution equation on each of the density matrices (marginal distributions) at all orders is therefore obtained. The Hartree-Fock approximation amounts to approximating the matrix of order 2 by an antisymmetrized product built with the matrix of order 1. It therefore closes the hierarchy at the lowest possible order. By studying the TDHF equation as a closure of the Schrödinger hierarchy, C. Bardos, F. Golse and collaborators (see [9] and other references by the same authors) have recently shown that the TDHF equation approaches the Schrödinger equation in the limit of an infinite number of electrons. However, the proof applies only for well-prepared initial states (Slater determinants, and slightly more general initial data), and only in the weak coupling picture. The latter assumption means that a factor $1 / N$ is inserted in front of the electronic interaction term $\sum_{i \neq j} \frac{1}{\left|x_{i}-x_{j}\right|}$ in the Hamiltonian $H_{e}$ so that each electron only experiences a force (a potential) of size $O(1)$ and not of order $N$. Note that, as often, the same proof in the setting of the Hartree model, is significantly simpler.

In this vein, an interesting approach would be to stay in the strong coupling framework (no factor $1 / N$ ) but to try to prepare data such that the electrons spontaneously stay at a controlled distance and arrange themselves (in a classic picture) so that they experience a force $O(1)$. In particular, this might be possible in the presence of nuclei.

Once the time-dependent Hartree-Fock equation is inserted in a coupled evolution with nuclei, we obtain the system

$$
\left\{\begin{aligned}
m_{k} \frac{d^{2} \bar{x}_{k}}{d t^{2}(t)}= & -\nabla_{\bar{x}_{k}} W\left(\bar{x}_{1}(t), \cdots \bar{x}_{M}(t), t\right), \\
\bar{x}_{k}(0) & =\bar{x}_{k}^{0} \\
\frac{d \bar{x}_{k}}{d t}(0) & =\bar{v}_{k}^{0} \\
W\left(\bar{x}_{1}, \cdots \bar{x}_{M}, t\right) & =-\sum_{k=1}^{M} \sum_{i=1}^{N} z_{k} \int \frac{\left|\phi_{i}(t, x)\right|^{2}}{\left|x-\bar{x}_{k}\right|} d x+\sum_{1 \leq k<l \leq M} \frac{z_{k} z_{l}}{\left|\bar{x}_{k}-\bar{x}_{l}\right|}, \\
i \frac{\partial \phi_{i}}{\partial t} & =-\frac{1}{2} \Delta \phi_{i}-\sum_{k=1}^{M} \frac{z_{k}}{\left|\cdot-\bar{x}_{k}(t)\right|} \phi_{i}+\left(\sum_{j=1}^{N}\left|\phi_{j}\right|^{2} \star \frac{1}{|x|}\right) \phi_{i} \\
& -\sum_{j=1}^{N}\left(\phi_{j}^{*} \phi_{i} \star \frac{1}{|x|}\right) \phi_{j} .
\end{aligned}\right.
$$

It is proven in 52] that the Cauchy problem for (5.10) is well-posed, provided that the initial conditions $\phi_{i}^{0}$ are chosen regular enough. This assumption is not restrictive because in practice the initial data are often eigenstates of an electronic Hamiltonian, thus regular functions.

\section{Remark 5.1. On the control of molecular evolutions}

One may modify any of the above systems with a view to modelling a molecular system subject to an electric field. In the framework of perturbation theory, it 
suffices to insert some term of the type

$$
-E(t, x) \psi_{e}
$$

in the right-hand side of the electronic Schrödinger equation. The approximated systems can be modified correspondingly. A typical case of relevance for such models is the control of molecular evolutions, and beyond, that of chemical reactions. The electric field is then obtained by a laser shining on the molecular system under study. For an introduction to the challenges of this emerging field, we refer to the book [10] and the introductory survey [145] therein. There are a few mathematical works dealing with such situations: we wish to mention [55] for an existence of an optimal control in this setting, together with works by J.-P. Puel and collaborators [11, 12, 13, for an extension. For the exact control (note the problem is bilinear as $E$ multiplies $\psi_{e}$ ), we refer to a series of works by G. Turinici and collaborators (see [202] and references therein).

\section{From molecules to CRYstals}

6.1. The bulk limit problem. Let us come back to the static setting. As a natural follow-up for the geometry optimization problem, stands the following question: can one have some mathematical insight on the reason why matter at zero temperature arranges in periodic crystals? This so-called crystal problem is a cornerstone of physics. Unfortunately, nothing or almost nothing is known at the theoretical level.

Suppose we give ourselves a system of $N$ identical nuclei of unit charge (for simplicity) and provide them with $N$ electrons, suppose they form a stable neutral system in the setting of any model for which the geometry optimization problem has been answered positively in Section 4. Suppose we now let $N$ go to infinity: why are crystal geometries favored energetically in this limit?

The mathematical literature is really poor on the subject, whatever the model chosen. Already for simpler models than the $a b$ initio models treated here, i.e. models based upon two-body interactions, the results are rare: [203, 174, 175], 118] in one dimension, [184 in two dimensions. For the type of models we are interested in for this survey, the only result we are aware of is an oversimplified one-dimensional TF-type model, settled in [25]. It is proven there that any ground state is indeed periodic, and the proof relies on a simple application of the Cauchy uniqueness theorem for ODEs. The technique of proof unfortunately collapses in dimensions 2 and 3 and gives no insight on the result there. Just to mention it, let us indicate that proving periodicity for a TF-like model in 3D basically amounts to studying the Euler-Lagrange equation of the problem, which reads as a system of the form

$$
\left\{\begin{array}{l}
-\Delta u+u^{p}=\sum_{\bar{x}_{k}} \delta\left(\cdot-\bar{x}_{k}\right), \\
u \geq 0, \\
\left.\nabla\left(u-\frac{1}{\left|\cdot-\bar{x}_{k}\right|}\right)\right|_{x=\bar{x}_{k}}=0, \quad \text { for all } k,
\end{array}\right.
$$

and showing that the existence of a solution $\left(u, \bar{x}_{1}, \ldots, \bar{x}_{k}, \ldots\right)$ to this system indeed imposes that the $\bar{x}_{k}$ are periodically arranged. The first two lines indeed translate the optimality of the electronic density, while the third line translates that of the positions of nuclei. The difficulty of the mathematical enterprise is likely to be 
overwhelming. With a view to proceeding further in the program consisting in determining the macroscopic limit of microscopic systems, some ways to circumvent the difficulty will be mentioned in Section $[7$

Of course, numerical experiment could provide an alternative to mathematical arguments. At least it might help to figure out the result. For models based upon two-body interactions (or more) such as particles interacting via a Lennard-Joneslike potential, the literature reports on many numerical experiments. Depending on the potential used for interaction, the ground-state structure might be periodic, or almost periodic, or neither.

In the absence of mathematical evidence, or at least of a convincing piece of understanding, of the periodicity of the set of nuclei, one is left with postulating, e.g. on the basis of experimental observation, that such a periodicity exists, and that it also holds, and here is the delicate point, for the mathematical model under consideration.

In spite of this simplification, the mathematical content of the problem is far from being empty. One indeed needs to establish that the electronic ground state also becomes periodic in the limit, and one needs to properly define the energy of this infinite periodic arrangement of nuclei, together with all the electrons they bind.

The basic question of interest is to define such an energy, and as well to check that there exists a minimizer for it in terms of electronic structure, both in a consistent way with the models for the molecular systems of finite size. Doing so, one aims at a threefold goal:

- first, rigorously define the energy,

- second, show the robustness of the molecular model as the size increases,

- third, assess the validity of the models for the crystalline phase that are used for numerical simulation (see the introductory article [20], and beyond, the treatises [2, 134 for the physical background, 90, 181] for the details of computational strategies).

The mathematical construction we are interested in reads as follows. Consider $\mathbb{Z}^{3}$ as a prototype example of a periodic lattice. Then define a finite subset $\Lambda_{N}$ (this is a historic notation) of $N$ points of the lattice $\mathbb{Z}^{3}$, by intersection of the lattice with a large cube (this is for simplicity, as any sequence of domains, for which the number of sites on the boundary is asymptotically negligible with respect to the number of those in the interior, would be convenient; such domains are called Van Hove sequences in statistical mechanics). Assign a nucleus of unit charge (again this is for simplicity) to each of the points of $\Lambda_{N}$, and consider a well-posed model for the neutral molecular system of finite size composed of the $N$ nuclei plus $N$ electrons. To fix the ideas, suppose it is a Thomas-Fermi-type model, and define

$$
I^{T F, T F W}\left(\Lambda_{N}\right)=I_{N}^{T F, T F W}+\frac{1}{2} \sum_{\bar{x}_{j} \neq \bar{x}_{k} \in \Lambda_{N}} \frac{1}{\left|\bar{x}_{j}-\bar{x}_{k}\right|},
$$

the ground-state energy, i.e. the sum of the electronic energy plus the nuclear term, and let $\rho_{N}$ denote the minimizing density; thus $\int_{\mathbb{R}^{3}} \rho_{N}=N$. For a Hartree-Focktype model, the density matrix $\rho_{N}(x, y)$ would play the main role. 
The so-called thermodynamic limit problem (a more appropriate name is the bulk limit problem, as no finite temperature effect is considered here) consists of asking the following two questions on limits as $N$ grows to infinity:

- (i) does the energy per unit volume $\frac{I\left(\Lambda_{N}\right)}{N}$ converge to a limit? which limit?

- (ii) does the density (or respectively the density matrix) converge to a limit?

To some extent, the question under consideration is both related to fundamental questions of thermodynamics (see 8] for the background) and to the question of defining effective properties of composite materials on the basis of the knowledge of the properties of their constitutive materials.

It is expected that there is some consistency between the limit of the energy and the density in the following sense: in good cases, the energy will converge to a scalar that can be recast as the infimum value of a minimization problem set on the unit cell of the lattice (here the unit cube), the minimizer of which is the limit of the sequences of densities $\rho_{N}$. We recover here a result similar to those of the $\Gamma$-limit theory.

The thermodynamic limit in the terms stated above has been the subject of many outstanding contributions, notably by L. Van Hove, D. Ruelle, or Ch. Fefferman [104], in the second half of the 20th century.

But for the models we deal with in the present paper, the landmark work is that by E. Lieb and B. Simon on the thermodynamic limit in the framework of the Thomas-Fermi model. They proved that the questions (i)-(ii) of the thermodynamic limit problem can be answered positively in the setting of the TF theory. In particular, they set a Thomas-Fermi-type model for crystals. It is to be mentioned however that their strategy of proof relies very much upon Teller's no binding theorem, and therefore, it cannot be carried through the TFW setting. It is kind of a paradoxical fact that the definition of a crystal in the TF theory is possible, while this model does not allow for binding even for the most simple molecule. Mathematically, the proof thoroughly exploits the pointwise relation $\left(\rho_{N}\right)^{2 / 3}=\frac{3}{5 C_{T F}}\left(\Phi_{N}\right)_{+}$provided by the Euler-Lagrange equation (3.54) between, for all $N$, the electronic density $\rho_{N}$ and the effective potential $\Phi_{N}$ (defined in (3.55)). Note in addition that for the TF model for neutral systems, the Lagrange multiplier vanishes and consequently $\Phi_{N} \geq 0$; thus taking the positive part in the right-hand side is unnecessary. As $\Phi_{N}$ is mononotic with respect to $N$ (see Remark 4.3), the property carries through to $\rho_{N}$ and the limit can be determined. This pointwise relation does not hold for the TFW theory: it is replaced by an elliptic PDE, (3.57), linking $\Phi_{N}$ and $\rho_{N}$. A different strategy of proof has thus to be developed (see in particular Remark 6.3).

The two of us, in collaboration with I. Catto have thus devoted a series of works to the Thomas-Fermi-von Weizsäcker model [64 61, giving a complete description of the thermodynamic limit for this model. For the Hartree and Hartree-Focktype models, the fundamental issues (i)-(ii) remain open. The two key difficulties are first that the latter models are not convex (convexity plays a crucial role in the analysis of the TFW model) and second that the number of wavefunctions $\varphi_{i}$ to be dealt with is also growing to infinity (in the TFW model, only the density $\rho$ is relevant). For the $\mathrm{H}$ and HF models, we have only established preliminary results in [65, 63, 62]. In the $\mathrm{H}$ and $\mathrm{HF}$ cases, we need to postulate, in addition to the periodicity of the set of nuclei, the periodicity of the matrix density in the 
limit. The latter is kind of an auxiliary postulate, in view of the former, and at least is a fact that can be established on the basis of experimental observations. This allows us to then identify and study the variational problem obtained in the thermodynamic limit.

6.2. Thomas-Fermi-type models: the complete story. In order to illustrate the difficulty of the problem, let us recall the TFW energy functional. Without changing the notation, we add to the energy functional defined in Section 3.2 the interaction term accounting for the repulsion between the nuclei:

$$
\begin{aligned}
E_{T F W}(\rho)= & C_{W} \int_{\mathbf{R}^{3}}|\nabla \sqrt{\rho}|^{2}+C_{T F} \int_{\mathbf{R}^{3}} \rho^{5 / 3} \\
& \quad-\sum_{\bar{x}_{k} \in \Lambda_{N}} \int_{\mathbf{R}^{3}} \frac{\rho(x)}{\left|x-\bar{x}_{k}\right|}+\frac{1}{2} \int_{\mathbf{R}^{3}} \int_{\mathbf{R}^{3}} \frac{\rho(x) \rho(y)}{|x-y|} d x d y \\
& \quad+\frac{1}{2} \sum_{\bar{x}_{j} \neq \bar{x}_{k} \in \Lambda_{N}} \frac{1}{\left|\bar{x}_{j}-\bar{x}_{k}\right|}
\end{aligned}
$$

and recall that we expect the energy $E_{T F W}\left(\rho_{N}\right)$ at the minimizer $\rho_{N}$ to be asymptotically linear with respect to $N$. Now it is easy to see that each of the last three terms of the electrostatic energy

$$
\begin{aligned}
E_{\Lambda_{N}}^{\text {elec }}(\rho)=-\sum_{\bar{x}_{k} \in \Lambda_{N}} \int_{\mathbf{R}^{3}} \frac{\rho(x)}{\left|x-\bar{x}_{k}\right|} d x & +\frac{1}{2} \iint_{\mathbf{R}^{3} \times \mathbf{R}^{3}} \frac{\rho(x) \rho(y)}{|x-y|} d x d y \\
& +\frac{1}{2} \sum_{\bar{x}_{j} \neq \bar{x}_{k} \in \Lambda_{N}} \frac{1}{\left|\bar{x}_{j}-\bar{x}_{k}\right|}
\end{aligned}
$$

scales as $N^{5 / 3}$. For instance, a vague evaluation as follows:

$$
\begin{aligned}
\sum_{\bar{x}_{j} \neq \bar{x}_{k} \in \Lambda_{N}} \frac{1}{\left|\bar{x}_{j}-\bar{x}_{k}\right|} & \approx \iint_{(\text {Cube of volume } N)^{2}} \frac{d x d y}{|x-y|} \\
& =N^{5 / 3} \iint_{(\text {Unit cube })^{2}} \frac{d x d y}{|x-y|},
\end{aligned}
$$

using a change of variables in the integral, suffices to prove this for the last term.

Therefore, cancellation effects are needed to obtain a linear behaviour of the electrostatic energy $E_{\Lambda_{N}}^{\text {elec }}(\rho)$. A key point in the strategy of the proof is therefore to prove that the electronic density spreads in such a homogeneous way all over the cube of size $N$ so that the effect of the electronic cloud is to screen the electrostatic interaction, thereby making possible the proper scaling law for the energy $E_{\Lambda_{N}}^{e l e c}(\rho)$.

It is actually doable to write down a mathematical proof that follows the above program, working directly on the energy functional and proving that such cancellations do occur. A more powerful approach to the problem that may be applied to more general settings consists of treating the Euler-Lagrange equation rather than the energy functional.

In particular, this proof permits us to treat general geometries for the infinite set of nuclei. The set of nuclei need not be the periodic measure $\sum_{\bar{x}_{k} \in \mathbb{Z}^{3}} \delta\left(\cdot-\bar{x}_{k}\right)$ but needs only to enjoy convenient properties that will be made precise below.

Let us now introduce the periodic minimization problems we will obtain by passing to the thermodynamic limit. We define the following periodic minimization 
problem set on the unit cell $Q$ of the lattice (take $Q=\left[-\frac{1}{2},+\frac{1}{2}\right]^{3}$ for simplicity):

$$
\begin{aligned}
I_{p e r}^{T F W} & =\inf \left\{E_{\text {per }}^{T F W}(\rho) ; \rho \geq 0, \sqrt{\rho} \in H_{\text {per }}^{1}(Q), \int_{Q} \rho=1\right\}, \\
E_{p e r}^{T F W}(\rho) & =C_{W} \int_{Q}|\nabla \sqrt{\rho}|^{2}+C_{T F} \int_{Q} \rho^{5 / 3}-\int_{Q} \rho(x) G(x) d x \\
& +\frac{1}{2} \iint_{Q \times Q} \rho(x) \rho(y) G(x-y) d x d y,
\end{aligned}
$$

where $H_{p e r}^{1}(Q)$ is the subset of $H_{l o c}^{1}\left(\mathbb{R}^{3}\right)$ consisting of functions which satisfy the periodic boundary conditions on the boundary of $Q$. The potential $G$ which appears in the definition (6.6) of the TFW functional is defined, in a unique way, by

$$
-\Delta G=4 \pi\left(-1+\sum_{y \in \mathbf{Z}^{3}} \delta(\cdot-y)\right)
$$

and

$$
\int_{Q} G=0
$$

The main results are collected in the following theorem.

\section{Theorem 6.1. 61 Thermodynamic limit for the TFW model}

In the thermodynamic limit $N \longrightarrow+\infty$, we have

- (i) convergence of the energy per unit volume:

$$
\lim _{N \rightarrow+\infty} \frac{1}{N} I^{T F W}\left(\Lambda_{N}\right)=I_{p e r}^{T F W}+\frac{M}{2},
$$

where $M / 2$ is a universal constant, that depends only on $G$, through

$$
M=\lim _{x \rightarrow 0}\left[G(x)-\frac{1}{|x|}\right],
$$

and which is just a matter of normalization.

- (ii) convergence of the electronic density $\rho_{N}$ to the minimizer $\rho_{\text {per }}$ of $I_{\text {per }}^{T F W}$ in the following senses: $u_{N}=\sqrt{\rho}_{N}$ converges to $u_{\text {per }}=\sqrt{\rho}_{\text {per }}$ strongly in $H_{\text {loc }}^{1}\left(\mathbb{R}^{3}\right) \cap L_{\text {loc }}^{p}\left(\mathbb{R}^{3}\right)$ for all $1 \leq p<+\infty$, uniformly on the compact sets of $\mathbb{R}^{3}$, and

$$
\left\|\rho_{N}-\rho_{p e r}\right\|_{L^{\infty}\left(\Omega_{N}\right)} \longrightarrow 0,
$$

for any sequence of domains $\Omega_{N}$ included in the large cube of size $N$, growing as this cube, and asymptotically far from its boundary (a sequence called an interior domain in statistical mechanics).

We now outline the proof of this theorem. As mentioned above, part (i) of the theorem can be proven by direct techniques on the energy functional and the variational problems. But the most efficient way to proceed is to prove (ii) and next (i) by arguing on the Euler-Lagrange equation of the TFW problem.

Let us recall that the TFW equation reads as stated in (3.57):

$$
-C_{W} \Delta u_{N}-\left(\sum_{\bar{x}_{k} \in \Lambda_{N}} \frac{1}{\left|x-\bar{x}_{k}\right|}\right) u_{N}+\frac{5}{3} C_{T F} \rho_{N}^{2 / 3} u_{N}+\left(\rho_{N} \star \frac{1}{|x|}\right) u_{N}=-\theta_{N} u_{N},
$$


where $u_{N}=\sqrt{\rho}_{N}$. Introducing again the effective potential

$$
\Phi_{N}=\sum_{\bar{x}_{k} \in \Lambda_{N}} \frac{1}{\left|x-\bar{x}_{k}\right|}-\rho_{N} \star \frac{1}{|x|}-\theta_{N}
$$

it can be written in the form of the system

$$
\left\{\begin{array}{l}
-C_{W} \Delta u_{N}+\frac{5}{3} C_{T F} u_{N}^{7 / 3}-\Phi_{N} u_{N}=0, \\
u_{N} \geq 0, \\
-\frac{1}{4 \pi} \Delta \Phi_{N}=\sum_{\bar{x}_{k} \in \Lambda_{N}} \delta\left(\cdot-\bar{x}_{k}\right)-\rho_{N} .
\end{array}\right.
$$

This is the form we shall manipulate henceforth.

The strategy is as follows. First, we establish some a priori bounds on $\rho_{N}$ and $\Phi_{N}$, which allow us to pass to the limit in the Euler-Lagrange system (6.10) and obtain the system (6.12) below for the limits $(u, \Phi)$. The main result that will allow for the proof of Theorem 6.1 is the uniqueness result stated in Lemma 6.2 below. The proof of this latter lemma is the second step. Once this uniqueness is established, it suffices to suppose some additional structure hypothesis on the measure $m$, such as periodicity, to recover (by a straightforward argument) the same structure on the solution $(u, \Phi)$. Finally, the average energy (here the periodic energy) is then found by simply inserting the convergence of $u_{N}$ and $\Phi_{N}$ in all terms of the energy functional (this will not be detailed here). The proof of Theorem 6.1 is then concluded.

Lemma 6.2. Let $m$ be a locally bounded measure that satisfies

$$
\left\{\begin{array}{l}
\sup _{x \in \mathbf{R}} m\left(x+B_{1}\right)<+\infty, \\
\exists R, \inf _{x \in \mathbf{R}^{3}} m\left(x+B_{R}\right) \geq 1 .
\end{array}\right.
$$

Then there is one and only one solution $(u, \Phi)$ to the system

$$
\left\{\begin{array}{l}
-C_{W} \Delta u+\frac{5}{3} C_{T F} u^{7 / 3}-\Phi u=0 \\
u \geq 0 \\
-\frac{1}{4 \pi} \Delta \Phi=m-\rho
\end{array}\right.
$$

such that $u \in L^{\infty}$ and $\Phi$ belongs to $L_{\text {unif }}^{1}\left(\mathbb{R}^{3}\right)$ (which means $\left.\sup _{x \in \mathbb{R}^{3}}\|\Phi\|_{L^{1}\left(x+B_{1}\right)}<+\infty\right)$. In addition,

$$
\inf _{\mathbb{R}^{3}} u>0,
$$

and $\Phi$ belongs to the uniform Marcinkiewitz space $L_{\text {unif }}^{3, \infty}\left(\mathbb{R}^{3}\right)$, i.e.

$$
\sup _{y \in \mathbf{R}^{3}} \sup _{t>0} t^{3} \text { Meas }\left\{x \in y+B_{1} ;|\Phi(x)| \geq t\right\}<+\infty .
$$

Some remarks are needed on assumptions (6.11). Of course the case of the periodic lattice corresponds to $m=\sum_{\bar{x}_{k} \in \mathbb{Z}^{3}} \delta\left(\cdot-\bar{x}_{k}\right)$, which fulfills the above conditions. These conditions are known as those defining a Delaunay lattice in crystallography. Heuristically, Assumptions (6.11) exclude situations where the set of nuclei include infinitely charged clusters of nuclei somewhere or infinitely huge empty zones. We will come back to these assumptions in Section 7.1 below. 
It is worth emphasizing the fundamental reason why uniqueness holds for a system of type (6.12). Basically, the reason is the strict convexity of the TFW energy functional. As indicated by the condition $u \geq 0$, we deal here with the ground state, and this ground state is unique. However, the argument is not as straightforward as the natural framework would be to work in the energy space, say $H^{1}$, or any space of functions that in some weak sense at least vanish at infinity: now the solution we manipulate is definitely not in such a space, as we expect it to be periodic. The point is therefore to understand to which extent there is still enough convexity in the equations to enforce uniqueness even without the "boundary condition" at infinity. In this respect, the argument is reminiscent of arguments of [17] and [4] on the equation

$$
-\Delta \Phi+|\Phi|^{p-1} \Phi=m
$$

with $m$ a locally bounded measure. The latter equation can in fact be recovered as a degenerate case of our system (6.12) by erasing the Laplacian term and formally factoring out by $u_{N}$.

The first step of the proof of Theorem 6.1 is devoted to the derivation of $a$ priori estimates, as usual in such contexts. These a priori estimates translate the fact that $\rho_{N}$ progressively fills in the whole space in a rather regular way, thereby preparing the second step where periodicity is proven. This homogeneity of $\rho_{N}$ in space indeed comes from an a priori estimate on the energy: as the energy is known to be bounded, the cancellation effects mentioned above must occur, and thus $\rho_{N}$ resembles $\sum_{\bar{x}_{k} \in \Lambda_{N}} \delta\left(\cdot-\bar{x}_{k}\right)$. In terms of equations, the effective potential $\Phi_{N}$ is first shown to be bounded, and thus, as

$$
-\frac{1}{4 \pi} \Delta \Phi_{N}=\sum_{\bar{x}_{k} \in \Lambda_{N}} \delta\left(\cdot-\bar{x}_{k}\right)-\rho_{N},
$$

it follows that $\rho_{N}$ is close to the sum of the Dirac masses.

The very first estimate is provided by an inequality which is a general observation due to J.P. Solovej 189:

$$
\alpha u_{N}^{4 / 3}-\Phi_{N} \leq\left(C-\theta_{N}\right)_{+},
$$

for some constant $C$ (independent of $N$ ) and all $\alpha$ small enough. This estimate is based upon a tricky argument and a standard application of the maximum principle for elliptic operators. It provides us with the bound from below

$$
\Phi_{N} \geq C^{t e} \text {. }
$$

Remark 6.3. The estimate (6.13) shows that "half of the" pointwise information of the TF case $\Phi_{N}=C^{t e} u_{N}^{4 / 3}$ survives for the TFW model. More generally, the determination of the thermodynamic limit for the TFW model follows the same pattern as that for the TF model, with the difference that the pointwise relations linking the potential to the density in the vein of the Euler-Lagrange equation (3.54) are now replaced by elliptic PDEs, such as (3.57). The repeated use of the maximum principle allows for circumventing the difficulties while obtaining roughly the same results.

Next we turn to the bound from above for $\Phi_{N}$. This is done first by showing a bound in the average, namely that

$$
\Phi_{N} \star \mu_{\delta} \leq C(\delta)
$$


where $\mu_{\delta}$ is a regularization kernel with parameter $\delta$ and $C(\delta)$ is a constant depending on $\delta$ but not on $N$. Such information comes from the observation along which the first eigenvalue

$$
\lambda_{1}\left(-C_{W} \Delta+\frac{5}{3} C_{T F} u_{N}^{4 / 3}-\Phi_{N}, \Omega\right) \geq 0,
$$

of the operator appearing in the left-hand side of the TFW equation needs to be nonnegative on any bounded domain $\Omega$ (by a simple application of the Green formula, and the Hopf Lemma). Now, and this is a general fact, if we have information on the sign of a Schrödinger operator, we certainly cannot deduce an estimate on its potential, but we can do it on the average of the potential, thus the bound (6.15).

Next, we use the estimates above to prove that the potential itself is bounded from above:

$$
\Phi_{N}(x) \leq C^{t e} \operatorname{Max}_{\bar{x}_{k} \in \Lambda_{N}} \frac{1}{\left|x-\bar{x}_{k}\right|} .
$$

The latter bound is obtained by a technique of comparison on solutions for elliptic equations.

Collecting (6.14) and (6.16), we know that $\Phi_{N}$ remains bounded independently of $N$, which is, as we said, an expression of the fact that cancellation effects do occur.

It now suffices to turn to $u_{N}$. As

$$
-\Delta u_{N}+u_{N}^{7 / 3} \leq \Phi_{N} u_{N}
$$

(up to irrelevant constants), we deduce using Young's inequality in the right-hand side and again comparison techniques on $-\Delta u+u^{p}$ that $u_{N}$ is bounded independently of $N$.

We may therefore pass to the limit in the Euler-Lagrange equation and obtain the system (6.12) for the limits $(u, \Phi)$.

The second part of the proof consists of proving that a solution $(u, \Phi)$ to the Euler-Lagrange equation is periodic. For this purpose, we know it suffices to prove the uniqueness, i.e. Lemma 6.2 A simple argument of translation will then give the periodicity.

We begin by noticing that a (nontrivial) nonnegative solution $u$ is necessarily bounded away from zero, i.e.

$$
\inf _{\mathbb{R}^{3}} u>0
$$

This can be seen by arguing by contradiction. If $u\left(x_{n}\right)$ goes to zero for some sequence of points $x_{n}$, then we may translate and use a compactness argument, so that $x_{n}$ can be supposed to be identically the origin in $\mathbb{R}^{3}$ without loss of generality. Then we repeatedly use the Harnack inequality to prove $u=0$ and reach a contradiction. In such an argument, we crucially use the fact that there is homogeneity (no hole, no cluster) encoded in the periodicity of the lattice.

Once we know that nonnegative solutions satisfy (6.17), we are in a position to conclude the proof.

Let us forget all constants for simplicity, and consider two positive solutions $u$ and $v$ of the equation

$$
\left\{\begin{array}{l}
-\Delta u+u^{7 / 3}-\phi u=0 \\
-\Delta v+v^{7 / 3}-\psi v=0
\end{array}\right.
$$


thus satisfying $\inf _{\mathbb{R}^{3}} u>0$ and $\inf _{\mathbb{R}^{3}} v>0$. Subtracting one equation from the other yields

$$
-\Delta(u-v)+u^{7 / 3}-v^{7 / 3}-(\phi u-\psi v)=0 .
$$

Using the convexity of $t \longrightarrow t^{7 / 3}$ and writing $\phi u-\psi v=\frac{\phi+\psi}{2} w+\frac{\phi-\psi}{2}(u+v)$, we obtain

$$
L w+\frac{4}{3} \frac{u^{4 / 3}+v^{4 / 3}}{2} w-\frac{u+v}{2}(\phi-\psi) \leq 0,
$$

where $w$ denotes $u-v$ and

$$
L=-\Delta+\frac{1}{2}\left(u^{4 / 3}+v^{4 / 3}\right)-\frac{\phi+\psi}{2} .
$$

Next we notice that, because $u>0$ and $v>0$ both solve (6.18), the first eigenvalues of the elliptic operator of the left-hand side of (6.18) are nonnegative:

$$
\left\{\begin{array}{l}
\lambda_{1}\left(-\Delta+u^{4 / 3}-\phi\right) \geq 0 \\
\lambda_{1}\left(-\Delta+v^{4 / 3}-\psi\right) \geq 0
\end{array}\right.
$$

on $\mathbb{R}^{3}$. It follows by sum that $\lambda_{1}(L) \geq 0$, and thus that

$$
\lambda_{1}\left(L+\frac{4}{3} \frac{u^{4 / 3}+v^{4 / 3}}{2}\right) \geq \lambda_{1}(L)+\nu>0
$$

for some $\nu>0$ since $u$ and $v$ are both bounded away from 0 . The situation is thus as follows:

$$
\left\{\begin{array}{l}
L w+\nu w \leq \frac{u+v}{2}(\phi-\psi), \\
-\Delta(\phi-\psi)=-(u+v) w .
\end{array}\right.
$$

Using an adequate sequence of cut-off functions, it can be proven that this implies $w=0$ and thus uniqueness.

As announced above, the rest of the proof of Theorem 6.1] is a simple matter.

6.3. Hartree-type models: partial results. With the notation of this section, emphasizing the dependence with respect to the set of nuclei $\Lambda_{N}$, and that for the electrostatic energy introduced above in (6.4), the Hartree model reads

$$
I_{\Lambda_{N}}^{H}=\inf \left\{E_{\Lambda_{N}}^{H}\left(\varphi_{1} ; \cdots ; \varphi_{N}\right) ; \varphi_{i} \in H^{1}\left(\mathbb{R}^{3}\right), \int_{\mathbf{R}^{3}} \varphi_{i}^{2}=1,1 \leq i \leq N\right\},
$$

where the $\varphi_{i}$ are the atomic orbitals and where

$$
E_{\Lambda_{N}}^{H}\left(\varphi_{1} ; \cdots ; \varphi_{N}\right)=\sum_{i=1}^{N}\left(\int_{\mathbf{R}^{3}}\left|\nabla \varphi_{i}\right|^{2}-\frac{1}{2} D\left(\varphi_{i}^{2}, \varphi_{i}^{2}\right)\right)+E_{\Lambda_{N}}^{\text {elec }}(\rho)
$$

with $\rho=\sum_{i=1}^{N}\left|\varphi_{i}\right|^{2}$, and we recall the notation (3.12).

The sum of the self-interactions of the electrons, that is $\sum_{i=1}^{N} D\left(\varphi_{i}^{2}, \varphi_{i}^{2}\right)$, is expected to be of the order of $N$. As the other electrostatic terms of $E_{\Lambda_{N}}^{\text {elec }}(\rho)$ are of the order of $N^{5 / 3}$, one could be inclined to believe that the term $\sum_{i=1}^{N} D\left(\varphi_{i}^{2}, \varphi_{i}^{2}\right)$ does not contribute to the thermodynamic limit. This is not the case. Indeed, the comparison of two different models will illustrate this point. First the Restricted Hartree 
model may be considered. It is obtained by getting rid of this self-interaction, and reads:

$$
\begin{gathered}
I_{\Lambda_{N}}^{R H}=\inf \left\{E_{\Lambda_{N}}^{R H}\left(\varphi_{1} ; \cdots ; \varphi_{N}\right) ; \varphi_{i} \in H^{1}\left(\mathbb{R}^{3}\right), \int_{\mathbf{R}^{3}} \varphi_{i}^{2}=1,1 \leq i \leq N\right\}, \\
E_{\Lambda_{N}}^{R H}\left(\varphi_{1} ; \cdots ; \varphi_{N}\right)=\sum_{i=1}^{N} \int_{\mathbf{R}^{3}}\left|\nabla \varphi_{i}\right|^{2}+E_{\Lambda_{N}}^{\text {elec }}(\rho) .
\end{gathered}
$$

It turns out that the unique minimizer of $E_{\Lambda_{N}}^{R H}\left(\varphi_{1} ; \cdots ; \varphi_{N}\right)$ is of the form

$$
\left(\frac{\sqrt{\rho_{\Lambda_{N}}}}{N} ; \cdots ; \frac{\sqrt{\rho_{\Lambda_{N}}}}{N}\right) \text {. }
$$

For this model, the limit of the energy per unit volume may be identified as

$$
I_{\text {per }}^{R H}=\inf \left\{E_{\text {per }}^{R H}(\rho) ; \rho \geq 0, \sqrt{\rho} \in H_{\text {per }}^{1}(Q) \int_{Q} \rho=1\right\}+\frac{M}{2},
$$

where

$$
E_{p e r}^{R H}(\rho)=\int_{Q}|\nabla \sqrt{\rho}|^{2}-\int_{Q} G \rho+\frac{1}{2} \iint_{Q \times Q} \rho(x) G(x-y) \rho(y) d x d y .
$$

We thus obtain a model, say of the Thomas-Fermi type, to fix the ideas.

Alternatively, a particular Hartree model may be considered. This model emphasizes the contribution of the self-interaction term. We consider a model where the point nuclei have been smeared out by a convolution with a smooth regularization kernel. On purpose, we choose this kernel as the minimizer $\varphi_{C}$ of the Choquard problem:

$$
I_{C}=\inf \left\{\int_{\mathbb{R}^{3}}|\nabla \varphi|^{2}-\frac{1}{2} D\left(\varphi^{2}, \varphi^{2}\right) ; \varphi \in H^{1}\left(\mathbb{R}^{3}\right), \int_{\mathbb{R}^{3}} \varphi^{2}=1\right\} .
$$

Then it is simple to see that the Hartree model with nuclei smeared out in this way converges in the thermodynamic limit to

$$
\inf \left\{\int_{\mathbb{R}^{3}}|\nabla \varphi|^{2}-\frac{1}{2} D\left(\varphi^{2}, \varphi^{2}\right)+\frac{1}{2} \iint_{Q \times Q}\left(\rho-\varphi_{C}^{2}\right)(x) G(x-y)\left(\rho-\varphi_{C}^{2}\right)(y) d x d y ;\right.
$$

$$
\left.\rho=\sum_{k \in \mathbb{Z}^{3}} \varphi^{2}(\cdot+k), \quad \varphi \in H^{1}\left(\mathbb{R}^{3}\right) \int_{\mathbb{R}^{3}} \varphi^{2}=1\right\}-\frac{1}{2} D\left(\varphi_{C}^{2}, \varphi_{C}^{2}\right),
$$

which indeed is $I_{C}$, thereby proving that the limit is not a functional of the density, and that the self-interaction term does have an impact on the thermodynamic limit.

In view of the above two examples, and in the absence of a definite strategy to prove the thermodynamic limit for the Hartree problem, a reasonable guess is that the problem obtained in the limit reads as follows:

$$
\begin{aligned}
I_{p e r}^{H}=\inf & \left\{E_{\text {per }}^{H}(\varphi) ; \varphi \in H^{1}\left(\mathbb{R}^{3}\right), \int_{\mathbf{R}^{3}} \varphi^{2}=1\right\}+\frac{M}{2}, \\
E_{p e r}^{H}(\varphi)= & \int_{\mathbb{R}^{3}}|\nabla \varphi|^{2}-\frac{1}{2} D\left(\varphi^{2}, \varphi^{2}\right) \\
& -\int_{Q} G \rho+\frac{1}{2} \iint_{Q \times Q} \rho(x) G(x-y) \rho(y) d x d y,
\end{aligned}
$$


with $\rho(x)=\sum_{k \in \mathbf{Z}^{3}} \varphi^{2}(x+k)$.

A precise mathematical analysis of the above problem has been conducted in [63] and has shown that there exists a minimizer.

6.4. Hartree-Fock-type models: partial results again. For the Hartree-Fock model, it is intuitive (also for the reader at this stage of the present survey) that the results will be even harder to obtain than in the Hartree case.

For the mathematical analysis of the thermodynamic limit problem of this model, the convenient language is that of density matrices, as introduced in Section 3.3 above. We now detail a little bit further than above the mathematical context.

The (reduced) one-particle density matrix, denoted by $\gamma$ in the sequel, is a selfadjoint operator on $L^{2}\left(\mathbf{R}^{3}\right)$, with finite trace, such that

$$
0 \leq \gamma \leq 1
$$

(in the sense of self-adjoint operators) where $\mathbf{1}$ denotes the identity on $L^{2}\left(\mathbf{R}^{3}\right)$, and whose trace fits the number of electrons. The density matrix being a HilbertSchmidt operator on $L^{2}\left(\mathbf{R}^{3}\right)$, its kernel (still denoted by $\gamma$ ) may be decomposed along a complete set of orthonormal eigenfunctions $\left(\varphi_{n}\right)_{n \geq 1} \in L^{2}\left(\mathbf{R}^{3}\right)$ of $\gamma$ associated to the eigenvalues $0 \leq \lambda_{n} \leq 1$ in such a way that $\gamma(x, y)=\sum_{n \geq 1} \lambda_{n} \varphi_{n}(x) \varphi_{n}^{\star}(y)$. The density is then $\rho_{\gamma}=\sum_{n>1} \lambda_{n}\left|\varphi_{n}(x)\right|^{2}$. The Hartree-Fock functional may then be expressed in terms of density matrices as

$$
E_{\Lambda_{N}}^{H F}(\gamma)=\operatorname{Tr}[-\Delta \gamma]+E_{\Lambda_{N}}^{\text {elec }}\left(\rho_{\gamma}\right)-\frac{1}{2} \iint_{\mathbf{R}^{3} \times \mathbf{R}^{3}} \frac{|\gamma(x, y)|^{2}}{|x-y|} d x d y,
$$

where $E_{\Lambda_{N}}^{\text {elec }}\left(\rho_{\gamma}\right)$ is defined in (6.4) and thus accounts for the repulsion between nuclei. Let us note that, in the above expression of the energy, we have

$$
\operatorname{Tr}[-\Delta \gamma]=\sum_{n \geq 1} \lambda_{n} \int_{\mathbf{R}^{3}}\left|\nabla \varphi_{n}(x)\right|^{2} d x .
$$

The Hartree-Fock ground-state energy is then defined by

$$
I_{\Lambda_{N}}^{H F}=\inf \left\{E_{\Lambda_{N}}^{H F}(\gamma) ; \gamma \in \Gamma_{N}\right\},
$$

where the set of admissible density matrices $\Gamma_{N}$ is composed of density matrices as above, satisfying in addition $\gamma^{2}=\gamma, \operatorname{Tr}(\gamma)=N$ and that $\operatorname{Tr}[-\Delta \gamma]<+\infty$.

No result to date gives the thermodynamic limit of the HF problem. Nevertheless, there is such a result for the restricted Hartree-Fock model, which consists of erasing the exchange term in the HF model. The analysis is then far simpler because convexity is restored. This model can be shown to converge to the RHF periodic model

$$
\begin{aligned}
& I_{\text {per }}^{R H F}=\inf \left\{E_{\text {per }}^{R H F}\left(\gamma_{\text {per }}\right) ; \gamma_{\text {per }} \in \Gamma\right\}, \\
E_{\text {per }}^{R H F}\left(\gamma_{\text {per }}\right)= & \frac{1}{(2 \pi)^{3}} \int_{Q^{\star}} \operatorname{Tr}_{L_{\xi}^{2}(Q)}\left[-\Delta_{\xi} \gamma_{\xi}\right] d \xi \\
& -\int_{Q} G \rho_{\text {per }}+\frac{1}{2} \iint_{Q \times Q} \rho_{\text {per }}(x) G(x-y) \rho_{\text {per }}(y) d x d y
\end{aligned}
$$

in the thermodynamic limit.

On the basis of this, it is natural to postulate that the Hartree-Fock energy per unit volume converges to the following periodic model:

$$
I_{p e r}^{H F}=\inf \left\{E_{p e r}^{H F}\left(\gamma_{p e r}\right) ; \gamma_{p e r} \in \Gamma\right\}
$$




$$
\begin{aligned}
& E_{p e r}^{H F}\left(\gamma_{p e r}\right)=\frac{1}{(2 \pi)^{3}} \int_{Q^{\star}} \operatorname{Tr}_{L_{\xi}^{2}(Q)}\left[-\Delta_{\xi} \gamma_{\xi}\right] d \xi-\int_{Q} G \rho_{p e r} \\
& \quad+\frac{1}{2} \iint_{Q \times Q} \rho_{p e r}(x) G(x-y) \rho_{p e r}(y) d x d y-\frac{1}{2} \iint_{Q \times \mathbf{R}^{3}} \frac{\left|\gamma_{p e r}(x, y)\right|^{2}}{|x-y|} d x d y .
\end{aligned}
$$

This model can be shown to be well-posed (see [62]): there exists a minimizer. It is worth noticing that the exchange term may be rewritten in the equivalent form

$$
\begin{aligned}
& \iint_{Q \times \mathbf{R}^{3}} \frac{\left|\gamma_{p e r}(x, y)\right|^{2}}{|x-y|} d x d y \\
& \quad=\frac{1}{(2 \pi)^{6}} \iiint \int_{\left(Q^{\star}\right)^{2} \times Q^{2}} \gamma_{\xi}(x, y) W_{\infty}\left(\xi-\xi^{\prime}, x-y\right) \gamma_{\xi^{\prime}}(x, y)^{*} d \xi d \xi^{\prime} d x d y
\end{aligned}
$$

where, for every $\eta$ in $Q^{\star}$ and $z$ in $\mathbb{R}^{3}$,

$$
W_{\infty}(\eta, z)=\sum_{k \in \mathbf{Z}^{3}} \frac{e^{i k \cdot \eta}}{|z+k|} .
$$

(Note that $e^{i \eta \cdot z} W_{\infty}(\eta, z)$ is $Q$-periodic with respect to $z$.) On this latter form the nonlocal feature of the exchange term is more easily seen. The Euler-Lagrange equation satisfied by any minimizer may be written as follows: at every fixed phase $\xi$ in $Q^{\star}$, and for every $n \geq 1$,

$$
\begin{gathered}
(i \nabla+\xi)^{2} u_{n}(\xi, \cdot)-G u_{n}(\xi, \cdot)+\left(\int_{Q} G(\cdot-y) \rho_{\xi}(y) d y\right) u_{n}(\xi, \cdot) \\
-\frac{1}{(2 \pi)^{3}} \iint_{Q^{\star} \times Q} \gamma\left(\xi^{\prime} ; \cdot ; y\right) W_{\infty}\left(\xi-\xi^{\prime} ; \cdot-y\right) e^{i \xi \cdot y} u_{n}(\xi, y) d y d \xi^{\prime} \\
=\varepsilon_{n}(\xi) u_{n}(\xi, \cdot), \quad \text { on } Q,
\end{gathered}
$$

together with

$$
\left\{\begin{array}{l}
\lambda_{n}(\xi)=0 \Longrightarrow \varepsilon_{n}(\xi) \geq \pi \\
0<\lambda_{n}(\xi)<1 \Longrightarrow \varepsilon_{n}(\xi)=\pi \\
\lambda_{n}(\xi)=1 \Longrightarrow \varepsilon_{n}(\xi) \leq \pi .
\end{array}\right.
$$

These equations are indeed those solved in the numerical simulations.

\section{Remark 6.4. On the relativistic models for the solid phase}

It is possible to examine the same questions as those addressed here in the context of relativistic models. A recent work [60] aims to define in a rigorous way a Dirac-Fock model for crystals.

\section{UNADDRESSED TOPICS, TRENDS AND PERSPECTIVES}

We collect in this final section some topics that have been hardly addressed so far in this article and that we consider to be interesting approaches for further research. To date, we have only obtained preliminary results in the directions that are mentioned below. We hope to be able to complement these results in the near future. 
7.1. Energy of infinite microscopic systems. We have made it clear from the very beginning of Section [6 that, in the absence of a theoretical solution of the crystal problem, the results enclosed therein were demonstrating, in good cases, the periodicity of the electronic structure, being postulated that the nuclei arrange themselves periodically. The enterprise of trying to establish the periodicity of the nuclei has been mentioned to be a difficult approach. At this stage, many ways for proceeding further may be envisioned.

The main approach we would like to follow is to ask the following question. Suppose we are definitely not able to prove the periodicity of the set of nuclei, or suppose that this periodicity simply does not hold. Would we be able to still define the energy for the infinite set of nuclei and electrons within a given model? Slightly changing the viewpoint is indeed worthwhile. The following question may thus be asked:

What is the most general geometric property of an infinite set of nuclei

(possibly accompanied by their cloud of electrons) that allows us to define

the average energy of the set?

Let us first lay some groundwork. There are simple extensions of periodicity that allow for a straightforward generalization of the arguments of Section6. Indeed, as the heart of the matter is the existence and uniqueness Lemma 6.2 and the assumptions (6.11) therein, it suffices to say that these assumptions hold for quasiperiodic and almost periodic measures, and consequently obtain a result of thermodynamic limit for quasicrystals. Such extensions are included in [61]. However, these extensions are to some extent too close to periodicity, and might be judged unsatisfactory, with a view to seeking generality.

An interesting point to make is that, if the assumptions (6.11) are in some sense important, they are not absolutely necessary either, and can be slightly circumvented. Indeed, one may examine the case of a periodic one-dimensional structure embedded in $\mathbb{R}^{3}$ (a model for a linear very large molecule), or that of a periodic two-dimensional structure embedded again in $\mathbb{R}^{3}$ (a model for a thin film), and still prove the existence of a limit energy (see [24]). Such situations obviously do not satisfy the assumptions (6.11). In both of the above situations, these assumptions are nevertheless fulfilled "along the direction of" the infinite part of the system, which is the only necessary property needed. Apart from its interest per se, the study of such models arises as the need to rule out degenerate situations when proving the existence of an optimal periodic lattice that minimizes the periodic energy among all possible periodic lattices; see 21, 22. Another, and much more important, example of a situation that can be treated with the same tools as the full crystal is the case of surface energies, i.e. the definition of the energy of a crystal filling in the half space $x_{1}<0$ in $\mathbb{R}^{3}$, while the other half is a vacuum; see [23]. One step beyond the definition of surface energies is that of interface energies, an approach that will be followed in the near future. Likewise, the definition of the energy of defaults in crystals, i.e. mathematically compact perturbations of periodic structures, can be examined.

More generally, a possible answer to question (7.1) has been provided in [27]. Based on some very weak properties imposed to the set of nuclei (these properties in particular include assumptions (6.11)), we are able to construct an algebra of functions, on which we set the variational problems that express the energy of 
the infinite set of particles. We currently examine an alternative way that relies upon the introduction of a stochastic formalism. The positions of the nuclei are now random variables, or alternatively the measure $m$ defining these positions is a stationary random process $m(\cdot, \omega)$. It is natural, and it actually underlies the results proven so far, that the notion of stationary process plays a role in such a problem. What is indeed required is that, whatever the place it is located, the nucleus sees the same environment. A simple way to think of such a formalism is to consider a random perturbation of periodic lattices, but the formalism is indeed much more general than that. Then, energies can be defined in terms of expectation values. Again, Assumptions (6.11) are not stricto sensu needed. We refer to [28, still in progress.

7.2. Macroscopic limits. It is a longstanding goal to determine densities of continuum mechanical energy on the basis of microscopic models for matter. Mathematically, this amounts to determining the macroscopic limits of our microscopic energies for infinite sets, when the material is subjected to given forces that induce a deformation. For instance, determining the macroscopic limit of microscopic lattices energies has been often performed by Gamma-limit techniques. We refer e.g. to [75, 42, 43, and the references therein. The works [44, 45, 46, 177, 116] are instances of works that more precisely deal with the macroscopic limit of discrete systems. We also would like to mention the work [115]. In [29] and [26], we have investigated a strategy consisting of passing to the macroscopic limit for a given macroscopic deformation of the sample. This has been done in the context of perfect crystals and a given fixed deformation. The various extensions described in the previous section could allow for a generalization of this procedure.

A better understanding at the theoretical level of the connections between the discrete microscopic description of matter with the continuous macroscopic description would certainly have a deep impact on the numerical simulations commonly used in materials science. This is true first in a static picture, which has been the main viewpoint adopted in the present survey, but also in a dynamic picture, when trying to relate/combine molecular dynamics simulation and dynamic simulation in continuum mechanics (propagation of fractures, etc...). Such theoretical contributions would open the way to a rigorous foundation of the methods of multiscale simulations of materials (see e.g. [183, 78, 170]).

\section{ACKNOWLEDGEMENTS}

The authors would like to thank X. Blanc, E. Cancès, I. Catto, and M. J. Esteban for a critical reading of a preliminary version of the present article.

\section{REFERENCES}

1. Allen, M.P. and D.J. Tildesley (1987) Computer simulation of liquids (Oxford Science Publications).

2. Ashcroft, N.W. and N. D. Mermin (1976) Solid-State Physics (Saunders College Publishing).

3. Auchmuty, G. and Wenyao Jia (1994) Convergent iterative methods for the Hartree eigenproblem, Math. Model. and Num. Anal. 28, pp. 575-610. MR1295588 (95m:81230)

4. Bach, V., E.H. Lieb, M. Loss and J.P. Solovej (1994) There are no unfilled shells in unrestricted Hartree-Fock theory, Phys. Rev. Lett. 72, pp. 2981-2983.

5. BACH, V. (1992) Error bound for the Hartree-Fock energy of atoms and molecules, Comm. Math. Phys. 147, pp. 527-548. MR1175492 (94d:81242) 
6. BACSkAY, G.B. (1961) A quadratically convergent Hartree-Fock (QC-SCF) method. Application to closed shell systems, Chem. Phys. 61, pp. 385-404.

7. BaLÀzs, N. (1967) Formation of stable molecules within statistical theory of atoms, Phys. Rev., vol. 156, pp. 42-47.

8. BAlian, R. (1991) From microphysics to macrophysics: Methods and applications of statistical physics, in 2 volumes, Springer. MR.1129462 (92g:82001)

9. Bardos, C., F. Golse, A. Gottlieb, N. Mauser (2003) Mean-field dynamics of fermions and the time-dependent Hartree-Fock equation, JMPA 82, 6, pp. 665-683. MR 1996777 (2004f:82051)

10. Bandrauk, A., M. Delfour and C. Le Bris, eds. (2004) Quantum control: mathematical and numerical challenges, American Mathematical Society, CRM proceedings, vol. 33. MR2043517 (2004j:00023)

11. Baudouin, L., O. Kavian, J.-P. Puel (2003) Régularité dans une équation de Schrödinger avec potentiel singulier à distance finie et à l'infini (Regularity in a Schrödinger equation with a potential singular at finite distance and at infinity), Comptes Rendus Mathématique, vol. 337, 11, pp. 705-710. MR2030406 (2004k:35308)

12. Baudouin, L., O. Kavian, J.-P. Puel (2005) Regularity for a Schrödinger equation with a potential singular at finite distance and at infinity and application to a bilinear optimal control problem, to appear.

13. Baudouin, L., J.-P. Puel (2005), in preparation.

14. Benguria, R., H. BrÉzis and E.H. Lieb (1981) The Thomas-Fermi-von Weizsäcker theory of atoms and molecules, Comm. Math. Phys. 79, pp. 167-180. MR0612246 (83m:81114)

15. Benguria, R. and E.H. Lieb (1985) The most negative ion in the Thomas-Fermi-von Weizsäcker theory of atoms and molecules, J. Phys. B 18, pp. 1045-1059. MR0786999 (86h:81138)

16. Benguria, R. and C. Yarur (1990) Sharp condition on the decay of the potential for the absence of a zero-energy ground state of the Schrödinger equation, J. Phys. A 23, pp. 15131518. MR.1048781 (91d:81020)

17. Benilan, Ph., H. Brézis and M. Crandall (1975) A semilinear equation in $L^{1}\left(\mathbb{R}^{N}\right)$, Ann. Scuola. Norm. Pisa 2, pp. 523-555. MF0390473 (52:11299)

18. Benilan, Ph., J.A. Goldstein and G.R. Rieder (1991) The Fermi-Amaldi correction in spin polarized Thomas-Fermi theory, in: C. Bennewitz, ed., Differential equations and mathematical physics (Academic Press), pp. 25-37. MR1126688 (92m:81309)

19. Benilan, Ph., J.A. Goldstein and G.R. Rieder (1992) A nonlinear elliptic system arising in electron density theory, Comm. Part. Diff. Equ. 17, pp. 2079-2092. MR 1194750 (93i:35048)

20. Blanc, X. (2000) A mathematical insight into ab initio simulations of solid phase, in: M. Defranceschi, C. Le Bris, eds., Mathematical Models and Methods for Ab Initio Quantum Chemistry, Lecture Notes in Chemistry 74 (Springer), pp. 133-158. MR 1855578

21. Blanc, X. (2001) Geometry optimization for crystals in Thomas-Fermi type theories of solids, Comm. P.D.E. 26, 3-4, pp. 651-696. MR1842045 (2002f:82035)

22. Blanc, X. and C. Le Bris (1999) Optimisation de géométrie dans le cadre des théories de and type Thomas-Fermi pour les cristaux périodiques, Note aux Comptes Rendus de l'Académie des Sciences, t. 329, Série 1, pp. 551-556. MR1715124 (2000g:82029)

23. Blanc, X. and C. Le Bris (2005), Définition d'energies d'interface à partir de modèles atomiques, C. R. Acad. Sci., Série I, in press.

24. Blanc, X. and C. Le Bris (2000) Thomas-Fermi type theories for polymers and thin films, Advances in Differential Equations 5 (7-9), pp. 977-1032. MR 1776347 (2001h:35153)

25. Blanc, X. and C. Le Bris (2002) Periodicity of the infinite-volume ground state of a onedimensional quantum model, Nonlinear Analysis, Theory, Methods, and Applications 48 (6), pp. 791-803. MR.1879075 (2003c:82088)

26. Blanc, X., C. Le Bris and P.-L. Lions (2002) From molecular models to continuum mechanics, Archives for Rational Mechanics and Analysis, volume 164, pp. 341-381. MR 1933632 (2003i:74004)

27. Blanc, X., C. Le Bris and P.-L. Lions (2003) A definition of the ground state energy for systems composed of infinitely many particles, Communications in P.D.E, Vol. 28, nos. 1-2, pp. 439-475. MR.1974463 (2004g:81073)

28. Blanc, X., C. Le Bris and P.-L. LiOns (2005) On the energy of microscopic infinite stochastic lattices and their macroscopic limits, in preparation. 
29. Blanc, X., C. Le Bris and P.-L. Lions (2001) Convergence de modèles moléculaires vers des modèles de mécanique des milieux continus, Note aux Comptes Rendus de l'Académie des Sciences, t. 332, Série 1, pp. 949-956. MR1838776 (2002a:74034)

30. Blanc, X., C. Le Bris and P.-L. Lions (2002) Caractérisation des fonctions de $\mathbf{R}^{3}$ à potentiel newtonien borné, Note aux Comptes Rendus de l'Académie des Sciences, t. 334, Série 1, pp. 15-21. MR $1888656(2002 \mathrm{~m}: 35038)$

31. BlanchARD, Ph. and E. BRÜNING (1992) Variational methods in mathematical physics (Springer). MR1230382 (95b:58049)

32. Blanchard, Ph. and E. BRÜNING (2002) Mathematical methods in physics. Distributions, Hilbert space operators, and variational methods, Progress in Mathematical Physics 26, Birkhäuser. MR,1936762 (2004c:46001)

33. Bokanowski, O.M. and B. Grebert (1996a) A decomposition theorem for wave functions in molecular quantum chemistry, Math. Mod. and Meth. in App. Sci. 6, pp. 437-466. MR:1395812 (97d:81216)

34. BoKANOWSKI, O.M. and B. Grebert (1996b) Deformations of density functions in molecular quantum chemistry, J. Math. Phys. 37, pp. 1553-1557. MR.1380859 (97j:81399)

35. Bokanowski, O.M. and B. Grebert (1998) Utilization of deformations in molecular quantum chemistry and application to density functional theory, Int. J. Quant. Chem. 68, pp. $221-231$.

36. Bornemann, F.A., P. Nettersheim and Ch. Schütte (1996) Quantum-classical molecular dynamics as an approximation to full quantum dynamics, J. Chem. Phys. 105, pp. 1074-1083.

37. Bornemann, F.A. and Ch. Schütte (1998) A mathematical investigation of the CarParrinello method, Numer. Math. 78, pp. 359-376. MR1603342 (99d:81183)

38. Bornemann, F.A. (1998) Homogenization in time of singularly perturbed mechanical systems, Lectures Notes in Mathematics 1697, Springer. MR1637077 (99g:34105)

39. Bowler, D. et al. (1997) A comparison of linear scaling tight-binding methods, Model. Simul. Mater. Sci. Eng. 5, pp. 199-202.

40. Bowler, D. and M. Gillan (1999) Density matrices in $O(N)$ electronic structure calculations, Comp. Phys. Comm. 120, pp. 95-108.

41. Boys, S.F. (1950) Electronic wavefunctions. I. A general method of calculation for the stationary states of any molecular system, Proc. Roy. Soc. London Ser. A 200, pp. 542-554.

42. Braides, A. and M.S. Gelli (2000) From Discrete to Continuum: A Variational Approach, Lecture Notes SISSA, Trieste.

43. BRAides, A. (2001) From discrete to continuous variational problems: an introduction, Lecture Notes School on Homogenization Techniques and Asymptotic Methods for Problems with Multiple Scales, Torino.

44. Braides, A. (2000) Non-local variational limits of discrete systems, Comm. Contemporary Math. 2, pp. 285-297. MR1759792 (2001h:49021)

45. Braides, A. and M.S. GelLI (2002) Limits of discrete systems with long-range interactions, J. Convex Anal. 9, no. 2, pp. 363-399. MR1970562 (2004c:49026)

46. Braides, A. and M.S. Gelli (2002) Continuum limits of discrete systems without convexity hypotheses, Math. Mech. Solids 7, no. 1, pp. 41-66. MR1900933 (2003c:49014)

47. BrÉzis, H. (1984) Semilinear equations in $\mathbb{R}^{n}$ without condition at infinity, App. Math. \& Opt. 12, pp. 271-282. MR0768633 (86f:35076)

48. Cancès, E., M. Defranceschi, W. KutzelnigG, C. Le Bris and Y. Maday (2003) Computational Quantum Chemistry: A Primer, in Handbook of Numerical Analysis, Special Volume, Computational Chemistry, volume X, North-Holland. MR2008386

49. CANCÈS, E. (2001a) SCF algorithms for Kohn-Sham models with fractional occupation numbers, J. Chem. Phys. 114, pp. 10616-10623.

50. CANCÈs, E. (2001b) SCF algorithms for Hartree-Fock electronic calculations, in: M. Defranceschi and C. Le Bris, eds., Mathematical models and methods for ab initio quantum chemistry, Lecture Notes in Chemistry 74 (Springer), pp. 17-43.

51. CANCÈs, E. and C. LE Bris (1998) On the perturbation methods for some nonlinear Quantum Chemistry models, Math. Mod. and Meth. in App. Sci. 8, pp. 55-94. MF 1612003 (99b:81284)

52. CANCÈs, E. and C. LE BRIS (1999) On the time-dependent Hartree-Fock equations coupled with a classical nuclear dynamics, Math. Mod. and Meth. in App. Sci. 9, pp. 963-990. MR 1710271 (2000j:81297) 
53. CANCÈs, E. and C. LE BRIS (2000a) On the convergence of SCF algorithms for the HartreeFock equations, Math. Model. Num. Anal. 34, pp. 749-774. MR1784484 (2001k:65178)

54. CAncÈs, E. and C. LE BRIS (2000b) Can we outperform the DIIS approach for electronic structure calculations, Int. J. Quantum Chem. 79, pp. 82-90.

55. CAncÈs, E., C. Le Bris and M. Pilot (2000) Contrôle optimal bilinéaire d'une équation de Schrödinger, Note aux Comptes Rendus de l'Académie des Sciences, Série I 330, pp. 567-571. MR1760440 (2001b:49030)

56. Cancès, E., C. Le Bris, B. MennucCi and J. Tomasi (1999) Integral equation methods for molecular scale calculations in the liquid phase, Math. Mod. and Meth. in App. Sci. 9, pp. 35-44. MR 1671531 (99j:92030)

57. Cancès, E., B. Jourdain and T. Lelièvre (2005), Quantum Monte-Carlo simulations of fermions: a mathematical analysis of the fixed-node approximation, submitted to Math. Models Methods Appl. Sci.

58. CAR, R. and M. PARrinello (1985) Unified approach for molecular dynamics and density functional theory, Phys. Rev. Lett. 55, pp. 2471-2474.

59. Carter, E. and A. Wang (2000) Orbital-Free Kinetic Energy Density Functional Theory, in Theoretical Methods in Condensed Phase Chemistry, S. D. Schwartz, ed., Progress in Theoretical Chemistry and Physics, Kluwer, pp. 117-184.

60. Catto, I. and E. Paturel, in preparation.

61. Catto, I., C. Le Bris and P.-L. Lions (1998) Mathematical theory of thermodynamic limits: Thomas-Fermi type models (Oxford University Press). MR 1673212 (2000e:81002)

62. Catto, I., C. Le Bris and P.-L. Lions (2001) On the thermodynamic limit for Hartree-Fock type models, Annales de l'Institut Henri Poincaré, Analyse non linéaire 18, pp. 687-760. MR.1860952 (2003e:81044a)

63. Catto, I., C. Le Bris and P.-L. Lions (2002) On some periodic Hartree-type models for crystals, Annales de l'Institut Henri Poincaré, Analyse non linéaire 19, pp. 143-190. MR 1902742 (2003e:81044b)

64. CAtto, I., C. Le Bris and P.-L. Lions (1996) Limite thermodynamique pour des modèles de type Thomas-Fermi, Note aux Comptes Rendus de l'Académie des Sciences, t. 322, Série 1, pp. 357-364. MR.1378513 (97b:81149)

65. CAtto, I., C. LE BRIS and P.-L. Lions (1998) Sur la limite thermodynamique pour des modèles de type Hartree et Hartree-Fock, Note aux Comptes Rendus de l'Académie des Sciences, t. 327, Série 1, pp. 259-266. MR.1650265 (99j:81208)

66. Catto, I. and P.-L. Lions (1992) Binding of atoms and stability of molecules in Hartree and Thomas-Fermi type theories, Part I: A necessary and sufficient condition for the stability of general molecular systems, Comm. Part. Diff. Equ. 17, pp. 1051-1110. MR1179279 (94b:81150a)

67. CAtTo, I. and P.-L. Lions (1993a) Binding of atoms and stability of molecules in Hartree and Thomas-Fermi type theories, Part 2: Stability is equivalent to the binding of neutral subsystems, Comm. Part. Diff. Equ. 17, pp. 305-354. MR1211736 (94b:81150b)

68. Catto, I. and P.-L. Lions (1993b) Binding of atoms and stability of molecules in Hartree and Thomas-Fermi type theories, Part 3: Binding of neutral subsystems, Comm. Part. Diff. Equ. 18, pp. 381-429. MR.1214866 (94b:81150c)

69. Catto, I. and P.-L. Lions (1993c) Binding of atoms and stability of molecules in Hartree and Thomas-Fermi type theories, Part 4: Binding of neutral systems for the Hartree model, Comm. Part. Diff. Equ. 18, pp. 1149-1159. MR1233188 (94g:81227)

70. Chadam, J.M. and R.T. Glassey (1975) Global existence of solutions to the Cauchy problem for time-dependent Hartree equations, J. Math. Phys. 16, pp. 1122-1130. MR0413843 $(54: 1957)$

71. Chan, G. K. L. et al. (2001) Thomas-Fermi-Dirac-von Weizsäcker models in finite systems, J. Chem. Phys., vol. 114, 2, pp. 631-638.

72. Challacombe, M. (2000) Linear scaling computation of the Fock matrix. V. Hierarchical cubature for numerical integration of the exchange-correlation matrix, J. Chem. Phys. 113, pp. 10037-10043.

73. Coleman, A. J. and V.I. Yukalov (2000) Reduced density matrices, Lecture Notes in Chemistry 72 (Springer). MR,1757452 (2001k:81376) 
74. Cycon, H.L., R.G. Froese, W. Kirsch and B. Simon (1987) Schrödinger operators with applications to quantum mechanics and global geometry (Springer, New York). MR:0883643 (88g:35003)

75. Dal Maso, G. (1993) An introduction to Gamma-convergence, Progress in Nonlinear Differential Equations and their Applications 8, Birkhäuser Boston. MR1201152 (94a:49001)

76. Daniels, A. and G. Scuseria (1999) What is the best alternative to diagonalization of the hamiltonian in large scale semiempirical calculations?, J. Chem. Phys. 110, pp. 1321-1328.

77. Datta, S.N. and G. Deviah (1988) The minimax technique in relativistic Hartree-Fock calculations, Pramana 30, 5, pp. 393-416.

78. Deák, P., Th. Frauenheim and M. R. Pederson, eds. (2000) Computer simulation of materials at atomic level, Wiley, 2000.

79. Defranceschi, M. and P. Fischer (1998) Numerical solution of the Schrödinger equation in a wavelet basis for hydrogen-like atoms, SIAM J. Num. Anal. 35, pp. 1-12. MR1618416

80. Defranceschi, M. and C. Le Bris (1997) Computing a molecule: a mathematical viewpoint, J. Math. Chem. 21, pp. 1-30. MR1478056

81. Defranceschi, M. and C. Le Bris (1999) Computing a molecule in its environment: a mathematical viewpoint, Int. J. Quant. Chem. 71, pp. 257-250.

82. Defranceschi, M. and C. Le Bris, eds. (2001) Mathematical models and methods for ab initio quantum chemistry, Lecture Notes in Chemistry 74 (Springer). MR 1857459 (2003e:82002)

83. Desclaux, J.P., J. Dolbeault, P. Indelicato, M.J. Esteban and E. Séré (2003) Computational approaches of relativistic models in quantum chemistry, Handbook of Numerical Analysis X (Special Volume on Computational Chemistry), P.G. Ciarlet and C. Le Bris, eds., Elsevier. MR,2008389

84. Dolbeault, J., M.J. Esteban, E. Séré and M. Vanbreugel (2000) Minimization methods for the one-particle Dirac equation, Phys. Rev. Letters 85 (19), pp. 4020-4023.

85. Dolbeault, J., M.J. Esteban and E. SÉRÉ (2000) Variational characterization for eigenvalues of Dirac operators, Cal. Var. 10, pp. 321-347. MR.1767717 (2001f:49083)

86. Dolbeault, J., M.J. Esteban and E. Séré (2000) On the eigenvalues of operators with gaps. Application to Dirac operators, J. Funct. Anal. 174, pp. 208-226. MR 1761368 (2001e:47040)

87. Dolbeault, J., M.J. Esteban and E. SÉré (2000) Variational methods in relativistic quantum mechanics: new approach to the computation of Dirac eigenvalues, in Mathematical Models and Methods for Ab Initio Quantum Chemistry, Lecture Notes in Chemistry, C. Le Bris and M. Defranceschi, eds., Springer, Berlin-Heidelberg. MR.1855581

88. Dolbeault, J., M.J. Esteban and E. SÉré (2003) A variational method for relativistic computations in atomic and molecular physics, Int. J. Quantum. Chemistry 93, pp. 149-155.

89. Dolbeault, J., M.J. Esteban, M. Loss and L. Vega (2004) An analytical proof of Hardylike inequalities related to the Dirac operator, J. Funct. Anal. 216, pp. 1-21. MR2091354

90. Dovesi R., R. Orlando, C. Roetti, C. Pisani and V.R. Saunders (2000) The periodic Hartree-Fock method and its implementation in the crystal code, Phys. Stat. Sol. (b) 217, pp. $63-88$.

91. Dreizler, R.M. and E.K.U. Gross (1990) Density functional theory (Springer).

92. Esteban, M.J. and E. SÉrÉ (1995) Stationary states of the nonlinear Dirac equation, Comm. Math. Phys., vol. 171 (1995), pp. 323-350. Mr.1344729 (96g:81041)

93. Esteban, M.J. and E. SÉRÉ (1994) Existence de solutions stationnaires pour l'équation de Dirac non linéaire et le système de Dirac-Poisson, C.R.A.S. 319, série I, pp. 1213-1218. MR.1309103 (96b:81027)

94. Esteban, M.J., V. Georgiev and E. SÉré (1996) Stationary solutions of the Maxwell-Dirac and the Klein-Gordon-Dirac equations, Cal. Var. 4, pp. 265-281. MF1386737 (97g:35144)

95. Esteban, M.J. and E. SÉRÉ (1997) Existence and multiplicity of solutions for linear and nonlinear Dirac problems, in Partial Differential Equations and Their Applications. P. C. Greiner, V. Ivrii, L. A. Seco and C. Sulem, eds., AMS. MR1479240 (98h:35200)

96. Esteban, M.J., V. Georgiev and E. Séré (1996) Bound-State Solutions of the MaxwellDirac and the Klein-Gordon-Dirac Systems, Lett. Math Phys. 38, pp. 217-220. MR 1403074 (97e:81018)

97. Esteban, M.J. and E. SÉRÉ (1999) Solutions of the Dirac-Fock equations for atoms and molecules, Comm. Math. Phys. 203, pp. 499-530. MR1700174 (2000j:81057) 
98. Esteban, M.J. and E. SÉRÉ (2002) An overview on linear and nonlinear Dirac equations, Discrete and Continuous Dynamical Systems 8 (2), pp. 381-397. MR1897689 (2003d:35219)

99. Esteban, M.J. and E. SÉré (1998) Les équations de Dirac-Fock, Séminaire E.D.P., École Polytechnique, 1997-1998. MR1660518

100. Esteban, M.J. and E. Séré (2001) Nonrelativistic limit of the Dirac-Fock equations, Ann. H. Poincaré 2, pp. 941-961. MR1869528(2003d:81066)

101. Esteban, M.J. and E. SÉRÉ (2002) On some linear and nonlinear eigenvalue problems in relativistic quantum chemistry, Progr. Nonlinear Differential Equations (in A. Marino's honor), Birkhäuser. MR:1879732 (2002j:81060)

102. Esteban, M.J. and E. SÉRÉ (2002) A max-min principle for the ground state of the DiracFock functional, Contemp. Math. 307, pp. 135-141. MR.1946024 (2003k:81052)

103. Esteban, M.J. and E. SÉRÉ, Dirac-Fock models for atoms and molecules and related topics. Proceedings ICMP2003.

104. Fefferman, Ch. (1985) The atomic and molecular nature of matter, Rev. Mat. Iberoamericana 1, pp. 1-44. MR0834355 (88g:82011)

105. Fefferman, Ch. (1986) The N-body problem in quantum mechanics, Commun. Pure Appl. Math. 39, Suppl., S67-S109. MF 0861484 (88e:81171a)

106. Fefferman, Ch. and L.A. Seco (1990) On the energy of a large atom, Bull. A.M.S. 23, 2, pp. 525-530. MR.1056556 (92a:81230)

107. Fefferman, Ch. and L.A. Seco (1994) On the Dirac and Schwinger corrections to the ground-state energy of an atom, Adv. Math. 107, 1, pp. 1-185. MR1283205 (95e:81262)

108. Fefferman, Ch. and L.A. Seco (1995) The mathematics of large atoms, J. Equations Dérivées Partielles, St. Jean de Monts. MR1360480

109. Fischer, P. and M. Defranceschi (1994) The wavelet transform: a new mathematical tool for Quantum Chemistry, in: E.S. Kryachko and J.L. Calais, eds., Conceptual Trends in Quantum Chemistry (Kluwer), pp. 227-247.

110. Fournais, S., M. Hoffmann-Ostenhof, T. Hoffmann-Ostenhof and T. Sorensen (2004) Analyticity of the density of electronic wavefunctions, Ark. Mat. 42, no. 1, pp. 87-106. MR 2056546

111. Fournais, S., M. Hoffmann-Ostenhof, T. Hoffmann-Ostenhof and T. Sorensen (2002) On the regularity of the density of electronic wavefunctions. Mathematical results in quantum mechanics (Taxco, 2001), pp. 143-148, Contemp. Math. 307, Amer. Math. Soc., Providence, RI. MR.1946025 (2003j:81256)

112. Fournais, S., M. Hoffmann-Ostenhof, T. Hoffmann-Ostenhof and T. Sorensen (2002) The electron density is smooth away from the nuclei, Comm. Math. Phys. 228, no. 3, pp. 401-415. MR1918782 (2003f:81287)

113. Frenkel, D. and B. Smit (1996) Understanding molecular simulation (Academic Press).

114. Friesecke, G. (2003) The multiconfiguration equations for atoms and molecules: charge quantization and existence of solutions, Arch. Rat. Mech. Analysis. 169, pp. 35-71. MR $1996268 \mid(2004 \mathrm{~g}: 81315)$

115. Friesecke, G. and R. D. James (2000) A scheme for the passage from atomic to continuum theory for thin films, nanotubes and nanorods, J. Mech. Phys. Solids 48, nos. 6-7, pp. 15191540. MR1766412 (2001c:74007)

116. Friesecke, G. and F. Theil (2002) Validity and failure of the Cauchy-Born hypothesis in a two-dimensional mass-spring lattice, J. Nonlinear Sci. 12, no. 5, pp. 445-478. MR1923388 (2004b:74006)

117. GALLI, G. (2000) Large scale electronic structure calculations using linear scaling methods, Phys. Stat. Sol (b) 217, pp. 231-249.

118. Gardner, C. S. and C. Radin (1979) The infinite-volume ground state of the Lennard-Jones potential, J. Stat. Phys., vol. 20, 6, pp. 719-724. MR0537267(82b:82062)

119. Goedecker, S. (1999) Linear scaling electronic structure methods, Reviews of Modern Physics 71, pp. 1085-1123.

120. Gogny, D. and P.-L. Lions (1986) Hartree-Fock theory in nuclear physics RAIRO, Modélisation Math. Anal. Numer. 20, pp. 571-637. MR0877058 (88c:81137)

121. Goldstein, J. A. and G.R. Rieder (1991) Thomas-Fermi theory with an external magnetic field, J. Math. Phys. 32, pp. 2907-2917. MR1130565|(92k:81238) 
122. Goldstein, J. A., G.R. Goldstein and Wenyiao JiA (1995) Thomas-Fermi theory with magnetic fields and the Fermi-Amaldi correction, Diff. Int. Equ. 8, pp. 1305-1316. MR:1329842 (96g:81288)

123. Gustafson, S.J. and I.M. Sigal (2003) Mathematical concepts of quantum mechanics, Universitext, Springer. MR2002159 (2004g:81002)

124. Hagedorn, G.A. (1996) Crossing the Interface between Chemistry and Mathematics, Notices of the AMS 43, pp. 297-299. MR1375215

125. Hagedorn, G.A. (1980) A time-dependent Born-Oppenheimer approximation, Commun. Math. Phys. 77, pp. 1-19. MR0588684 (82a:81040)

126. Hehre, W.J., L. Radom, P.v.R. Schleyer, and J.A. Pople (1986) Ab initio molecular orbital theory (Wiley).

127. Hoffmann-Ostenhof, M., T. Hoffmann-Ostenhof and T. Sorensen (2001) Electron wavefunctions and densities for atoms, Ann. Henri Poincaré 2, no. 1, pp. 77-100. MR1823834 (2003c:81231)

128. Hunziker, W. and I.M. Sigal (2000) The quantum N-body problem, J. Math. Phys., vol. 41, pp. 3348-3510. MF 1768629 (2001g:81267)

129. Iório, R. J., Jr. and R.D. MARChesin (1984) On the Schrödinger equation with timedependent electric fields, Proc. Royal Soc. Edinburgh 96, pp. 117-134. MR0741652 (85k:35179)

130. JAY, L.O., H. KIM, Y. SAAD and J.R. ChELIKOWSKI (1999) Electronic structure calculations for plane wave codes without diagonalization, Comp. Phys. Comm. 118, pp. 21-30.

131. Jones, R.O. and O. Gunnarsson (1989) The density functional formalism, its applications and prospects, Rev. Mod. Phys. 61, pp. 689-746.

132. Kato, T. (1957) On the eigenfunctions of many-particle systems in quantum mechanics, Comm. Pure Appl. Math. 10, pp. 151-177. MR0088318 (19,501a)

133. Kato, T. (1980) Perturbation theory for linear operators (Springer). MR 1335452 (96a:47025)

134. Kittel, Ch. (1996) Introduction to Solid State Physics, 7th Ed., Wiley.

135. Klahn, B. and W.A. Bingel (1977) The convergence of the Rayleigh-Ritz method in Quantum Chemistry, Theor. Chim. Acta 44, pp. 26-43.

136. Kohn, W. (1999) Nobel Lecture: Electronic structure of matter-wave functions and density functionals, Rev. Mod. Phys. 71, pp. 1253-1266.

137. KouteckÝ, J. and V. Bonacic (1971) On convergence difficulties in the iterative HartreeFock procedure, J. Chem. Phys. 55, pp. 2408-2413.

138. Kudin, K. and G.E. Scuseria (1998) A fast multipole algorithm for the efficient treatment of the Coulomb problem in electronic structure calculations of periodic systems with Gaussian orbitals, Chem. Phys. Lett. 289, pp. 611-616.

139. Kudin, K., G.E. Scuseria and E. CAnCÈs (2002) A black-box self-consistent field convergence algorithm: one step closer, J. Chem. Phys. 116, pp. 8255-8261.

140. LE BRIS, C. (1993) Quelques problèmes mathématiques en chimie quantique moléculaire, Ph.D. thesis, École Polytechnique.

141. Le Bris, C. (2003) Guest Editor, Handbook of Numerical Analysis, Special Volume, Computational Chemistry, volume X, North-Holland. MR.2008385 (2005c:81001)

142. Le BrIs, C. (1993b) Some results on the Thomas-Fermi-Dirac-von Weizsäcker model, Diff. Int. Equ. 6, pp. 337-353. MR1195387 (94a:81147)

143. Le BRIS, C. (1994) A general approach for multiconfiguration methods in quantum molecular chemistry, Ann. Inst. Henri Poincaré Anal. non linéaire 11, pp. 441-484. MR1287241 (95m:81231)

144. LE BRIS, C. (1995) On the spin polarized Thomas-Fermi model with the Fermi-Amaldi correction, Nonlinear Analysis, Theory, Methods and Applications 25, pp. 669-679. MR 1341520 (96k:81298)

145. LE BRIS, C., Y. MADAY and G. TURINici (2004) Towards efficient numerical strategies for quantum control, in Quantum control: Mathematical and numerical challenges, American Mathematical Society, CRM proceedings series. MR2043517(2004j:00023)

146. LE BRIS, C. (2005) Computational chemistry from the perspective of numerical analysis, to appear in Acta Numerica.

147. Lester, W.A., Jr. (1997-2002) Recent advances in quantum Monte Carlo methods, 2 volumes, World Scientific. 
148. Levine, I.N. (1991) Quantum Chemistry (Prentice Hall).

149. Lewin, M. (2002) The multiconfiguration methods in Quantum Chemistry: Palais-Smale condition and existence of minimizers, C. R. Acad. Sc. Paris Ser. I 334, pp. 299-304. MR $1891007(2002 \mathrm{~m}: 81271)$

150. LEWIN, M. (2004) Solutions of the Multiconfiguration Equations in Quantum Chemistry, Arch. Rat. Mech. Anal., vol. 171, 1, pp. 83-114. MR.2029532

151. LieB, E.H. (1984) Bound on the maximum negative ionization of atoms and molecules, Phys. Rev. A 29, pp. 3018-3028.

152. LieB, E.H. (1989) Kinetic energy bounds and their applications to the stability of matter, in Schrödinger operators, H. Holden and A. Jensen, eds., Lecture Notes in Physics, vol. 345, pp. 371-382. MR 1037324 (91a:82045)

153. LieB, E.H. (1990) The stability of matter: from atoms to stars, Bull. A.M.S. 22, pp. 1-49. MR.1014510 (91f:81002)

154. LieB, E.H. (1985) Density functionals for Coulomb systems, in: R.M. Dreizler and J. da Providencia, eds., Density Functional Methods in Physics (Plenum, New York), pp. $31-80$.

155. Lieb, E.H. and B. Simon (1977) The Hartree-Fock theory for Coulomb systems, Comm. Math. Phys. 53, pp. 185-194. MR0452286 (56:10566)

156. Lieb, E.H. and B. Simon (1977) The Thomas-Fermi theory of atoms, molecules and solids, Advances in Mathematics 23, pp. 22-116. MR0428944(55:1964)

157. Lieb, E.H. (1979) A lower bound for Coulomb energies, Physics Lett., vol. 70A, pp. 444-446. MR 0588128 (83g:82008)

158. LieB, E.H. and S. OxFord (1981) Improved lower bound on the indirect Coulomb energy, Int. J. Quant. Chem., vol. 19, pp. 427-439.

159. Lieb, E.H. (1981) Thomas-Fermi and related theories of atoms and molecules, Rev. Mod. Phys. 53, pp. 603-641. MR0629207 (83a:81080a)

160. Lieb, E.H. (1983) Density functionals for Coulomb systems, Int. J. Quantum Chem. 24, pp. 243-277.

161. Lieb, E.H. (1984) Bound of the maximum negative ionization of atoms and molecules, Phys. Rev. A 29, pp. 3018-3028.

162. Lieb, E.H. and W. ThirRing (1976) Inequalities for the moment of the eigenvalues of the Schrödinger hamiltonian and their relation to Sobolev inequalities, in Studies in Mathematical Physics, E.H. Lieb, B. Simon and A. Wightman, eds., Princeton Univ. Press, pp. 269-303.

163. Lieb, E.H. and W. ThirRing (1986) Universal nature of van der Waals forces for Coulomb systems, Phys. Rev. A 34, pp. 40-46.

164. Lions, P.-L. (1985) Hartree-Fock and related equations, in Nonlinear partial differential equations and their applications, Lect. Coll. de France Semin., Vol. IX, Pitman Res. Notes Math. Ser. 181, pp. 304-333. MR0992653 (90i:35251)

165. Lions, P.-L. and A. MAJdA (2000) Equilibrium statistical theory for nearly parallel vortex filaments, Commun. Pure Appl. Math. 53, no. 1, pp. 76-142. MR1715529 (2000h:76086)

166. Lions, P.-L. (1987) Solutions of Hartree-Fock equations for Coulomb systems, Comm. Math. Phys. 109, pp. 33-97. MR0879032 (88e:35170)

167. Lions, P.-L. (1985) The concentration-compactness principle in the calculus of variations. The limit case. I, II, Rev. Mat. Iberoam. 1, No. 1, pp. 145-201 and No. 2, pp. 45-121. MR0834360 (87c:49007) MR0850686 (87j:49012)

168. Lions, P.-L. (1984) The concentration-compactness principle in the calculus of variations. The locally compact case. I and II, Ann. Inst. Henri Poincaré, Anal. Non Linéaire 1, pp. 109-145 and pp. 223-283. MR.0778970(87e:49035a), MR.0778974 (87e:49035b)

169. Lions, P.-L. (1996) Remarks on mathematical modelling in quantum chemistry, Computational Methods in Applied Sciences, Wiley, pp. 22-23.

170. Liv, W.K. et al., eds. (2004) Special issue on multiple scale methods for nanoscale mechanics and materials, Comp. Math. Appl. Mech. Eng. 193, vols. 17-20. MR2069426

171. MCWeEny, R. (1992) Methods of molecular quantum mechanics, 2nd edition (Academic Press).

172. Maday, Y. and G. TURINICI (2003) Error bars and quadratically convergent methods for the numerical simulation of the Hartree-Fock equations, Numer. Math. 94, no. 4, pp. 739-770. MR:1990591|(2005c:81050) 
173. MARCH, N.H. (1992) Electron density theory of atoms and molecules (Academic Press).

174. Nijboer, B.R.A, and W.J. Ventevogel (1979) On the configuration of systems of interacting particles with minimum potential energy per particle, Physica, vol. 98A, pp. 274-288. MR $0546896[(80 \mathrm{i}: 81080)$

175. NijBoer, B.R.A, and W.J. Ventevogel (1979) On the configuration of systems of interacting particles with minimum potential energy per particle, Physica, vol. 99A, pp. 569-580. MR $0552855[(83 \mathrm{e}: 81102)$

176. Ordejon, P., D.A. Drabold and R.M. Martin (1995) Linear system-size scaling methods for electronic structure calculations, Phys. Rev. B 51, pp. 1456-1476.

177. Pagano, S. and R. Paroni (2003) A simple model for phase transitions: from the discrete to the continuum problem, Quart. Appl. Math. 61, no. 1, pp. 89-109. MR.1955225(2003k:74053)

178. PARR, R. G. and W. YANG (1989) Density functional theory of atoms and molecules (Oxford University Press).

179. Paturel, E. (2000) Solutions of the Dirac-Fock equations without projector, Ann. Henri Poincaré 1, no. 6, pp. 1123-1157. MR.1809795 (2001k:81369)

180. Payne, P. W. (1979) Density functionals in unrestricted Hartree-Fock theory, J. Chem. Phys. 71, pp. 490-496.

181. PISANI, C., ed. (1996) Quantum mechanical ab initio calculation of the properties of crystalline materials, Lecture Notes in Chemistry 67, Springer.

182. Pulay, P. (1982) Improved SCF convergence acceleration, J. Comp. Chem. 3, pp. 556-560.

183. RAABE, D. (1998) Computational materials science, Wiley.

184. Radin, C. (1981) Ground states for soft disks, J. Stat. Phys., vol. 26, pp. 365-373. MR 0643714 (83b:82011)

185. REED, M. and B. SIMON (1975-1980) Methods of modern mathematical physics, in 4 volumes, Academic Press. MR.0493419 (58:12429a), MR0493420 (58:12429b), MR0529429 $(80 \mathrm{~m}: 81085)$ MR0493421 (58:12429c)

186. Saunders, V. R. and I.H. Hillier (1973) A "level-shifting" method for converging closed shell Hartree-Fock wavefunctions, Int. J. Quantum Chem. 7, pp. 699-705.

187. Schechter, M. (1981) Operator methods in quantum mechanics (North Holland). MR 0597895 (83b:81004)

188. SCUSERIA, G.E. (1999) Linear scaling density functional calculations with Gaussian orbitals, J. Phys. Chem. A 103, pp. 4782-4790.

189. SoloveJ, J.-P. (1990) Universality in the Thomas-Fermi-von Weizsäcker model of atoms and molecules, Comm. Math. Phys. 129, pp. 561-598. MR1051505 (91b:81191)

190. SoloveJ, J.-P. (1991) Proof of the ionization conjecture in a reduced Hartree-Fock model, Invent. Math. 104, pp. 291-311. MR1098611 (92f:81238)

191. SoloveJ, J.-P. (1996) The size of atoms in Hartree-Fock theory, Lars Hörmander et al. (eds.), Partial differential equations and mathematical physics, The Danish-Swedish analysis seminar, 1995, Proceedings, Birkhäuser, Prog. Nonlinear Differ. Equ. Appl. 21, pp. 321-332. MR:1380999 (96k:81292)

192. SpruCH, L. (1991) Pedagogic notes on Thomas-Fermi theory (and on some improvements): atoms, stars and the stability of bulk matter, Rev. Mod. Phys. 63, pp. 151-209.

193. Stanton, R.E. (1981) The existence and cure of intrinsic divergence in closed shell SCF calculations, J. Chem. Phys. 75, pp. 3426-3432.

194. Stanton, R.E. (1981) Intrinsic convergence in closed-shell SCF calculations. A general criterion, J. Chem. Phys. 75, pp. 5416-5422.

195. StaRikov, E.B. (1993) On the convergence of the Hartree-Fock self-consistency procedure, Mol. Phys. 78, pp. 285-305. MR1203047 (94a:81149)

196. Struwe, M. (1990) Variational methods. Applications to nonlinear partial differential equations and Hamiltonian systems (Springer). MR.1078018 (92b:49002)

197. Swirless, B. (1935) The relativistic self-consistent field, Proc. Roy. Soc. A 152, pp. 625-649.

198. Swirless, B. (1936) The relativistic interaction of two electrons in the self-consistent field method, Proc. Roy. Soc. A 157, pp. 680-696.

199. Szabo, A. and N.S. Ostlund (1982) Modern quantum chemistry: an introduction to advanced electronic structure theory (MacMillan).

200. Talman, J.D. (1986) Minimax principle for the Dirac equation, Phys. Rev. Lett. 57, 9, pp. 1091-1094. MR0854208 (87i:81039) 
201. Thirring, W. (1983) A Course in Mathematical Physics, in 4 volumes (Springer). MR0507189 (80d:70003) MR0553112 (80e:78001b), MR0625662 (84m:81006) MR0681697 $(84 \mathrm{~m}: 82004 \mathrm{~b})$

202. Turinici, G., H. Rabitz and E. Brown (2003) Control of Quantum Dynamics: Concepts, Procedures and Future Prospects in Handbook of Numerical Analysis, Special Volume, Computational Chemistry, volume X, North-Holland. MR2008399

203. Ventevogel, W.J. (1978) On the configuration of a one-dimensional system of interacting particles with minimum potential energy per particle, Physica, vol. 92A, p. 343.

204. Yserentant, H. (2004) On the electronic Schrödinger equation, preprint.

205. Yserentant, H. (2004) On the regularity of the electronic Schrödinger equation in Hilbert spaces of mixed derivatives, Numer. Math. 98, pp. 731-759. MR2099319

206. Yserentant, H. (2004) Sparse grid spaces for the numerical solution of the electronic Schrödinger equation, Numer. Math., submitted.

207. Zhislin, G.M. (1960) Discussion of the spectrum of Schrödinger operators for systems of many particles (Russian), Tr. Moskov. Mat. Obshch. 9, pp. 81-120.

Cermics, École Nationale des Ponts et Chaussées, 6 \& 8, avenue Blaise Pascal, 77455 Marne-La-Vallée, France

E-mail address: lebris@cermics.enpc.fr

Collège de France, 11, place Marcelin Berthelot, 75231 Paris Cedex 05, France

E-mail address: lions@dmi.ens.fr 